\title{
EFFECTS OF LOW INTENSITY \\ PULSED ULTRASOUND AND LOW LEVEL HEAT ON BONE CELLS
}

\author{
By \\ Judith Weidman \\ B.Eng. Ryerson University, 2002 \\ Toronto, Ontario
}

\begin{abstract}
A thesis
presented to Ryerson University

in partial fulfillment of the

requirements for the degree of
\end{abstract}

Master of Science

in the Program of

Biomedical Physics

Toronto, Ontario, Canada, 2010

(C) Judith Weidman, 2010 


\section{Author's Declaration}

I hereby declare that I am the sole author of this thesis.

I authorize Ryerson University to lend this thesis to other institutions or individuals for the purpose of scholarly research.

Judith Weidman

I further authorize Ryerson University to reproduce this thesis by photocopying or by other means, in total or in part, at the request of other institutions or individuals for the purpose of scholarly research.

Judith Weidman 


\section{Abstract}

\section{Judith Weidman. “Effects of Low Intensity Pulsed Ultrasound and Low Level Heat on Bone Cells”, M. Sc. Biomedical Physics, Ryerson University, Toronto, 2010.}

Both Low Intensity Pulsed UltraSound (LIPUS) and low level heat have separately been shown to improve mineralization in bone cell cultures (Unsworth et al 2007, Leon et al 1993). This study examines the effect of concurrent LIPUS and low level heat on MC3T3-E1 bone cell cultures. The treatment groups were: LIPUS, heat, LIPUS + heat, and control. The LIPUS intensity was $\mathrm{I}_{\text {SATA }}=10 \mathrm{~mW} / \mathrm{cm}^{2}$ at $f=1.5 \mathrm{MHz}$, and heat was applied at $40^{\circ} \mathrm{C}$ for 40 minutes each day over 15 days. The LIPUS + heat group received the treatments concurrently.

The groups were compared using Alizarin Red staining to measure the degree of cell mineralization. All treatment groups showed statistically significantly improved mineralization over the control; however, there was no statistical difference between the LIPUS and the LIPUS + heat groups. Early results suggest that concurrent heat and LIPUS on MC3T3-E1 bone cells has no additive effect on mineralization. 


\section{Acknowledgements}

I would like to thank my supervisors Dr. Jahan Tavakkoli, and Dr. Howard Ginsberg for their support during this research project. Dr. Tavakkoli’s intelligence, inquisitiveness and diligence helped me through my thesis. Dr. Ginsberg's calm energy and positive focus that reminded the results would come with time. It has been a privileged to have worked with such knowledgeable professional scientists.

I would like to thank Smith \& Nephew Inc., Memphis, TN, for providing technical advice and a research device.

I would also like to thank my supervisory committee, Dr. Michael Kolios, and Dr. Ana Pejovic-Milic for their valuable guidance throughout this project, and Dr. Min Rui and Arthur Worthington for technical assistance throughout my experiments. This work would not have been possible without their support.

I would like to thank my colleagues in the Biomedical Physics Master's program at Ryerson University who have made my experience here fun and memorable.

Most of all I would like to thank my family who encouraged me to keep taking on challenges.

This work was supported by the Ryerson University Dean’s Start-up Fund that was made available to Dr. Tavakkoli. 


\section{Table of Contents}

AUTHOR'S DECLARATION ......................................................................... II

ABSTRACT ................................................................................................................. III

ACKNOWLEDGEMENTS ........................................................................ IV

TABLE OF CONTENTS ...................................................................................

LIST OF FIGURES ............................................................................... VIII

LIST OF TABLES .....................................................................................XIII

CHAPTER 1 INTRODUCTION AND BACKGROUND................................1

1.1 The Physics of Ultrasound (Cobbold 2007) ……………........................................................ 1

1.2 Ultrasound and Bone ........................................................................................................ 9

1.2.1 The History of Ultrasound in Bone Healing ............................................................... 9

1.2.2 Low Intensity Pulsed Ultrasound (LIPUS) .............................................................. 11

1.2.3 LIPUS Mechanisms of Action in Bone Fracture Healing ......................................... 14

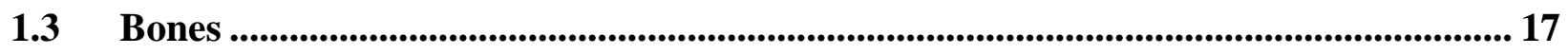

1.3.1 Bone function (Bartel 2006) …………………….............................................. 17

1.3.2 MC3T3-E1 bone cells........................................................................................... 21

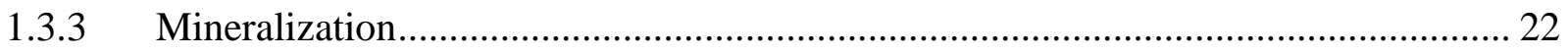

1.4 Heating ................................................................................................................... 22

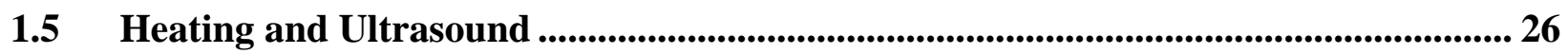

1.6 Physics of Light Absorbance: Beer's Law (Workman 1998)......................................... 27

$1.7 \quad$ Alizarin Red (Puchtler et al 1969) ........................................................................................ 28

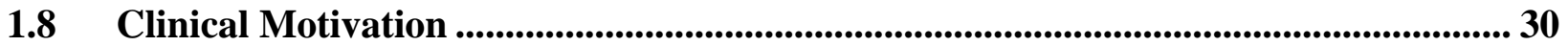

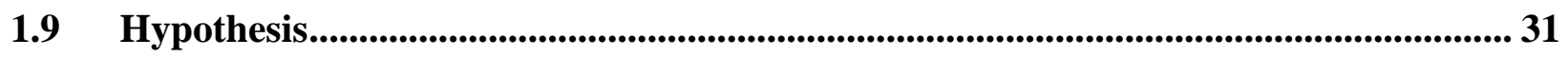

1.9.1 Specific Aims .............................................................................................. 31

CHAPTER 2 EXOGEN® LIPUS DEVICE AND ITS ACOUSTIC

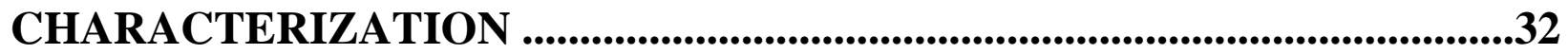

2.1 Introduction................................................................................................................................... 32 


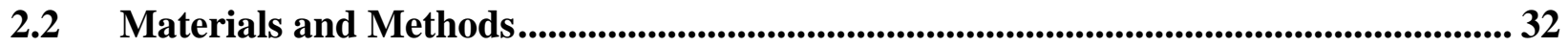

2.2.1 Exogen ${ }^{\circledR}$ LIPUS Device ……………………................................................. 32

2.2.2 Acoustic Output Measurement System .................................................................... 34

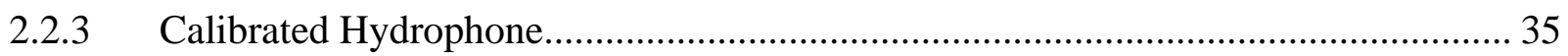

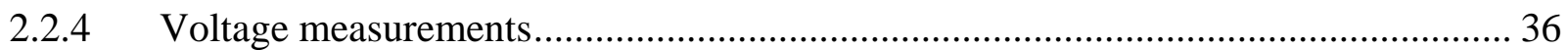

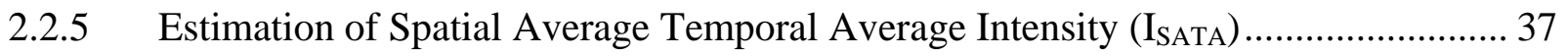

2.2.6 Estimation of Frequency, Pulse Duration, Pulse repetition, Duty Cycle ................... 38

2.2.7 Effect of polystyrene cell culture plate on acoustic intensity .................................... 38

2.2.8 Calculation of Intensity Lose due to Polystyrene Cell Plate ........................................ 39

2.2.9 Exogen ${ }^{\circledR}$ Device Acoustic Beam Simulation ......................................................... 42

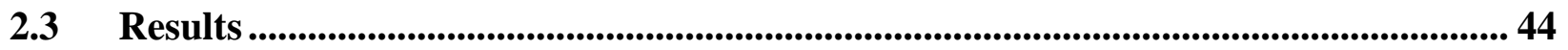

2.3.1 Estimation of Acoustic Intensity ........................................................................... 44

2.3.2 Effective Radiating Area ....................................................................................... 46

2.3.3 Estimation of Pulse Frequency, Pulse Duration, Pulse Repetition Frequency (PRF), and Duty Cycle (DC) ............................................................................................................ 47

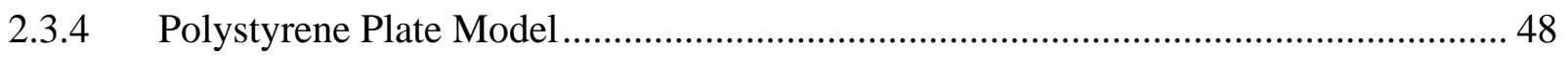

2.3.5 Computer Simulations Results - Intensity Simulation Results .................................. 49

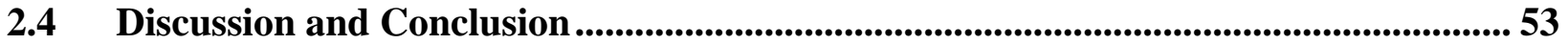

CHAPTER 3 CELL CULTURE EXPERIMENTS: MATERIALS AND

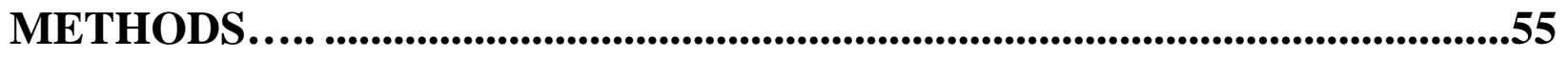

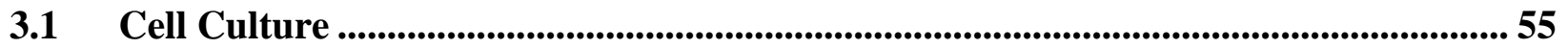

3.2 Cell Culture Equipment ..................................................................................................... 55

$3.3 \quad$ Preparation of Work Place ................................................................................................ 56

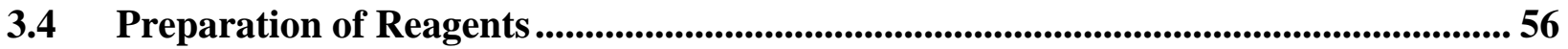

3.4.1 aMEM for Cell Culture ...................................................................................... 56

3.4.2 Ascorbic Acid $(50 \mu \mathrm{g} / \mathrm{ml})$ - Cell Differentiation Supplement...................................... 56

3.4.3 $\quad \beta$-Glycerol Phosphate (3mM) - Cell Differentiation Supplement ............................... 57

3.4.4 Differentiation Supplement for aMEM Media.......................................................... 57

3.4.5 Alizarin Red for Cell Culture Staining ................................................................ 58

3.4.6 Perchloric Acid for Alizarin Red Stain Rehydration................................................... 58

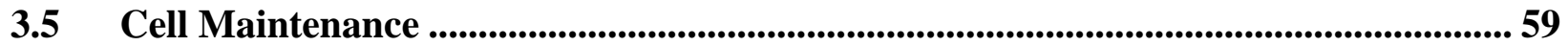


3.5.1 Splitting and Passaging Cells ............................................................................... 59

3.5.2 Digesting and Re-Suspending Cells ................................................................... 59

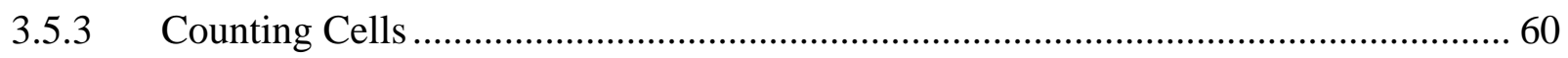

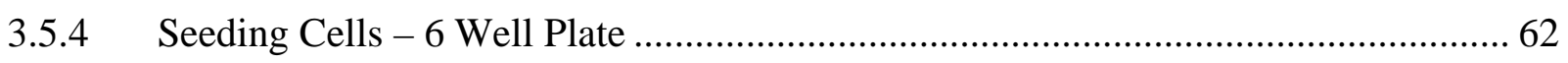

3.6 Fixing cell culture and Staining for mineralization ............................................................ 62

3.7 De-Staining and Quantification .......................................................................................... 63

3.8 Cell Treatment Experimental Protocol.............................................................................6 64

3.8.1 Cell Culture - Pre Experiment Testing....................................................................... 66

3.8.2 LIPUS Treatment Effect - Comparison to Published Results ....................................... 69

3.8.3 Test of Hypothesis - Combined Treatment effect....................................................... 69

3.9 Spectral Measurements of Alizarin Red........................................................................ 70

3.10 Statistical Study Design (Blair and Taylor 2008)............................................................... 71

3.10.1 Comparison of Ultrasound and Heat to Control ........................................................ 71

3.10.2 Comparison of Ultrasound + Heat to Ultrasound ..................................................... 73

CHAPTER 4 CELL CULTURE EXPERIMENTS: RESULTS .....................74

4.1 Differentiation ............................................................................................................................. 74

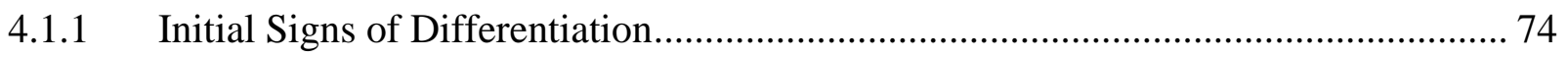

4.1.2 Later signs of Differentiation - Calcification and Improved Mineralization.............. 77

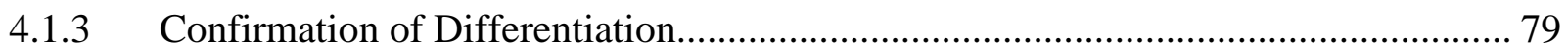

4.2 Ultrasound Treatment Effect - Comparison to Published Results.................................. 84

4.3 Results - Test of Hypothesis - Combined Treatment effect............................................... 89

4.4 Spectrum measurements of Alizarin Red ......................................................................... 95

CHAPTER 5 DISCUSSION AND CONCLUSION .......................................99

5.1 Discussion.................................................................................................................................... 99

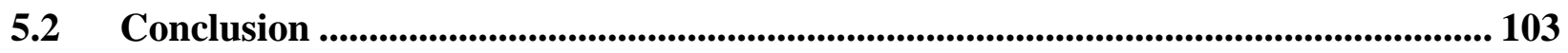

5.3 Future work............................................................................................................................... 104

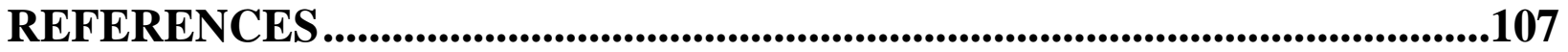




\section{List of Figures}

Figure 1-1: Ultrasound transducer schematic (Zagzebski 1996)................................................... 2

Figure 1-2: Longitudinal and Transverse Waves (Cobbold 2007)................................................ 3

Figure 1-3: Absorption and attenuation of ultrasound. (a) Distinguishing between the absorbed, scattered and attenuated waves for a simple plane specimen . (b) Change in intensity for the passage of a plane wave through an incremental distance dx. (Cobbold 2007), .......... 4

Figure 1-4: Piston Transducer (Zemanek 1971) .................................................................. 7

Figure 1-5: Schematic geometry of an ultrasound beam at the muscle/bone interface. Ultrasound propagates through the muscle and then impinges against the muscle/bone interface at a distance of $\mathrm{Zb}$. The cortical layer is from $\mathrm{Zb}$ to $\mathrm{Zc}$ and the spongy/marrow region from Zc to Ze. $\alpha, \beta$, and $\gamma$ are the angles of the incident longitudinal wave (Il), the refracted longitudinal wave (Tl), and the refracted shear wave (Ts), respectively (Liauh et al 2004).

Figure 1-6: A) Control limb - no union has formed B) Ultrasound treated limb - significant callus formation (Duarte 1983).

Figure 1-7: The effect of LIPUS treatment on calcium nodule formation of human periosteal cells after 4 weeks of treatment. Image analysis shows the difference in color intensity between the treatment and control group. The asterisk indicates there was a statistically significant difference $(\mathrm{p}<0.001)$. (Error bar $=1$ standard deviation.) (Leung et al 2004a).

Figure 1-8: Alizarin red staining in cultures of MC3T3-E1. Significant differences were observed at days 10, 20 and 25. Asterisks represent a significant increase ( $\left.\mathrm{p} \_0.005\right)$ vs. control cultures. Results are expressed as mean of six replicates error bar $=$ standard

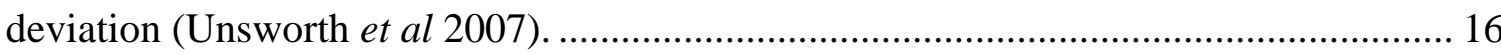

Figure 1-9: Hierarchical structures in bone: (a) Femur (b) Osteons ( $\sim 100 \mu \mathrm{m})$ (c) Collagen fiber ( $\sim 5 \mu \mathrm{m}$ in diameter) consisting of bundles of collagen fibrils ( $\sim 500 \mathrm{~nm}$ in diameter each). (d) Striped collagen fibril consisting of a staggered arrangement of collagen 
molecules ( $\sim 1.5 \mathrm{~nm}$ in diameter, $300 \mathrm{~nm}$ long) with embedded minerals $(\sim 2-20 \mathrm{~nm}$ lat.,15 nm wide, $30 \mathrm{~nm}$ long.). (e) Collagen molecule triple helix (Buger 2008)............. 18

Figure 1-10: Mineral deposits in a primary MC3T3-E1 cultures after 24 days in culture mineralized matrix vesicles among collagen fibrils (Sudo et al 1983) 19

Figure 1-11: Histological analysis of fracture healing. The progression of repair on days $1,3,7,14,21$, and 28. On day 7, extensive soft callus is seen forming around the injured bone. At day 14, the soft callus becomes mineralized to form new bone and achieve union by day 21 and 28 (x40) (Stroncek and Reichert 2008).

Figure 1-12: Time dependant differentiation of MC3T3 cells. Yellow indicates phenotype changes as the cell differentiates. Blue-Gray indicates protein expressions as the cells differentiates into a mineralized osteoblast (Beck Jr. et al 2000)

Figure 1-13: A) The arrow indicated that the heat treated bone has significantly thicker bone callus than B) the control bone. C) The arrow indicates that the histological sample show heat treated bone has significantly improved mineral deposits. D) The arrow indicates that the control sample shows an open structure (Leon et al 1993)................................. 23

Figure 1-14: Effect of hyperthermia on MC3T3-E1 cell viability. The bars represent the number of days in culture at $40^{\circ} \mathrm{C}$. Cell viability is expressed as a percentage of control cells cultured at $37^{\circ} \mathrm{C} . *: \mathrm{P}<0.05, * * \mathrm{P}<0.01, * * *: \mathrm{P}<0.001$ (Flour et al 1992).

Figure 1-15: Effect of hyperthermia on MC3T3-E1 proliferation. at $37^{\circ} \mathrm{C}(\square)$ and $40^{\circ} \mathrm{C}(\boldsymbol{\Delta})$. Each value is the mean of three determinations. ${ }^{*}: \mathrm{P}<0.05,{ }^{*} \mathrm{P}<0.01$, ${ }^{* * *}: \mathrm{P}<0.001$ (Flour

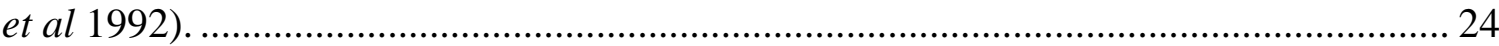

Figure 1-16: Calcium deposition by BMSCs after multiple 1-h exposure to different temperatures. The cells were exposed to different temperatures for $1 \mathrm{~h}$ at intervals of every 3 days. After 21 days the cultures were stopped $*: P<0.05$, compared with control $\left(37^{\circ} \mathrm{C}\right)$ (Shui and Scutt 2001).

Figure 1-17: Calcium deposition by BMSCs after long-term exposure to low and high temperatures. After incubation at 33,37 , or $39^{\circ} \mathrm{C}$, respectively, for 10 days, the cultures were stopped. *:P $<0.05$, compared with control $\left(37^{\circ} \mathrm{C}\right.$ ) (Shui and Scutt 2001)............ 25

Figure 1-18: Light absorption through a cuvette. 27 
Figure 1-19: Spectrometer measurements using Beer's Law. The absorption of sample A is higher than the absorption of sample B because the concentration of sample A is greater. 28

Figure 1-20: Formation of the calcium alizarin red bond (Puchtler et al 1969).......................... 29

Figure 1-21: Formulation of the alizarin red-S molecule (U.S. National Library of Medicine ). 29 Figure 1-22: Musculoskeletal impairments for 1995 (Bone and Joint Decade 2009)................. 30

Figure 2-1: A) Exogen ${ }^{\circledR} 4000$ + bone healing clinical system ${ }^{\circledR}$ and B) Exogen ${ }^{\circledR}$ multi-transducer research device with an array of 6 transducers. .............................................................. 33

Figure 2-2: Acoustic output measurement set up using the calibrated hydrophone..................... 34

Figure 2-3: The calibrated hydrophone sensitivity table (Precision Acoustics 2007)................... 36

Figure 2-4: Cut off intensity for calculating active intensity and the effective radiating area

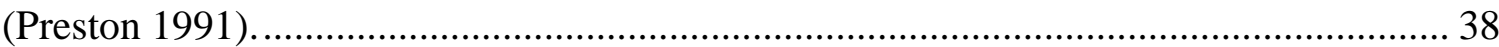

Figure 2-5: Schematic of intensity loss of at the water polystyrene interface. $\mathrm{I}_{\mathrm{f}}$ indicates intensity remaining after attenuation, while $\mathrm{I}_{\mathrm{r}}$ indicates loss due to reflection. 39

Figure 2-6: Frequency dependant attenuation of ultrasound through polystyrene (Waters et al 2003).

Figure 2-7: Coordinate system and geometry used to define pressure at any point in the

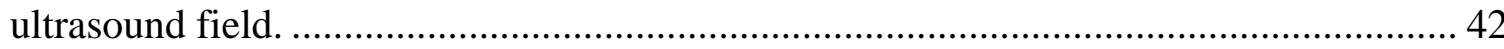

Figure 2-8: Measured Lateral Intensity Profile............................................................................... 45

Figure 2-9: Measured Axial Intensity Profile....................................................................... 45

Figure 2-10: Lateral Intensity (log scale)............................................................................... 46

Figure 2-11: Hydrophone signal at 13mm away from transducer face. ...................................... 47

Figure 2-12: Intensity comparison with and without polystyrene cell plate. For measured intensities with and without the polystyrene plate, the plate was placed against the

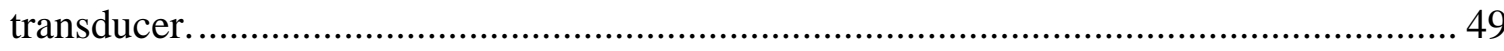

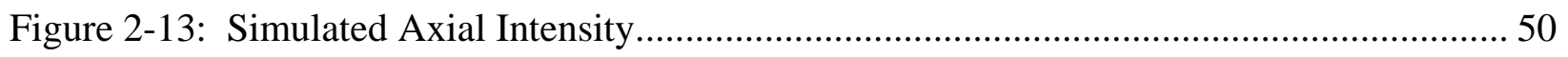

Figure 2-14: Simulated vs. Measured Axial intensity 11-20 mm................................................. 51

Figure 2-15: Lateral intensity profiles where the intensity is averaged over $1 \mathrm{~mm}$ diameter thick rings A) $11 \mathrm{~mm}$, B) $12 \mathrm{~mm}$, C) $13 \mathrm{~mm}$, D) $15 \mathrm{~mm}$................................................ 52

Figure 2-16: Simulated Lateral Intensity vs. Measured Intensities. ................................................ 53

Figure 3-1: Hemocytometer grid (Maes 2004)..................................................................... 61 
Figure 3-2 Sample selection for spectrometer measurements. Remove the remove the solution from the 6 well plate after 23 hours incubation at room temperature............................ 64

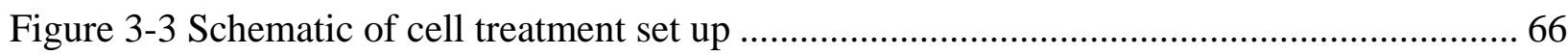

Figure 4-1: Primary MC3T3-E1 cells A) 2 days after seeding B) 6 days after seeding. Images from (Sudo et al 1983). Immortalized MC3T3-E1 from ATCC C) 2 days after seeding

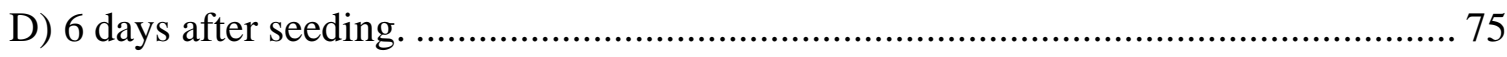

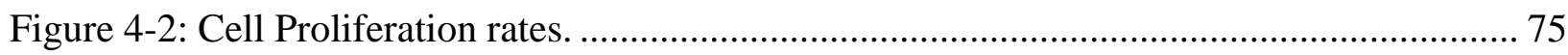

Figure 4-3: Day 15, MC3T3-E1 cell cultures A) Cell culture with differentiation supplement added. B) Cell culture without cell differentiation supplement C) Intact collagen matrix of differentiation supplement positive cell culture. 76

Figure 4-4: MC3T3-E1 alizarin red stained cell cultures on Day 15. Mineralization is dependent on the supplemented concentration of ascorbic acid and $\beta$-glycerol phosphate. 78

Figure 4-5: MC3T3-E1 alizarin red stained cell cultures on Day 15 at $10 \mathrm{X}$ magnification. The dark staining, for $50 \mu$ g ascorbic acid with 3 and $4 \mathrm{nM}$ of $\beta$-glycerol phosphate, indicates calcium deposits. Bar $=200 \mu \mathrm{m}$. 78

Figure 4-6: Spectrometer measurements of rehydrated alizarin red samples. Absorbance measurements indicated that mineralization is dependent on the supplemented concentration of ascorbic acid and $\beta$-glycerol phosphate. 79

Figure 4-7: Differentiation supplement positive and differentiation supplement negative cell cultures over time. (+) indicates that differentiation supplements have been added, (-) indicates that differentiation supplements have not been added. Note: The treated culture was contaminated on Day 10.

Figure 4-8: Optical density of calcium absorption for differentiation supplement positive cell cultures. The treated culture was contaminated on Day 10. The error bar represents standard error of 3 samples.

Figure 4-9: Optical density of calcium absorption for differentiation supplement negative cell cultures. The treated culture was contaminated on Day 10. The error bar represents standard error of 3 samples.

Figure 4-10: Cell culture differentiation shown positive staining for calcification. A) DS+ cell culture - the alizarin red has bonded with the calcium expressed by the cells. B) 
Previously published results show alizarin red has bonded with calcium expressed by the cells (Tang et al 2004).

Figure 4-11: Comparison of US20 treatment to Control treatment for differentiation supplement negative samples. Error bar indicated the standard error of 12 measurements. 85

Figure 4-12: Comparison of US20 treatment to Control treatment for differentiation supplement positive samples. Error bar indicated the standard error of 12 measurements. 86

Figure 4-13: Ultrasound 20 minute treated cell cultures over 15 days. Positions 1-4 are DS+ and Positions 5-6 are DS- for all well plates. 87

Figure 4-14: Control treated cell cultures over 15 days. Positions 1-4 are DS+ and Positions 5-6 are DS- for all well plates. 88

Figure 4-15: Comparison of treatment groups by treatment day. All samples are differentiation supplement positive. Error bar indicates a standard error of 18 measurements. 90

Figure 4-16: Comparison of treatments over time. Error bar indicates a standard error of 18 measurements. 91

Figure 4-17: All treatment groups day 5. No apparent mineralization. 92

Figure 4-18: All treatment groups day 10. Mineralization apparent only on 1 well for US40 and 1 for US40 + H40 apparent mineralization. 93

Figure 4-19: All treatment groups day 15. Mineralization apparent on several wells for US40 and US40 + H40 apparent mineralization.

Figure 4-20: Effect of heat shock on mineralized matrix formation for human mesenchymal stem cells. Light grey indicates differentiation supplement positive, dark grey indicates differentiation supplement negative. The supplement to induce differentiation includes vitamin D, $\beta$-glycerol phosphate and ascorbic acid (Nørgaard et al 2006). 95

Figure 4-21: 0.5mM Alizarin Red at pH 3.90. The peak occurs at 420 nm. (Csányi 1980)........ 96

Figure 4-22: Comparison of spectral curves with and without formalin 97

Figure 4-23: Comparison of spectral curves from $315-500 \mathrm{~nm}$. The shape of the spectral curves is similar. 97

Figure 4-24: Spectrum analysis in range of $405 \mathrm{~nm}$. 98 


\section{List of Tables}

Table 2-1: Comparison of Exogen ${ }^{\circledR}$ Device Manufacturer and Measured Characteristics. ....... 48 Table 4-1: Cell Differentiation Statistics - P values. The P value represents the probability that the mean mineralization of the differentiation positive supplements is greater than the differentiation negative supplement within each treatment. Statistical significance was reached by day 5. P values less than 0.05 are considered statistically significant. (*)The treated culture was contaminated on Day 10.

Table 4-2: Treatment Effect Statistics - P values. The $\mathrm{P}$ value represents probability that the mean mineralization of the Treatment DS+ treatments is greater than the Control DS+ treatment. P values less than 0.05 are considered statistically significant. (*)The treated culture was contaminated on Day 10.

Table 4-3: Cell Culture Differentiation Statistics - P values. The P value represents the probability that the mean mineralization of DS+ cell culture is greater than the DS- cell culture in the same treatment group. Statistical difference reached by day 10. P values less than 0.05 are considered statistically significant.

Table 4-4: Treatment Effect Statistics - P values. The P value represents the probability that the mean mineralization of the treatment is greater than that of the control. Statistical difference reached by day 10 . P values less than 0.05 are considered statistically significant

Table 4-5: Mean optical absorbance of treatment samples.

Table 4-6: Treatment Effect Statistics - P values. The P value represents the probability that the mean mineralization of the treatment is greater than that of the control. All day 5 measurements are statistically significantly greater than day $0(\mathrm{P}=0.0001) . \mathrm{P}<0.05$ is statistically significant. 


\section{Chapter 1 Introduction and Background}

\subsection{The Physics of Ultrasound (Cobbold 2007)}

The physics of ultrasound is based on the theory of acoustics. Sound waves were first described by Sir Isaac Newton in the 17th century and described in his paper Principia where sound is described as being pressure moving particles in a fluid (Newton 1999). In 1877, Rayleigh began the first discussions of what has become the basis of modern day acoustics. In the late nineteenth century the Curie brothers discovered the piezoelectric properties of crystals, where an electric current could be generated by applying pressure at certain points on a crystal (Schortinghuis et al 2003). Likewise applying an electric current to a crystal would cause it to deform.

A simple ultrasound system is made up of a piezoelectric crystal attached to a power source (Figure 1-1). The power source drives an alternating current at a resonant frequency through the piezoelectric crystal. With the piezoelectric effect, the crystal displaces, causing a high frequency sound wave to propagate (Schortinghuis et al 2003). The complete system of converting electrical energy into an acoustic wave is called a transducer. When the transducer is coupled to a human body the acoustic wave will transmit through the skin into the body. 


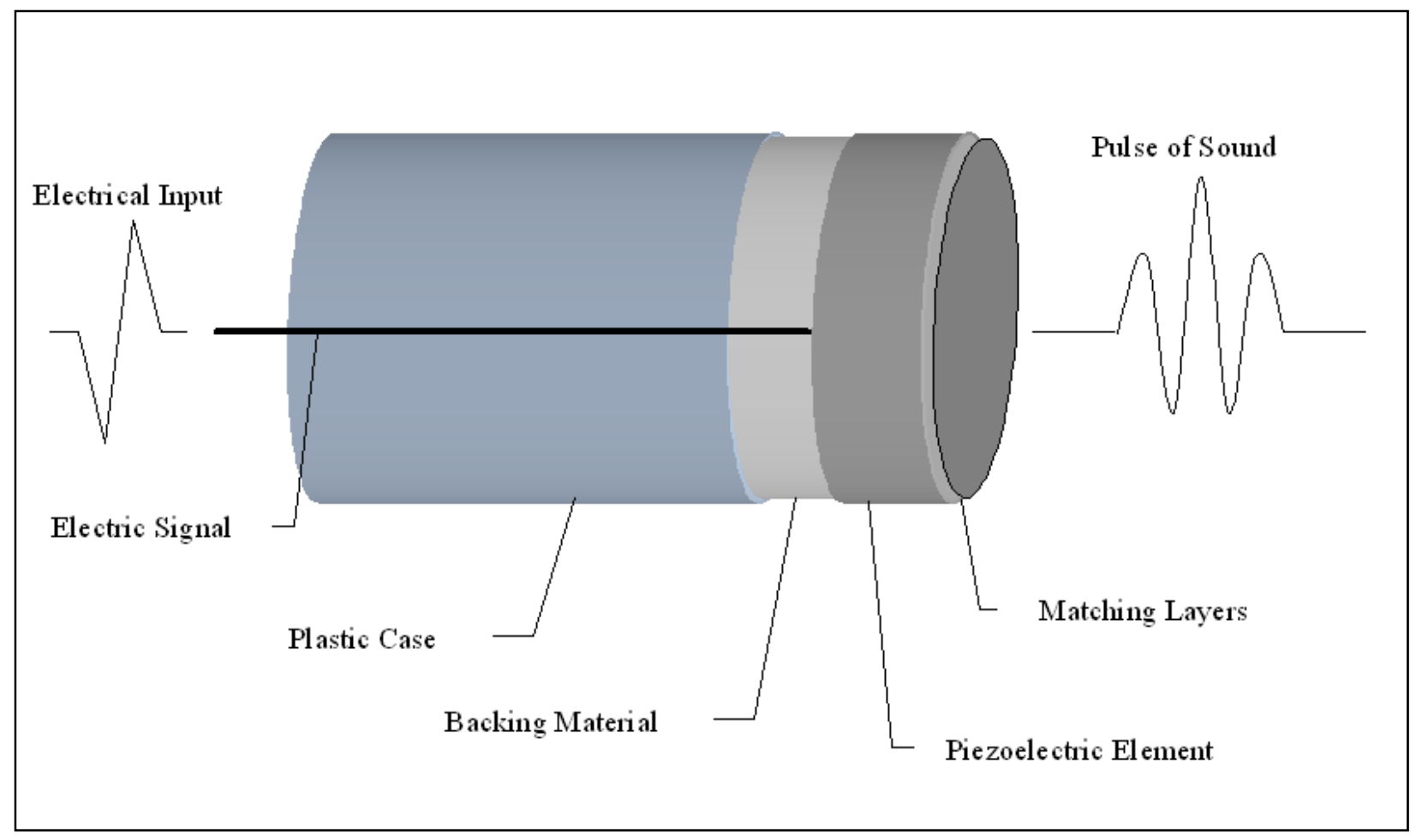

Figure 1-1: Ultrasound transducer schematic (Zagzebski 1996).

Sound waves are the displacement of acoustic particles due to local changes in pressure where mechanical energy is transported through the media. Ultrasound is considered to be a sound wave with frequencies above $20 \mathrm{kHz}$ (the hearing range is between $20 \mathrm{~Hz}$ and $20 \mathrm{kHz}$ ). An acoustic particle is a volume defined in the media such that it is much smaller than the incident wavelength and much larger than inter-atomic spacing (for frequencies less than $1 \mathrm{GHz}$ ). Each cube in Figure 1-2 can be conceived as an acoustic particle. Within the volume of one element the properties of the element are assumed to remain stable. Each volume element within the media can be thought of as connected to the next with a spring and therefore the displacement of one element will displace the others. Through these movements, the energy of a mechanical wave can be transported through the media. 
Waves can move through the media either as a longitudinal wave or as a transverse or shear wave. As illustrated in Figure 1-2 A, when the wave travels parallel to the direction of particle motion the wave is longitudinal. As illustrated in Figure 1-2 B, when it travels perpendicular to the particle motion the wave is a shear wave. In solids both longitudinal and transverse waves propagate through the media, while in liquids predominantly longitudinal waves propagate.

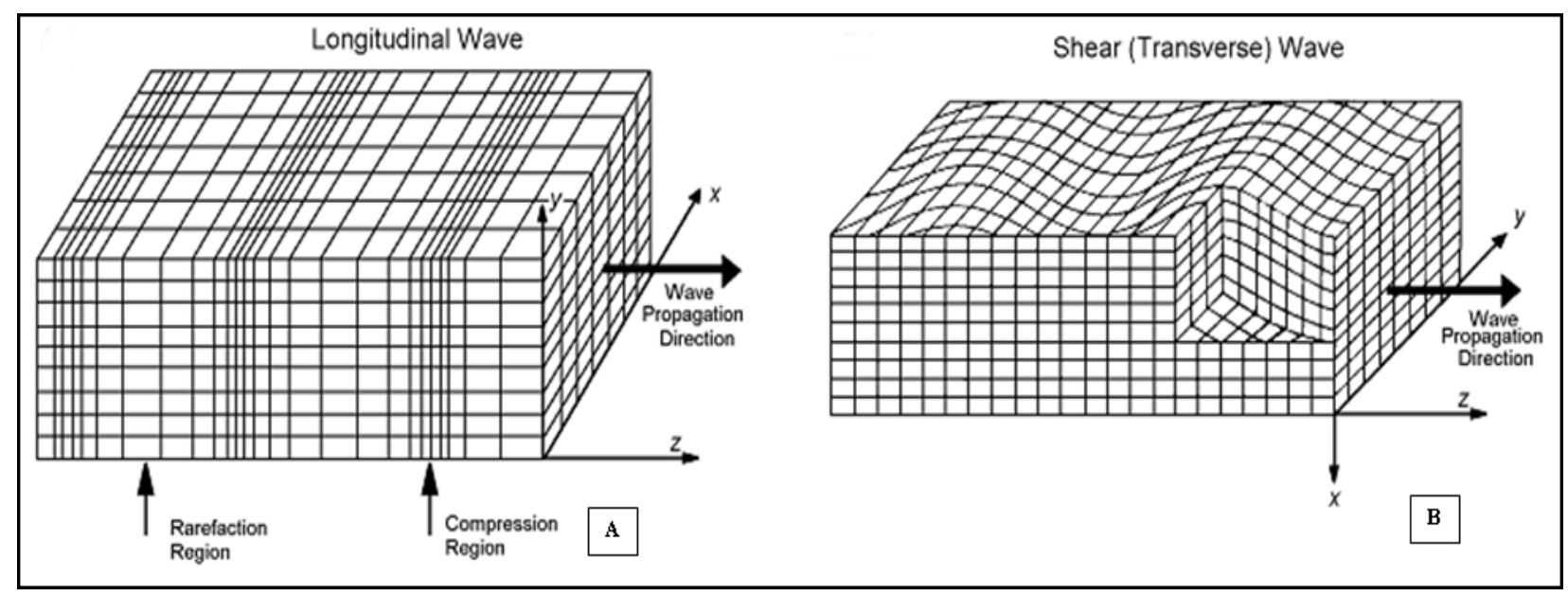

Figure 1-2: Longitudinal and Transverse Waves (Cobbold 2007).

The speed of ultrasound wave traveling through a media is dependent on the properties of the material. The ultrasound wave speed is given by:

$c=\sqrt{\frac{B}{\rho}}$

where $B$ is a property of the medium called bulk modulus or stiffness $\left[\frac{N}{m^{2}}\right]$, and $\rho$ is the density $\left[\frac{\mathrm{Kg}}{\mathrm{m}^{3}}\right]$. The wavelength of sound in tissue $(\lambda)$ in $[\mathrm{m}]$ is dependent on the speed of sound in the tissue $(c)$ in $\left[\frac{m}{s}\right]$ and the frequency of sound entering the tissue $(f)$ in $[\mathrm{Hz}]$ through the equation: $\lambda=\frac{c}{f}$ 
As a sound wave travels through a medium it losses energy through absorption in the media, where the energy can be converted into heat or light and scattering where the energy is redirected (Figure 1-3 A). The total dissipative effects of absorption and scattering of the ultrasound wave is termed as “attenuation” (Figure 1-3 B).

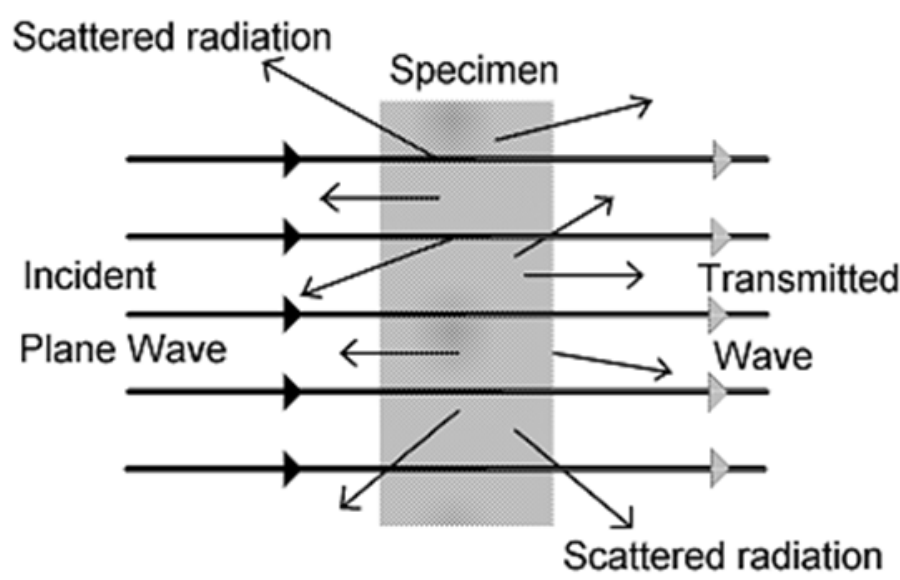

A

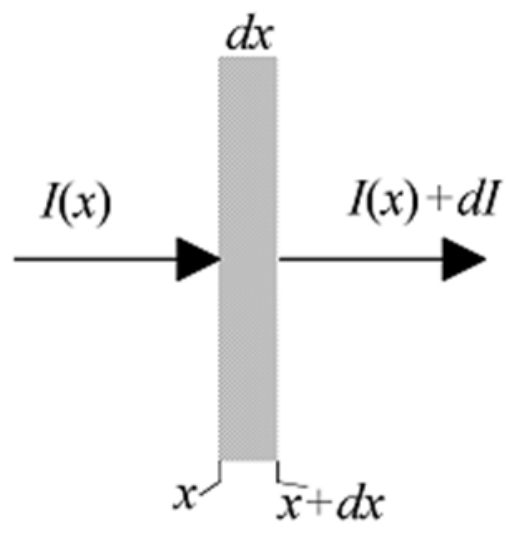

B

Figure 1-3: Absorption and attenuation of ultrasound. (a) Distinguishing between the absorbed, scattered and attenuated waves for a simple plane specimen . (b) Change in intensity for the passage of a plane wave through an incremental distance dx. (Cobbold 2007).

Attenuation is a property of the media, and is frequency dependant.

$\alpha=\alpha_{0} \times f^{n}$

where $\alpha$ in $\left[\frac{N p}{c m \times M H z}\right]$ is the amplitude attenuation coefficient, $f$ is the frequency and $\mathrm{n}$ is an exponent between 1-2. The exponent $n$ is based on the material, so attenuation will change with both frequency and material.

Acoustic impedance is the ratio of acoustic pressure and particle velocity. Characteristic or specific acoustic impedance is a frequency independent material characteristic (in a non viscous medium) and is given by: 
where $Z$ is acoustic impedance [Rayl] , $c_{0}$ is the speed of sound of the medium, and $\rho_{0}$ is the density of the material.

Acoustic power is the rate of energy transfer per unit time in the ultrasound field (Zagzebski 1996). Acoustic intensity is defined as the rate of power transfer per unit area. For a continuous wave the instantaneous intensity is given as:

$I(t)=\frac{p^{2}(\mathrm{t})}{\rho c}$

and the time average intensity is:

$I_{T A}=\frac{p_{0}^{2}}{2 \rho c}$

where $p_{0}$ is the pressure amplitude at a given location in space, $\rho$ is density and $c$ is the speed of sound in the medium. If the wave is pulsed, the time averaged intensity is weighted over the entire cycle, Therefore for a pulsed wave the time averaged intensity is derived from the pulse averaged intensity $I_{P A}$ (Preston 1991)

$I_{P A}=\frac{p_{0}^{2}}{2 \rho c}$

$I_{T A}=\frac{p_{0}^{2}}{2 \rho c} \times D C$

where $D C$ is the duty cycle that is defined as the pulse width divided by the pulse repetition period.

For biomedical applications of ultrasound more descriptive intensities may be required. The United States FDA (Food and Drug Administration) may require spatial average temporal average intensity $\left(\mathrm{I}_{\mathrm{SATA}}\right)$. Generally, $\mathrm{I}_{\mathrm{SATA}}$ is the average intensity distributed over the face of the 
transducer or at a given depth of interest. The FDA defines ISATA as (Acevedo and Das-Gupta 2002)

$I_{S A T A}=\frac{1}{A_{6}} \int_{S_{6}} \int I_{T A}(r) d S$

and power is defined as:

$P=\int_{S_{26}} I_{T A}(r) d s$

Both the intensity and the power are integrated over the surface of the active transducer surface area. $\mathrm{S}_{6}$ is the active area for intensity which is defined as the area where the intensity is greater than $-6 \mathrm{~dB}$ or $25 \%$ of the maximum intensity. The active area for power is defined as $\mathrm{S}_{26}$ where the intensity is greater $0.25 \%$ of its maximum $(-26 \mathrm{~dB})$. Using symmetry for a circular transducer, equations 1.9 and 1.10 can be simplified to (Acevedo and Das-Gupta 2002) :

$I_{S A T A}=\frac{2 I_{S P T A}}{r_{6}^{2} * V_{P}^{2}(0)} \int^{r_{6}} V_{P}^{2}(r) r d r$

$P=\frac{2 \pi I_{S P T A}}{V_{P}^{2}(0)} \int^{r_{26}} V_{P}^{2}(r) r d r$

$r_{6}$ and $r_{26}$ refer to the radius at -6 and $-26 \mathrm{~dB}$. $V_{P}(0)$ refers to the maximum hydrophone voltage at a distance $r$ away from the transducer along the acoustic axis while $V_{P}(r)$ refers to the peak voltage at a distance $R \sin \theta$ away from the axis (Figure 1-4). As can be seen from the above equations both intensity and power can be found using either a using a radiation pressure balance to find average power over the transducer facer or a hydrophone to find voltage change over the transducer face. 


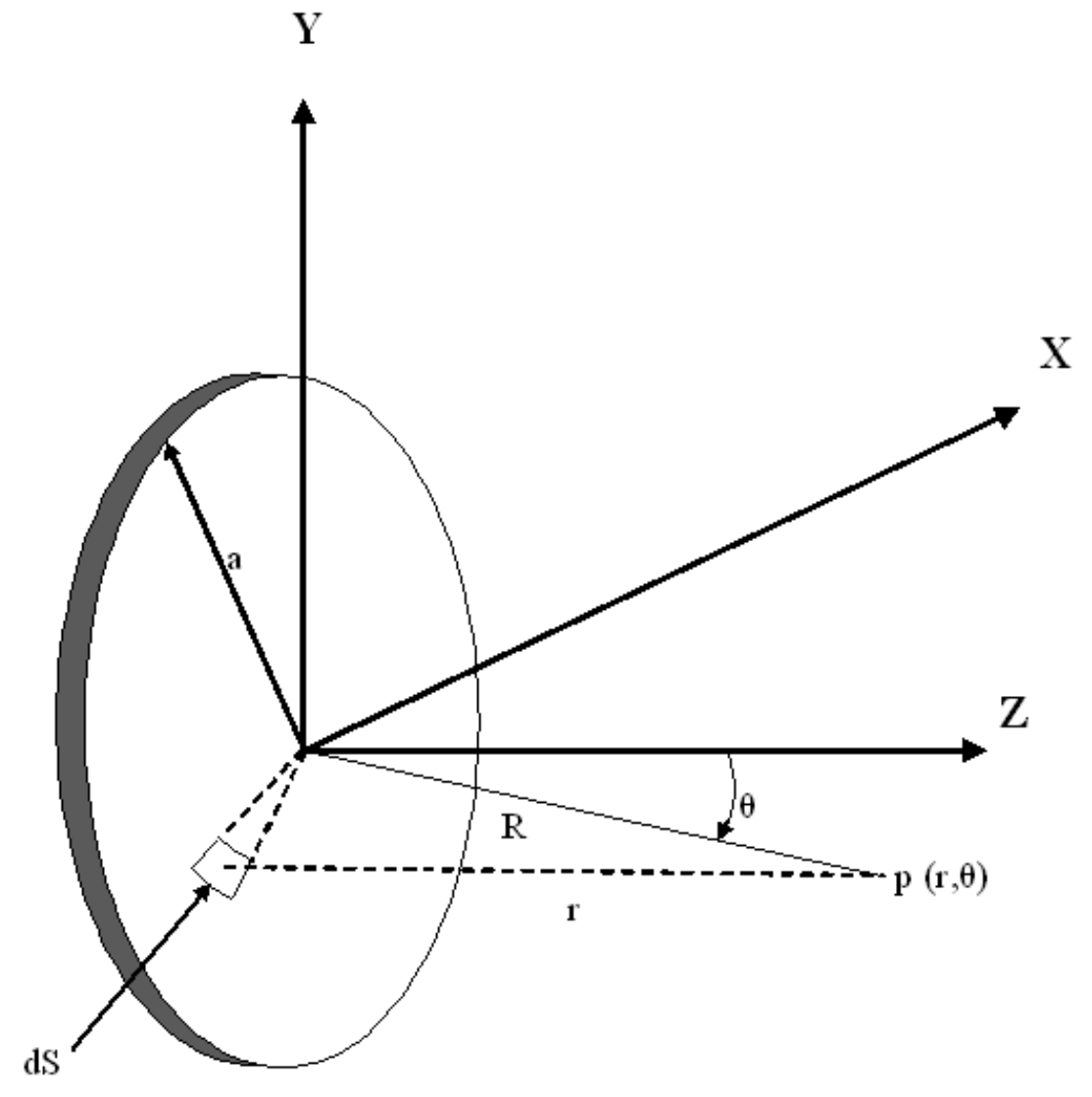

Figure 1-4: Piston Transducer (Zemanek 1971).

Preston further simplifies the method for measuring I IATA (Preston 1991). It can be measured as a function of time averaged intensity integrated over the effective radiating beam area divided by the effective radiating beam area. Then I $_{\mathrm{SATA}}$ is defined as follows (Preston 1991):

$I_{S A T A}=\frac{\int_{\text {Beam area } I_{T A} d A}}{\int_{\text {Beam area }} d A}$

Where $d A$ is the area over the transducer and $\mathrm{I}_{\mathrm{TA}}$ is the intensity over that area in the $X Y$ plane. In practice this means that a large number of equally spaced radial measurements must be taken (greater than 100 points). This leads to the following practical formulation to calculate the ISATA: 
$I_{S A T A}=\frac{\sum I_{T A}}{\text { total number of points }}$

In this thesis, a method based on the equation (1.14) will be used to obtain the $\mathrm{I}_{\mathrm{SATA}}$ from hydrophone measurements.

In most biological applications of ultrasound and ultrasound measurement, the medium is not considered a solid and therefore only the longitudinal component of the wave is considered. However, in biological solids like bone, the shear wave also has a significant effect. In a solid media or at the junction between a fluid and solid media, the intensity of the wave becomes complicated due to mode conversion. Figure 1-5 illustrates that as the ultrasound wave reaches the bone surface (cortical bone), a longitudinal portion of the wave reflects back into the media $\left(R_{l}\right)$, a longitudinal portion of the wave is transmitted through the bone $\left(T_{l}\right)$, and a portion of the wave is converted to a shear wave $\left(T_{s}\right)$. As the wave passes through to the spongy bone this process becomes more complicated because of the part fluid, part solid structure of the bone.

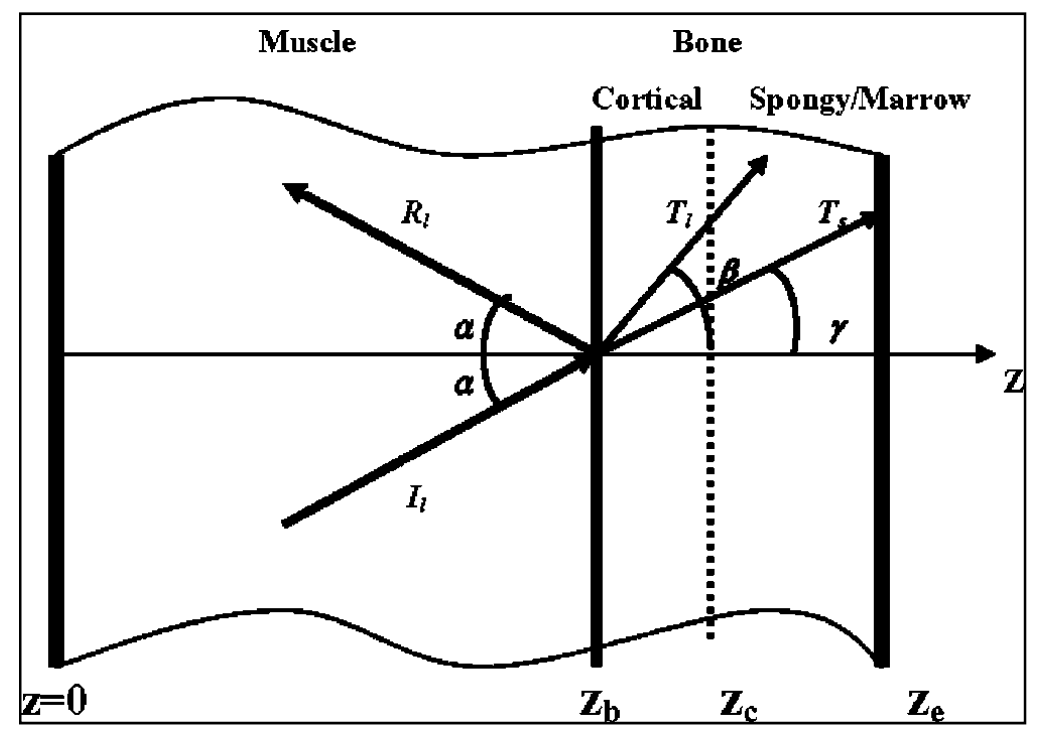

Figure 1-5: Schematic geometry of an ultrasound beam at the muscle/bone interface. Ultrasound propagates through the muscle and then impinges against the muscle/bone interface at a distance of $Z_{b}$. The cortical layer is from $Z_{b}$ to $Z_{c}$ and the spongy/marrow region from $Z_{c}$ to $Z_{e}$. $\alpha, \beta$, and $\gamma$ are the angles of the incident longitudinal wave $\left(I_{1}\right)$, the refracted longitudinal wave $\left(T_{1}\right)$, and the refracted shear wave $\left(T_{s}\right)$, respectively (Liauh et al 2004). 
For bone, the longitudinal speed of sound is approximately 2.5 larger than the shear speed of sound. In the context of ultrasound, bone is a complex material. Some sections of bone are primarily a solid material with small spongy particles within the composite, while other sections of bone are primarily soft tissue with hard particle within it. This means that even at low intensities the interaction between the ultrasound and bone can be complicated.

\subsection{Ultrasound and Bone}

\subsubsection{The History of Ultrasound in Bone Healing}

Early ultrasound was used to detect submarines in 1926 (Schortinghuis et al 2003). As early as 1927 ultrasound was discovered to have biological effects (ter Haar 2007). Fish, put in the path of ultrasound, were found dead (Schortinghuis et al 2003). In addition, investigators experienced extreme pain when they put their hands in the path of the high-power ultrasound.

The earliest biomedical uses of ultrasound suggested its therapeutic attributes. Therapeutic applications of ultrasound predated its diagnostic applications. The original use of ultrasound was as a therapeutic tool to heat tissues (ter Haar 2007). Therapeutically, the addition of heat would increase blood flow, helping to heal the tissue. It was thought that the mechanical wave of ultrasound would stimulate tissue (Schortinghuis et al 2003).

The first use of therapeutic ultrasound on humans was suggested by Pohlman in 1938 (Schortinghuis et al 2003). He established a treatment regime using a frequency of $800 \mathrm{kHz}$ and an intensity of $4-5 \mathrm{~W} / \mathrm{cm}^{2}$ for $10 \mathrm{~min} /$ day for about 10 days. This treatment regime was chosen because it was below the pain threshold, above which a patient would experience burning pain. This type of treatment was mainly used for muscular pain; however, it was used to treat a variety of ailments. 
By 1950 several research groups, including Buchtala, Barth, Demmel, DeForest, Maintz and Ardan (Schortinghuis et al 2003, Claes and Willie 2007), found that ultrasound was affecting the bone. Studies by these groups found mixed results. The intensities used in their experiments ranged between $500 \mathrm{~mW} / \mathrm{cm}^{2}-25000 \mathrm{~mW} / \mathrm{cm}^{2}$. Some groups found ultrasound to cause bone defects, while other groups found that ultrasound given below the pain threshold could stimulate bone growth. During the earliest years of research consistent methods for recording therapeutic doses were not established, and therefore comparison of the results difficult. In addition, intensities that were high enough to stimulate callus formation would later cause the bone to necrotize and die. To further reduce the dose, researchers began to pulse the ultrasound waves.

In 1983, Duarte (Duarte 1983), used a low intensity pulsed ultrasound wave to reduce the detrimental treatment effects on bone. Ultrasound treatments were applied with frequencies of 1.65 and $4.93 \mathrm{MHz}$ with intensities of 49.6 and $57 \mathrm{~mW} / \mathrm{cm}^{2}$ respectively. The transducers were pulsed at $5 \mu \mathrm{s}$, and a repetition frequency of $1 \mathrm{kHz}$. The treatment was applied for $15 \mathrm{~min}$ each day. The treated fractured rabbit fibula and femur showed the area of bone growth increased more rapidly with ultrasound treatments for the first 10-12 days (compared to an untreated control), thus increasing the speed of bone growth (Figure 1-6). In addition, Duarte also noted that the change in frequency did not appear to significantly effect on the outcome; noting that the increased frequency would only effect the intensity through attenuation. Even with changes in frequency, Duarte found significant increase in the rate of bone formation using the ultrasound treatment compared to an untreated control. 

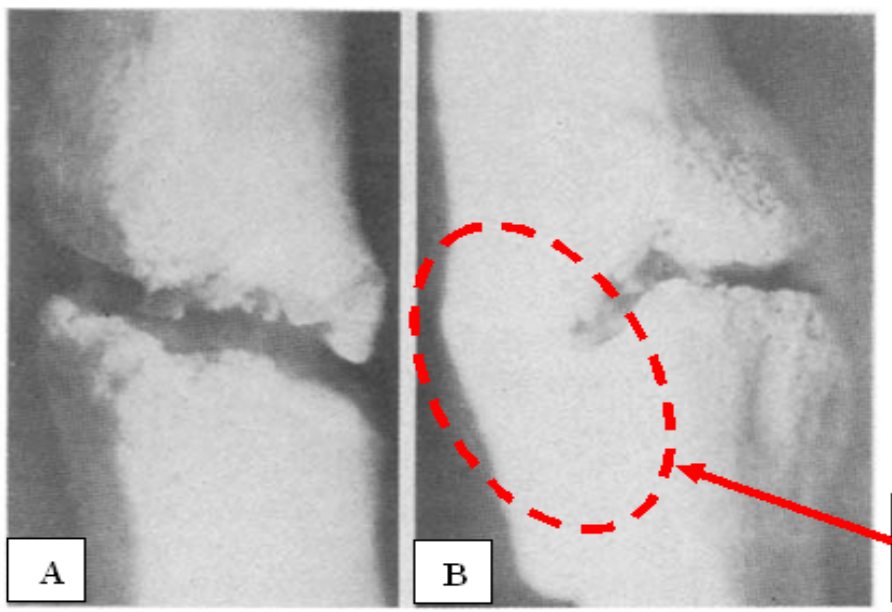

Improved callus formation

Figure 1-6: A) Control limb - no union has formed B) Ultrasound treated limb - significant callus formation (Duarte 1983).

\subsubsection{Low Intensity Pulsed Ultrasound (LIPUS)}

From 1983 to present there have been multiple in vivo, in vitro and clinical Low Intensity Pulsed UltraSound (LIPUS) studies. There have been more than 100 peer reviewed articles (SCOPUS search - ultrasound bone low intensity) studying the effect of LIPUS on bone fractures in animals, estimating healing times, bone strength, number of treatments and thermal effects (Claes and Willie 2007). There have been several phase-I clinical studies on the effects of LIPUS on bone healing, with up to a $40 \%$ improvement in bone healing time for fresh fractures (tibia, radius and scaphoid) and an 85\% improvement in bone healing time in the case of non-unions (based on no additional treatment) (Claes and Willie 2007, Bandow et al 2007, Gebauer et al 2005, Pounder and Harrison 2008, Warden et al 2006, Ricardo 2006, Qin et al 2006, Heckman et al 1994, Kristiansen et al 1997). According to Warden et al (Warden et al 2000), LIPUS is now widely available to promote both fresh fracture and non-union bone healing. Further, Warden suggests not only does LIPUS improve healing rates but also reduces the overall medical cost. 
In 1994 the first therapeutic LIPUS device was approved by the FDA for clinical use with fresh fractures (Exogen ${ }^{\circledR}$ Bone Healing System, Smith \& Nephew Inc., Memphis, TN) (Erdogan and Esen 2009, Siska et al 2008). Further, in 2000, the range of applications increased to include non-unions (Siska et al 2008). Officially LIPUS settings are set to 20 min treatment per day of a 1.5 MHz sine wave repeated at $1 \mathrm{kHz}$ at $\mathrm{I}_{\mathrm{SATA}}=30 \mathrm{~mW} / \mathrm{cm}^{2}$ with a pulse width of $200 \mu \mathrm{s}$ (Claes and Willie 2007, Pounder and Harrison 2008). Due to the prevalence of the Exogen ${ }^{\circledR}$ device, these LIPUS settings are often used as a standard.

Several different LIPUS treatment regimes were tested for effectiveness. Early experiments by Dyson and Brooks (Dyson and Brookes 1983) treated fractures at spatial average temporal peak intensity ( $\mathrm{I}_{\mathrm{SATP}}$ ) of $500 \mathrm{~mW} / \mathrm{cm}^{2}$ with either 1.5 or $3 \mathrm{MHz}$, with a duty cycle of 20\%, 5 min each day, for 4 consecutive days, and varied over the treatment week (ie. treatment group 1 treated for 4 consecutive weeks, treatment group 2 treated week one only, treatment group 3 treated only during week 2, etc.). They found that the therapeutic effect peaked during the first two weeks treatment, during the earlier stages of healing (before the development of hard callus). Additional animal studies further established dose rates. Wang et al. (Wang et al 1994) treated rats with LIPUS at two different frequencies of 0.5 and $1.5 \mathrm{MHz}$ with $15 \mathrm{~min} /$ day treatment for 10 of 15 days after fracture. The study results showed significant improvement in torsional strength and stiffness of bone only for the $1.5 \mathrm{MHz}$ treated fractures. Yang et al. (Yang et al 1996) tested fractures treated with $\mathrm{I}_{\mathrm{SATA}}=50 \mathrm{~mW} / \mathrm{cm}^{2}$ or $100 \mathrm{~mW} / \mathrm{cm}^{2}$ on rabbit tibia. Only the fractured limb treated with $50 \mathrm{~mW} / \mathrm{cm}^{2}$ healed stronger than the control with higher torsional strength and stiffness. Tsai et al. (Tsai et al 1992) applied both $500 \mathrm{~mW} / \mathrm{cm}^{2}$ and $1000 \mathrm{~mW} / \mathrm{cm}^{2}$ for 5,15 , and 25 minutes/day and found that only fractures treated with 500 $\mathrm{mW} / \mathrm{cm}^{2}$ had greater mineral deposition than those treated with $1000 \mathrm{~mW} / \mathrm{cm}^{2}$ (type of intensity 
not mentioned). Fractures treated with $100 \mathrm{~mW} / \mathrm{cm}^{2}$ showed suppressed bone formation. Diagnostic intensity levels of ultrasound were tested when Heyleli et al. (Heybeli et al 2002) applied treatment of $11.8 \mathrm{~mW} / \mathrm{cm}^{2}$ (type of intensity not specified) and $7.5 \mathrm{MHz}$ only once every 5th day for up to 4 weeks. Even these low intensities of ultrasound showed improved bone healing. Heybeli notes that improvements in fracture healing were most apparent in the first two weeks agreeing with earlier results. From 1983 to present, the most effective fracture treatment used lower intensities while higher intensities were found to be less effective.

The healing stages over which treatment is given can also change the ultimate healing strength of the bone. Similar to Dyson and Brooks, Azuma et al. (Azuma et al 2001) applied a standard LIPUS dose $\left(30 \mathrm{~mW} / \mathrm{cm}^{2}, 200 \mu\right.$ s pulse at $1 \mathrm{KHz}$ with a frequency of $1.0 \mathrm{MHz}$ for 20 min each day) to rats over a course of 24 days in order to assess the time frame over which LIPUS was most effective. There were four treatment groups. The comprehensive group received treatment over all 24 days. Each of the 3 other groups received 8 consecutive days of treatment in either the first, second or third week of treatment. All groups showed statistically significant improved torsional strength and stiffness over the controls. However, the group which had been treated for the full 24 days showed significantly higher strength and stiffness compared to the 8 day groups, suggesting that LIPUS treatment given over time may have an additive effect.

The LIPUS regime was established due to the market prevalence of the Exogen ${ }^{\circledR}$ device. The LIPUS treatment standards has shown to be effective, however other treatment parameters are being investigated. This may lead to a better understanding of treatment outcomes and their correlations with the treatment parameters. This may lead to improvement of the LIPUS treatment effectiveness. 


\subsubsection{LIPUS Mechanisms of Action in Bone Fracture Healing}

The mechanisms of how ultrasound induces improved bone healing are not well understood. There are multiple theories for biophysical and biological triggers. Leung et al. (Leung et al 2004a) suggests that the biophysical mechanism of wave mode conversion improves fracture healing. LIPUS generates a longitudinal pressure wave, a portion of which is converted to a transverse or shear wave when it encounters at the soft tissue - bone interface (Leung et al 2004a). The shear wave portion of energy has a significant effect on fracture healing since experimental results showed that the tibia bone healed faster both on the side close to the transducer (the anteromedial surface) and the side farther from the transducer (posterolateral surface) (Leung et al 2004a, Leung et al 2004b). An alternate biophysical mechanism of action is nanoscale motion as suggested by Claes et al. (Claes and Willie 2007) supported by Greenleaf et al. (Greenleaf et al 1996). Local changes in pressure may create a biophysical environment that mimics Wolff's law on a microscopic scale. Pounder and Harrison (Pounder and Harrison 2008), based on Tang's (Tang et al 2004) work, suggest that ultrasound stimulates integrins on the cell surface which then promote bone healing. The combined forces of LIPUS sensitize the cells to shear stress (Pounder and Harrison 2008). Mccormick et al (Mccormick et al 2006) showed that exposure LIPUS alone do not alter cell morphology. Based on this work Pounder and Harrison suggest that since the cell itself does not change morphology under LIPUS treatment, it must be mechanoreceptors on the cell surface than induce changes (Pounder and Harrison 2008). Although individually all of these scenarios are possible, most likely ultrasound mechanisms are a combination of many factors both biophysical and biological.

Cell based models are used to better understand the mechanisms of action for LIPUS. Understanding how ultrasound affects specific biological may lead to a greater understanding of 
how to further improve bone healing. Pounder and Harrison (Pounder and Harrison 2008) suggest that the increase in mechanical strength of the fracture callus is due to accelerated mineralization at the callus site. Several cellular expressions seem to be associated with this accelerated mineralization (Pounder and Harrison 2008). Alkaline Phosphatase (ALP) enhances mineralization while Matrix MetalloProteinase-13 (MMP-13) helps to convert soft callus into hard callus (Unsworth et al 2007). Osteocalsin, a non-collagenous protein, regulates the amount of calcium in its environment. Vascular Endothelial Growth Factor (VEGF) regulates angiogenesis and endochondral ossification. Cyclo-oxygenase 2 (COX-2) is recruited to inflammation sites and produces pro-inflammatory prostaglandins which aid in healing (Simon et al 2002). Increases in prostaglandin (PGE2) are directly related to in vitro mineralization (Pounder and Harrison 2008). Nitric Oxide (NO) is an essential signal to convert a mechanical signal to a biological one (Turner et al 1996, Fox et al 1996). These biological markers are all associated with bone healing in-vivo and in cell cultures. When ultrasound treatments are applied to cell cultures, increased expression of these biological markers may be associated with in-vivo improvements in fracture healing.

With clinical LIPUS settings, Leung et al showed that osteocalsin, alkaline phosphatases, and VEGF in the supernatant of cell cultures increase after 2-4 days of $20 \mathrm{~min} /$ day of stimulation (human periostial cells) (Leung et al 2004a). Also, alizarin red staining showed improved calcium nodule formation after 4 weeks of daily ultrasound treatments (Figure 1-7). Tang et al. (Tang et al 2004) found that ultrasound stimulated integrin expressions with in turn enhanced the expression of COX-2. 


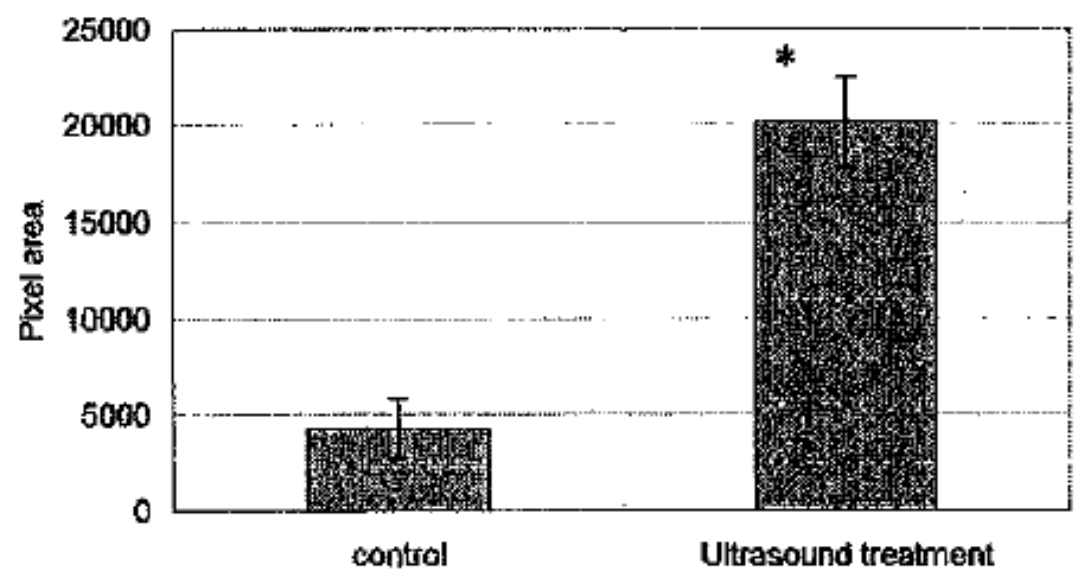

Figure 1-7: The effect of LIPUS treatment on calcium nodule formation of human periosteal cells after 4 weeks of treatment. Image analysis shows the difference in color intensity between the treatment and control group. The asterisk indicates there was a statistically significant difference $(\mathbf{p}<0.001)$. (Error bar $=1$ standard deviation.) (Leung et al 2004a).

With clinical LIPUS settings, Unsworth et al. (Unsworth et al 2007) demonstrated that after 10 days of daily ultrasound stimulation of MC3T3 -E1 mouse osteoblast cells, alkaline phosphatase protein and MMP-13 increased expressions when compared with the control. In addition, compared to the control significant levels of mineralization were seen on days 10, 20 and 25. Figure 1-8 shows that cell cultures show a statistically significant difference in mineralization by day 10 of culture.

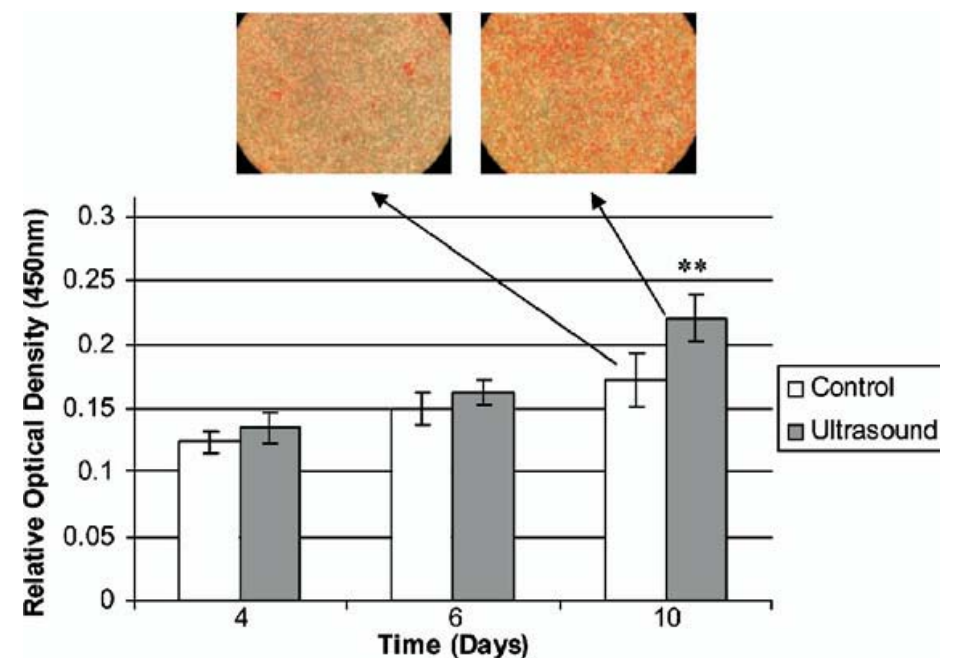

Figure 1-8: Alizarin red staining in cultures of MC3T3-E1. Significant differences were observed at days 10, 20 and 25. Asterisks represent a significant increase ( $\left.\mathbf{p} \_0.005\right)$ vs. control cultures. Results are expressed as mean of six replicates error bar $=$ standard deviation (Unsworth et al 2007). 
Reher et al. (Reher et al 2002) applied a variation of ultrasound signals to human derived osteoblast cells (mandible derived). The $\mathrm{I}_{\mathrm{SATA}}=5,15,30$, and $50 \mathrm{~mW} / \mathrm{cm}^{2}$ at a frequency of 45 $\mathrm{kHz}$ and $20-200 \mathrm{~mW} / \mathrm{cm}^{2}$ on a $1 \mathrm{MHz}$ system with a duty cycles of $20 \%$. Although both frequencies produced increased levels of NO and PGE2, the lower frequency system produced more effective results. Harle et al. (Harle et al 2001) in an attempt to find optimal ultrasound settings for cellular response, applied at 120, 390 and $1490 \mathrm{~mW} / \mathrm{cm}^{2}$ at $3 \mathrm{MHz}$ to MG63 (human derived osteosarcoma cells). Although alkaline phosphatase increased as intensity increased, osteopontin was down regulated, showing that ultrasound may effect different cellular expressions differently (Harle et al 2001). Ultrasound treated cells cultures have shown improved cellular expressions in ALP, MMP-13, COX-2 and PGE2, which improve calcification compared to controls for multiple cell based models through the application of different ultrasound frequencies and intensities.

\subsection{Bones}

\subsubsection{Bone function (Bartel 2006)}

Bone is a composite of organic and inorganic elements. Bone is approximately $30 \%$ collagen which is organic in origin and about $60 \%$ apatite which is inorganic in origin and about $10 \%$ water. Of the organic elements of the bone about $95 \%$ are collagen in origin while the remainder is non-collagen proteins. The non-collagens are primarily proteins like osteocalcin, osteonectin, osteopontin and bone sailoprotein. The inorganic apatite of the bone is comprised of hydroxyapatite containing calcium phosphate, calcium carbonate, calcium fluoride, calcium 
hydroxide and citrate (usually considered an impure hydroxyapatite). The inorganic apatite of bone within the collagen matrix is also called a mineralized matrix.

Figure 1-9 shows minerals within the rod shaped collagen fibrils, making up the mineralized matrix. The collagen fibrils are approximately $300 \mathrm{~nm}$ long and $1.5 \mathrm{~nm}$ in diameter (Figure 1-9 D). Mineralization is thought to start in the holes between the collagen molecules, and then progress to fill up the pores between the molecules. Non collagenous proteins such as alkaline phosphatase, osteopontin and osteocalcin are also found in the spaces between collagen molecules. The mineralized collagen fibril is then either formed into a lamellar layer in which all the collagen fibrils are unidirectional (Figure 1-9) or woven bone where the placement collagen fibrils is random (Figure 1-10). Figure 1-10 is an electron micrograph of a primary cell culture of MC3T3-E1 mouse osteoblast cells showing the collagen fibrils and mineralized matrix vesicles.

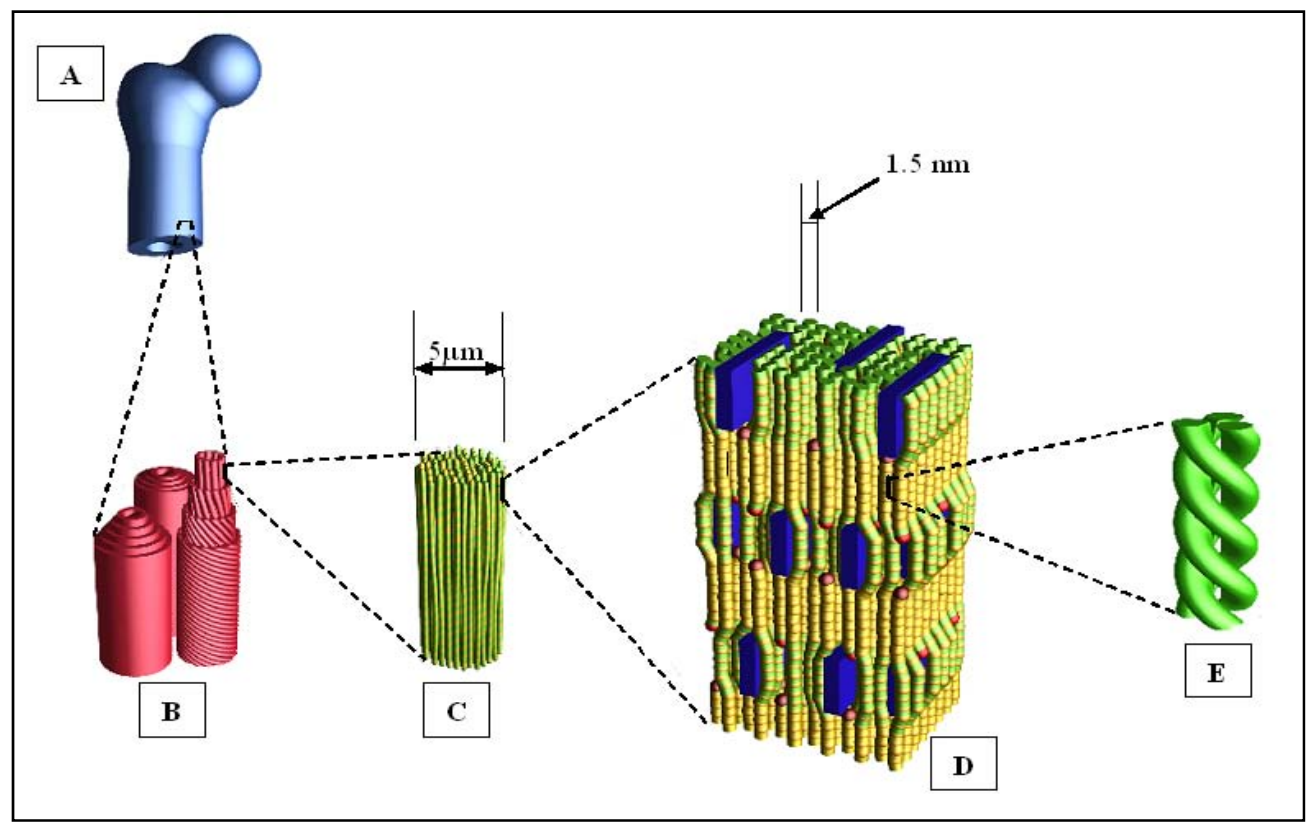

Figure 1-9: Hierarchical structures in bone: (a) Femur (b) Osteons ( $100 \mu \mathrm{m})$ (c) Collagen fiber $(\sim 5 \mu \mathrm{m}$ in diameter) consisting of bundles of collagen fibrils ( $500 \mathrm{~nm}$ in diameter each). (d) Striped collagen fibril consisting of a staggered arrangement of collagen molecules $(\sim 1.5 \mathrm{~nm}$ in diameter, $300 \mathrm{~nm}$ long) with embedded minerals ( 2-20 nm lat.,15 nm wide, $30 \mathrm{~nm}$ long.). (e) Collagen molecule triple helix (Buger 2008). 


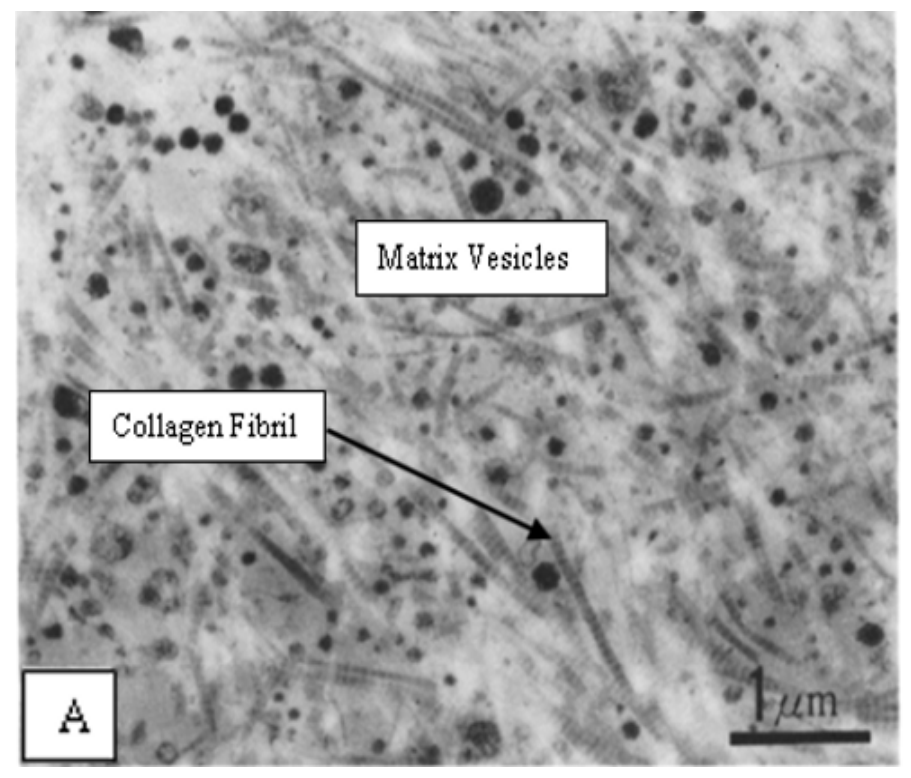

Figure 1-10: Mineral deposits in a primary MC3T3-E1 cultures after 24 days in culture mineralized matrix vesicles among collagen fibrils (Sudo et al 1983).

There are three types of bone formation: endochondral ossification, intramembranous ossification and appositional ossification (Erdogan and Esen 2009, Bostrom et al 2000). Endochondral ossification occurs when cartilage tissue changes into immature bone, then osteoblasts change the immature bone into mature lamellar bone. Intramembranous ossification develops bone without the presence of cartilage when pre-osteoblasts change into osteoblasts and deposit spicules of organic bone matrix (these spicules are deposited onto the periosteum during fracture healing). Appositional ossification begins when osteoblasts align on existing bone surface to form osteoids and layers of lamellae (Erdogan and Esen 2009, Bostrom et al 2000). Most fracture healing involves intramembranous and endochondral ossification (Bostrom et al 2000).

There are five overlapping stages of bone healing after a fracture: hematoma and inflammation stage, angiogenesis and cartilage formation, cartilage calcification, and bone removal and remodeling (Bostrom et al 2000). Immediately after a fracture a hematoma forms 
and provides molecules that signal the initiation of cellular events needed for fracture healing (Figure 1-11 Day 3). Angiogenesis and cartilage formation occur in the first 7-10 days after fracture. During this stage cartilage and new capillaries begin to form next to the periosteum (Figure 1-11 Day 7). About 10 days after fracture, the cartilage should surround the fracture and begin to calcify. At this point, there will be both intramembranous ossification (where preosteoblasts change into bone cells and calcify - flat bones) and endochondral ossification (where cartilage calcifies and changes into bone - fracture healing). The callus will mineralize and lay down organic bone matrix will form in the soft tissue (Figure 1-11 Day 14). Through intramembranous and endochondral ossification the cartilage and soft tissue will become woven bone (Figure 1-11 Day 21). Bone remodeling occurs as woven bone remodels into lamellar bone through osteoclast bone re-absorption and organized osteoblast bone deposition (Bostrom et al 2000).

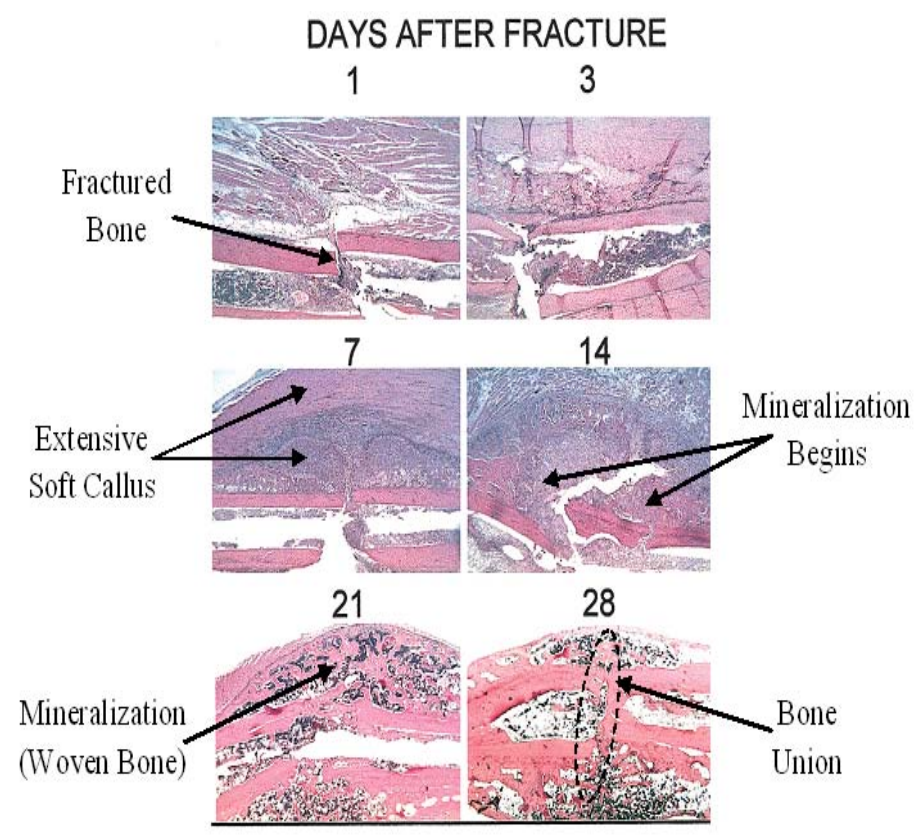

Figure 1-11: Histological analysis of fracture healing. The progression of repair on days 1,3,7,14,21, and 28. On day 7, extensive soft callus is seen forming around the injured bone. At day 14, the soft callus becomes mineralized to form new bone and achieve union by day 21 and 28 (x40) (Stroncek and Reichert 2008). 


\subsubsection{MC3T3-E1 bone cells}

An immortalized mouse calvarial cell line represents only a portion of the bone growth process; the phase of growth associated with calcification. The complete process of bone growth and repair needs a variety of cells. A key component to the reparative phase of healing is mineralization. LIPUS has been shown to be most useful during the reparative phase of bone healing. For this reason, MC3T3-E1 cells have been chosen for this experiment. In MC3T3-E1 cells intramembranous ossification is the primary type of bone formation of interest. This cell line only represents pre-osteoblasts changing into mineralized osteoblasts; therefore endochondral ossification will not be present in this cell line. This model of bone cell mineralization only represents a particular window of bone formation.

There are several distinct physiological stages to MC3T3-E1 bone cell differentiation. The cells first proliferate, then a collagen matrix begins to form (Figure 1-12), and finally calcified mineral matrix begins to form. For bone cell differentiation to occur, either in cell cultures or in the human body, Ascorbic Acid (AA) must be present (Franceschi et al 1994). To further stimulate a calcified mineral matrix to form a source organic phosphate (like $\beta$-glycerol phosphate) must be present. The organic phosphate will allow hydroxyapatite (containing calcium and other minerals) to form between the within the collagen fibers (Figure 1-9 and Figure 1-12) (Wang et al 1999). 


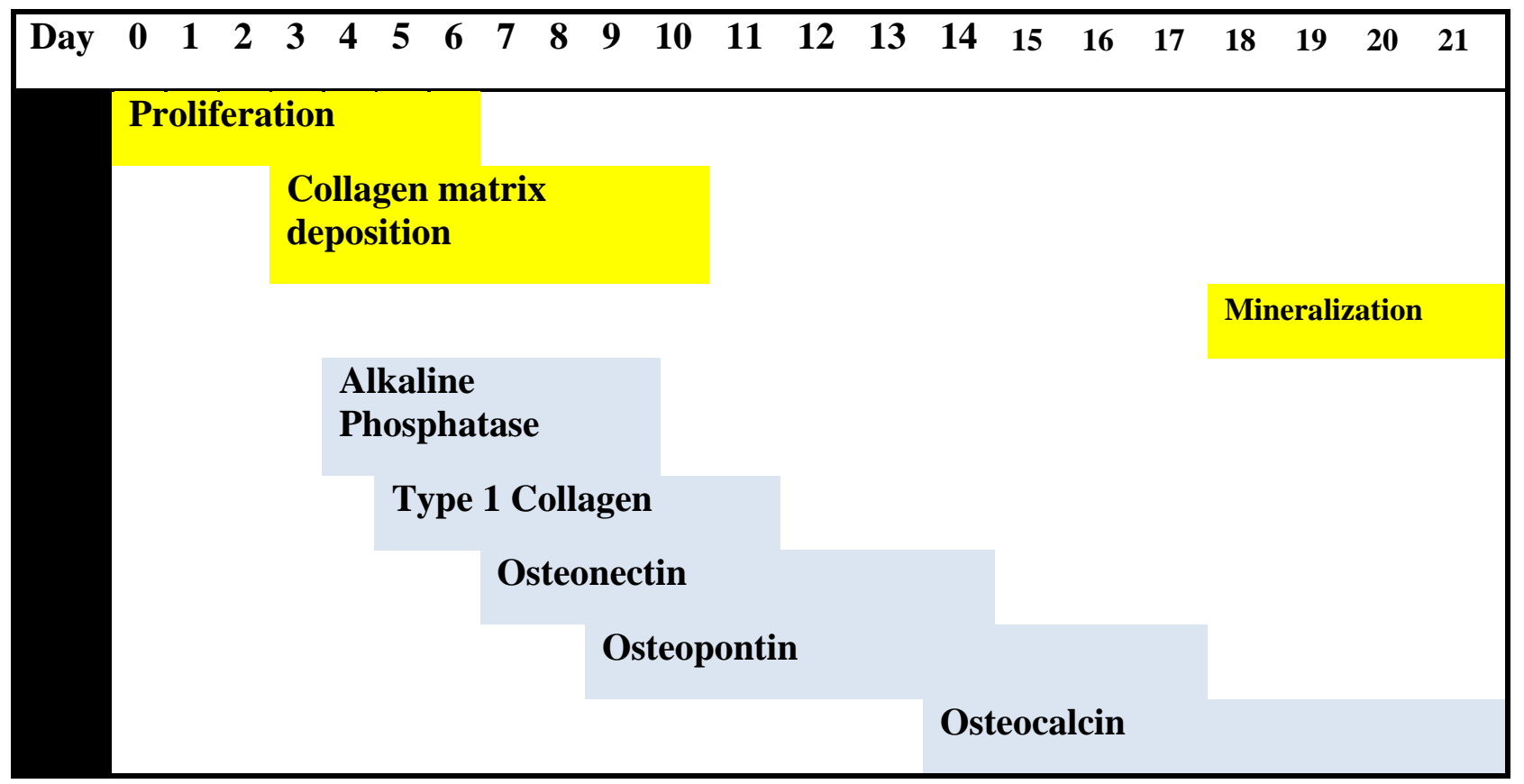

Figure 1-12: Time dependant differentiation of MC3T3 cells. Yellow indicates phenotype changes as the cell differentiates. Blue-Gray indicates protein expressions as the cells differentiates into a mineralized osteoblast (Beck Jr. et al 2000).

\subsubsection{Mineralization}

At a cellular level matrix vesicles provide a location for calcium accumulation.

Mineralization occurs when matrix vesicles (small extracellular packets of minerals) express mineral crystals through their membrane (Anderson 2003). Hydroxyapatite crystals are exposed to extracellular fluid which contains a sufficient amount of $\mathrm{Ca}^{2+}$ (and $\mathrm{PO}_{4}{ }^{3-}$ ) to support continuous crystal proliferation (Anderson 2003). As a result, the apatite rich matrix vesicles are formed preferentially in regions of osteoblast differentiation.

\subsection{Heating}

Leon et al., (Leon et al 1993), while studying the in-vivo temperature distribution in bone, found that after heating bone to $43^{\circ} \mathrm{C}$ for 45 minutes and treated 4 times over 21 days, the bone 
was denser. As can be seen in Figure 1-13, only the heat treated bone shows a significantly thicker callus. Evidence of improved mineralization was also apparent on a microscopic level.

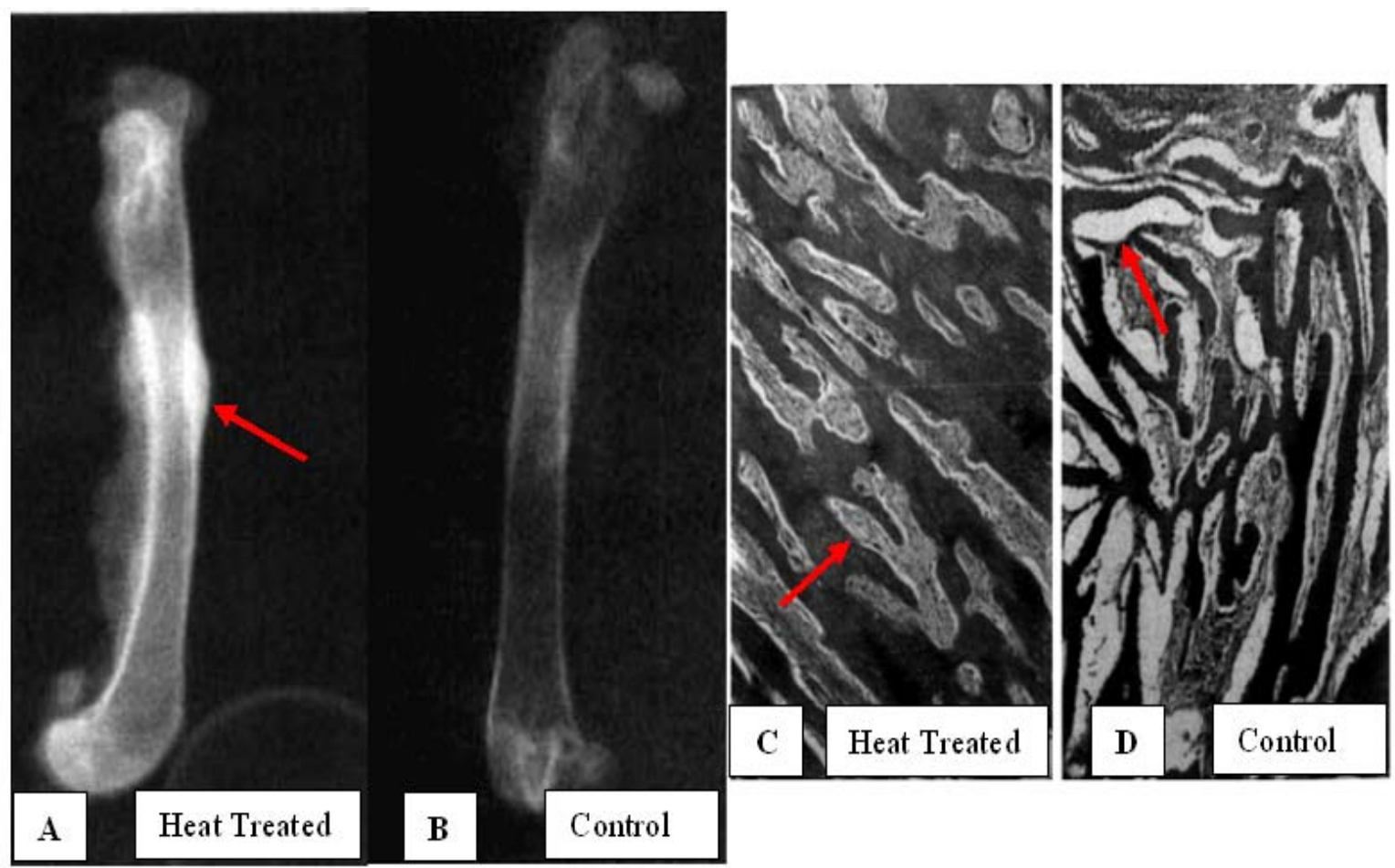

Figure 1-13: A) The arrow indicated that the heat treated bone has significantly thicker bone callus than B) the control bone. C) The arrow indicates that the histological sample show heat treated bone has significantly improved mineral deposits. D) The arrow indicates that the control sample shows an open structure (Leon et al 1993).

According to Flour et al. (Flour et al 1992), a temperature increase to $40^{\circ} \mathrm{C}$ for 24 hours did not significantly change the viability (Figure 1-14) or proliferation (Figure 1-15) of MC3T3, rabbit chondrocytes, rat osteoblasts and osteosarcoma (ROS 17/2.8) cells lines. Flour et al. suggests the critical temperature for viability and proliferation is between $42^{\circ} \mathrm{C}$ and $43^{\circ} \mathrm{C}$ above which cells will not be viable. In a different experiment by Trieb et al. (Trieb et al 2007), SaOS2 osteosarcoma cells showed decreased proliferation when heated to $42^{\circ} \mathrm{C}$ for one hour. This seems to indicate that multiple osteoblast cell groups are tolerant to small temperature changes over a limited time span or a limited thermal dose. 


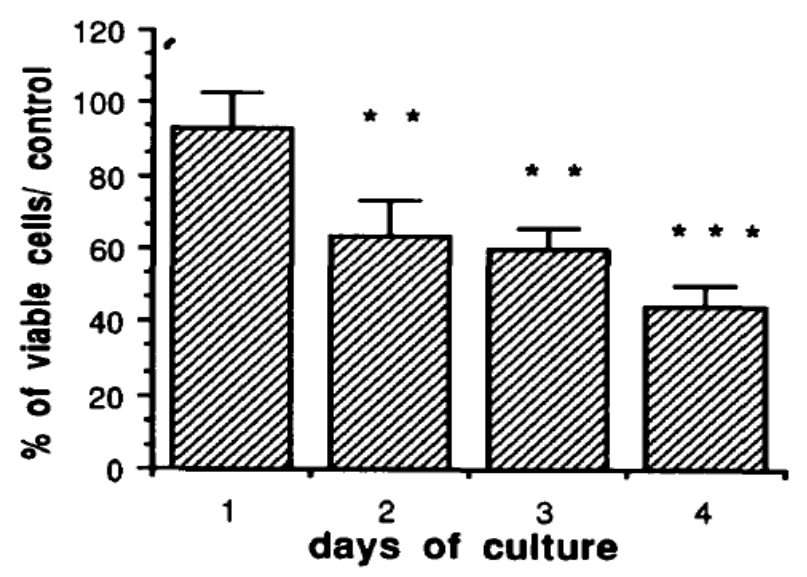

Figure 1-14: Effect of hyperthermia on MC3T3-E1 cell viability. The bars represent the number of days in culture at $40^{\circ} \mathrm{C}$. Cell viability is expressed as a percentage of control cells cultured at $37^{\circ} \mathrm{C}$. ${ }^{*}: \mathbf{P}<0.05$, ${ }^{* *} \mathbf{P}<\mathbf{0 . 0 1},{ }^{* * *}: \mathbf{P}<\mathbf{0 . 0 0 1}$ (Flour et al 1992).

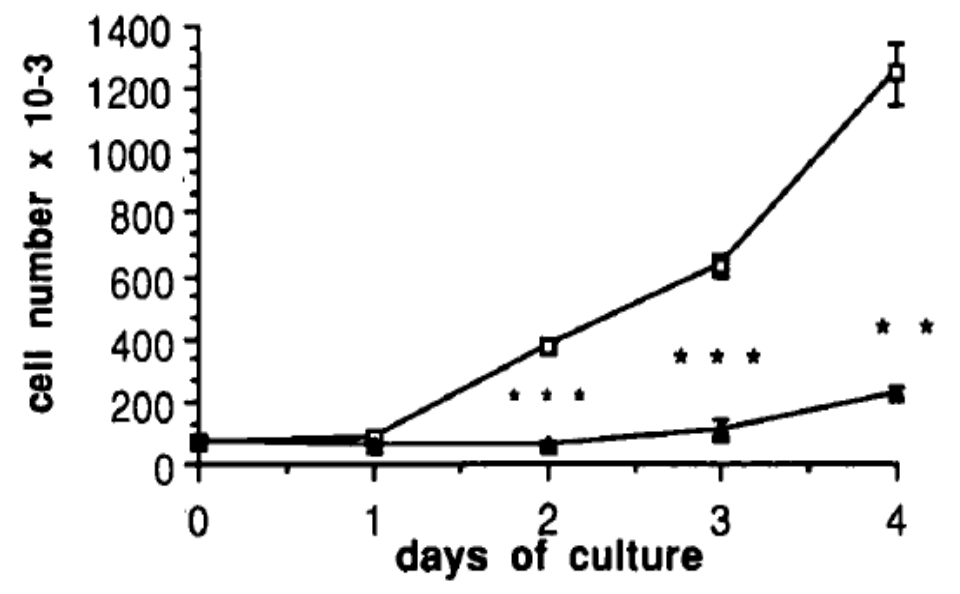

Figure 1-15: Effect of hyperthermia on MC3T3-E1 proliferation. at $37^{\circ} \mathrm{C}(\square)$ and $40^{\circ} \mathrm{C}(\triangle)$. Each value is the mean of three determinations. ${ }^{*}: \mathbf{P}<\mathbf{0 . 0 5},{ }^{* *} \mathbf{P}<0.01,{ }^{* * *}: \mathbf{P}<\mathbf{0 . 0 0 1}$ (Flour et al 1992).

Shui et al. (Shui and Scutt 2001) tested human bone marrow stromal cells (BMSC) in vitro for the effect of heating on mineralization. They found that cells heated for $39-41^{\circ} \mathrm{C}$ for one hour every 3rd day for 21 days (Figure 1-16) and cells heated at $39^{\circ} \mathrm{C}$ for 96 hours that were measured after 10 days of incubation (Figure 1-17), both showed significant increases in calcium mineralization. 


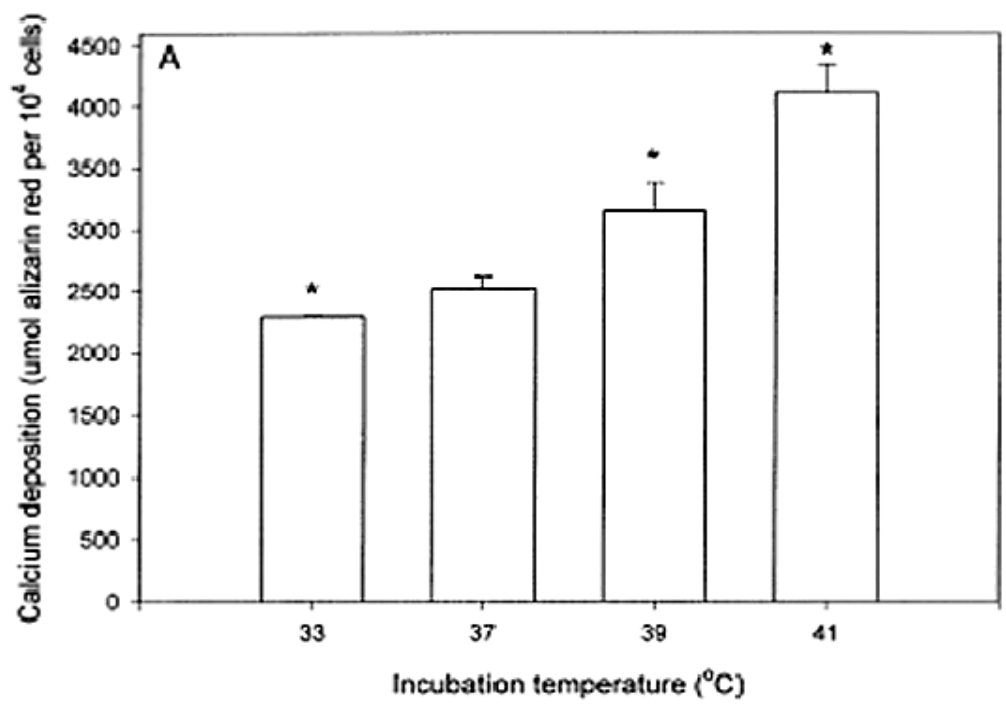

Figure 1-16: Calcium deposition by BMSCs after multiple 1-h exposure to different temperatures. The cells were exposed to different temperatures for $1 \mathrm{~h}$ at intervals of every 3 days. After 21 days the cultures were stopped *:P < 0.05, compared with control $\left(37^{\circ} \mathbf{C}\right)$ (Shui and Scutt 2001$)$.

Although there is not a large volume of research on the effects of low level heating on bone, the research that has been done indicates that increases in temperature of just a few degrees can significantly increase mineralization of both bone and bone cells.

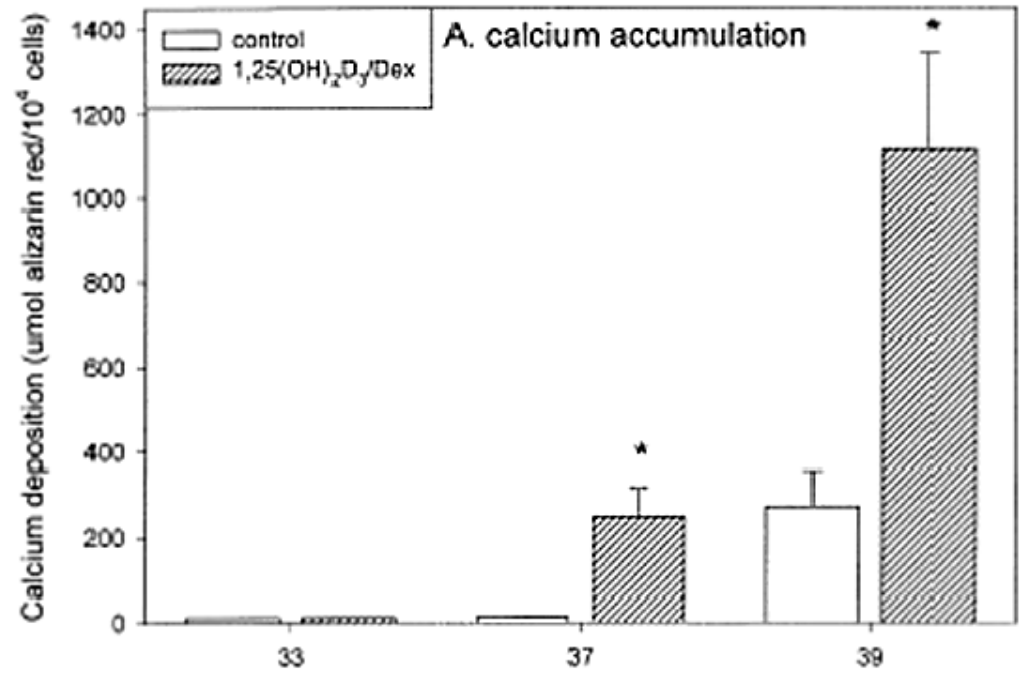

Figure 1-17: Calcium deposition by BMSCs after long-term exposure to low and high temperatures. After incubation at 33,37 , or $39^{\circ} \mathrm{C}$, respectively, for 10 days, the cultures were stopped. $*$ :P $<0.05$, compared with control $\left(37^{\circ} \mathbf{C}\right)$ (Shui and Scutt 2001). 


\subsection{Heating and Ultrasound}

At intensities in the LIPUS range, heat does not seem to be a mechanism of action for enhancing bone mineralization. When applying ultrasound between 50 and $57 \mathrm{~mW} / \mathrm{cm}^{2}$, Duarte (Duarte 1983) notes that the temperature rise, in vivo, was less than $0.01^{\circ} \mathrm{C}$. Chang et al. (Chang et al 2002) tested heating as a possible mechanism for LIPUS. Equal energy doses were applied to rabbit limbs with ostomies. The ultrasound dose $\mathrm{I}=0.5 \mathrm{~W} / \mathrm{cm}^{2}$, microwave heating dose was set at $\mathrm{I}=0.5 \mathrm{~W} / \mathrm{cm}^{2}$. The effect of these energy doses was compared to a control which received no treatment. Only the ultrasound treated limb has statistically significant improvement in bone formation and torsional stiffness. Most recently Leskinen et al. (Leskinen et al 2009) tested the effects of heat and ultrasound on an osteosarcoma cell line. The study looked at temporal average power ranging from 200 to $2000 \mathrm{~mW}\left(\mathrm{I}_{\mathrm{SATA}}=20-200 \mathrm{~mW} / \mathrm{cm}^{2}\right.$, based on a transducer diameter of 25mm) with frequency of $1.035 \mathrm{MHz}$, PRF $1 \mathrm{kHz}$ and DC of 20\%. Cell signaling associated with improved bone formation increased at temperatures above $48^{\circ} \mathrm{C}$ and ultrasound power above $400 \mathrm{~mW}$. The heat and ultrasound treatments were not given concurrently. No examples of low level heat and LIPUS have been found in the literature review.

In most cases of concurrent treatment of heat and ultrasound, the addition of heat is used synergistically to enhance cell death. In the few studies that have examined low level heat, the ultrasound dose is higher than $30 \mathrm{~mW} / \mathrm{cm}^{2}$ and none use bone cells. Kondo (Kondo and Kano 1987) subjects cell cultures to a combination of heat and ultrasound. For this work suspended mouse L fibroblast cells were used. The cells were exposed to $\mathrm{I}_{\mathrm{SPTA}}=3.7 \mathrm{~W} / \mathrm{cm}^{2}\left(\mathrm{I}_{\mathrm{SATA}}=2.7\right.$ $\mathrm{W} / \mathrm{cm}^{2}$ ) for $20 \mathrm{~min}$ with a continuous pulse and a stable temperature of either $37^{\circ} \mathrm{C}$ or $44^{\circ} \mathrm{C}$. Cell death was enhanced with the addition of heating at $44^{\circ} \mathrm{C}$ for $20 \mathrm{~min}$. These results suggest that the combined dose of heat at $44^{\circ} \mathrm{C}$ plus ultrasound with the intensity of $\mathrm{I}_{\mathrm{SPTA}}=3.7 \mathrm{~W} / \mathrm{cm}^{2}$ for 
duration of 20 min will kill cells. Feril et al. (Feril Jr. et al 2002), subjected human leukemic lymphoma (U937) cell cultures to intensities of 0.5 or $1 \mathrm{~W} / \mathrm{cm}^{2}$ at $44^{\circ} \mathrm{C}$ for 10 minutes. While treatment of $44^{\circ} \mathrm{C}$ and $0.5 \mathrm{~W} / \mathrm{cm}^{2}$ showed loss of cell viability, the combined dose of $44^{\circ} \mathrm{C}$ and 1 $\mathrm{W} / \mathrm{cm}^{2}$ showed significant synergistic cell killing. Although high levels of heat plus ultrasound cause cell death, the individual treatments of low level heating and LIPUS seem to improve mineralization in cell cultures.

\subsection{Physics of Light Absorbance: Beer's Law (Workman 1998)}

Many of the changes in bone cell cultures are detected through chemical assays which used changes in light absorbance of the solutions to test for specific changes is concentrations of various substances.

Beer's law is the relationship between sample concentration and the spectrometer response. For the scenario in Figure 1-18, $\mathrm{I}_{0}$ indicated the energy of the incident light source and $I_{1}$ indicates the transmitted energy of the light source. The absorbance is based on the sample concentration.

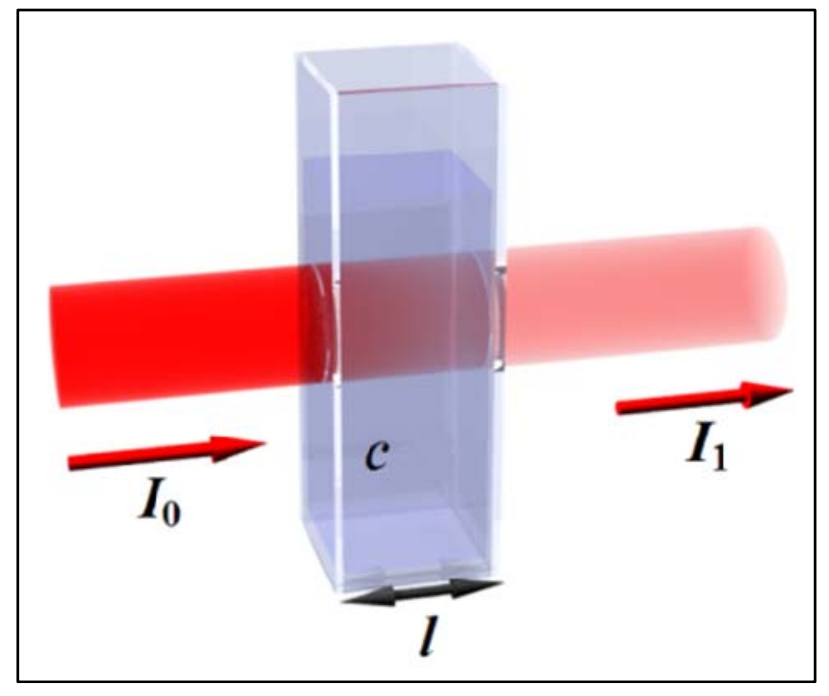

Figure 1-18: Light absorption through a cuvette. 
$A=\varepsilon l c=-\log _{10} \frac{I_{1}}{I_{0}}=-\log _{10}(T)$

Where $\mathrm{A}=$ absorbance, $\mathrm{T}=$ transmission $\varepsilon=$ molar absorptivity $\left(\mathrm{cm}^{-1}\right)$ or $\left(\mathrm{L} \mathrm{mol}^{-1} \mathrm{~cm}^{-1}\right)$ or $\left(\mathrm{mol}^{-1} \mathrm{~cm}^{2}\right)$ and $\mathrm{c}=$ concentration of the sample and $\mathrm{l}=$ =path length. It should be noted that the relationship between absorbance and concentration is linear. In addition, the term optical density has the same definition as absorbance and is frequently used as a synonym.

Beer's law is functionally used to identify different concentrations of substances. This is especially useful when comparing multiple samples to a control. From Figure 1-19, it is clear that Sample A has higher absorbance than Sample B because it has a higher concentration.

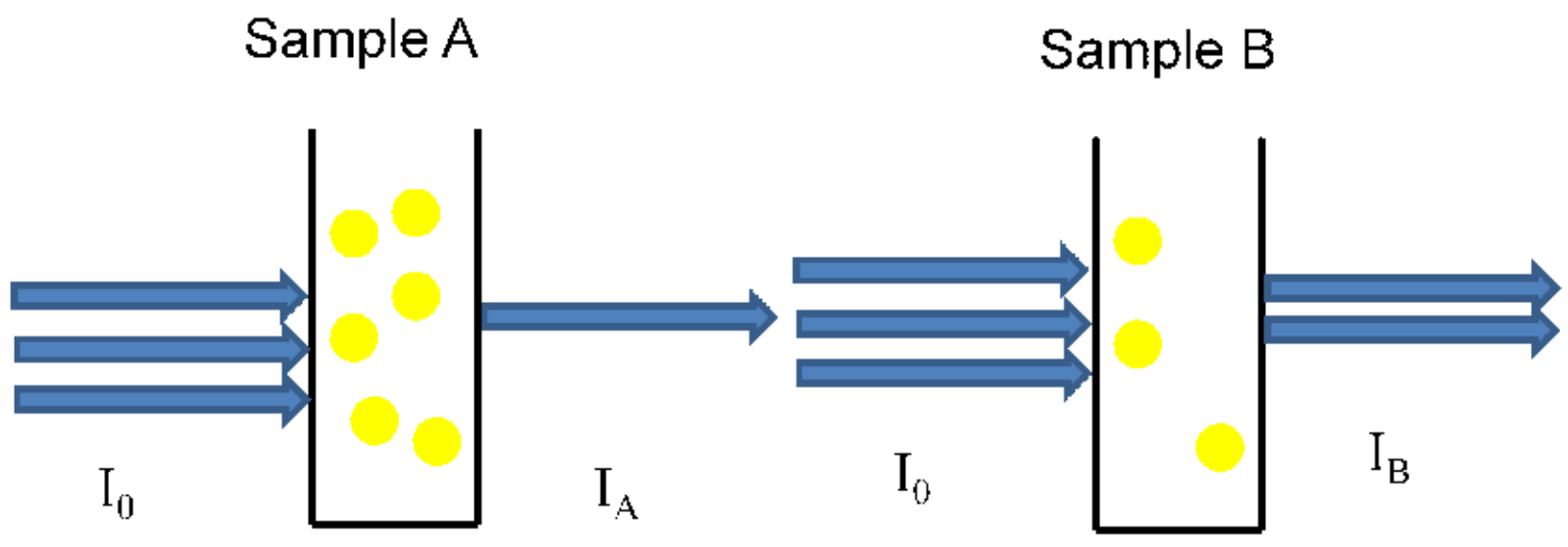

Figure 1-19: Spectrometer measurements using Beer's Law. The absorption of sample $A$ is higher than the absorption of sample $B$ because the concentration of sample $A$ is greater.

\subsection{Alizarin Red (Puchtler et al 1969)}

Alizarin red was originally derived from a madder root and is sometimes called a madder dye. It has been used as a textile dye for over 1500 years. In the $19^{\text {th }}$ century scientists noticed 
that madder dye had an affinity to bone, changing its color to red when in contact with bone. Although this dye is now known to bind to multiple elements in addition to calcium, these elements do not occur with enough concentration to interfere with staining.

Alizarin red is now produced synthetically. Alizarin dye has the chemical formula $\mathrm{C}_{14} \mathrm{H}_{8} \mathrm{O}_{4}$. As seen in Figure 1-20 the 1 and 2-OH dissociation constants allow the calcium to bond with the dye. Alizarin red $-\mathrm{S}$ has the chemical formula $\mathrm{C}_{14} \mathrm{H}_{7} \mathrm{NaO}_{7} \mathrm{~S}$ (Figure 1-21). With this chemical formulation, "the sulfonic acid group ... forms a [bond] with calcium. Once calcium atom reacts with two sulfonic acid groups” (Puchtler et al 1969). The chemical transformation is as follows:

$\left(\mathrm{C}_{14} \mathrm{H}_{8} \mathrm{O}_{4}\right) \mathrm{SO}_{3} \mathrm{Na}+\mathbf{C a C l}_{2} \rightarrow\left(\mathrm{C}_{14} \mathrm{H}_{8} \mathrm{O}_{4} \mathrm{SO}_{3}\right)_{2} \mathbf{C a}+2 \mathrm{NaCl}$

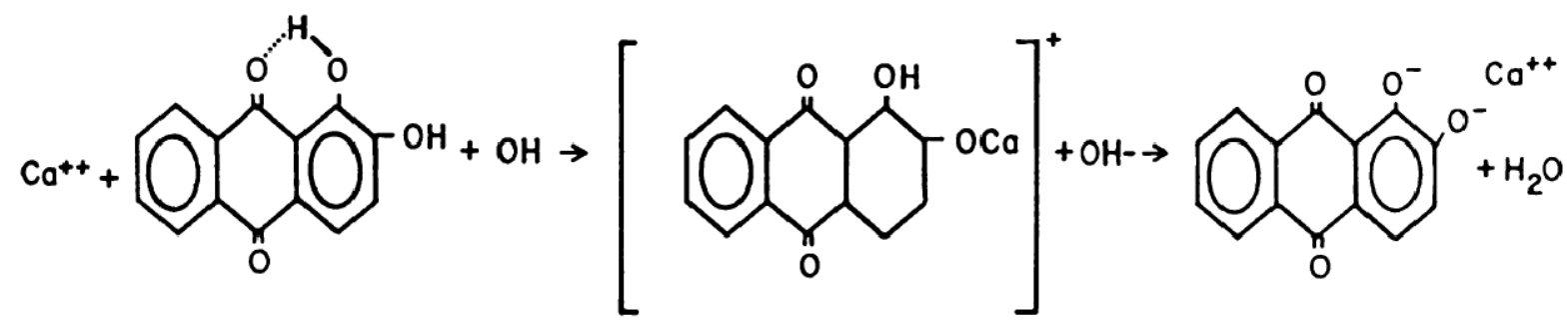

Figure 1-20: Formation of the calcium alizarin red bond (Puchtler et al 1969).

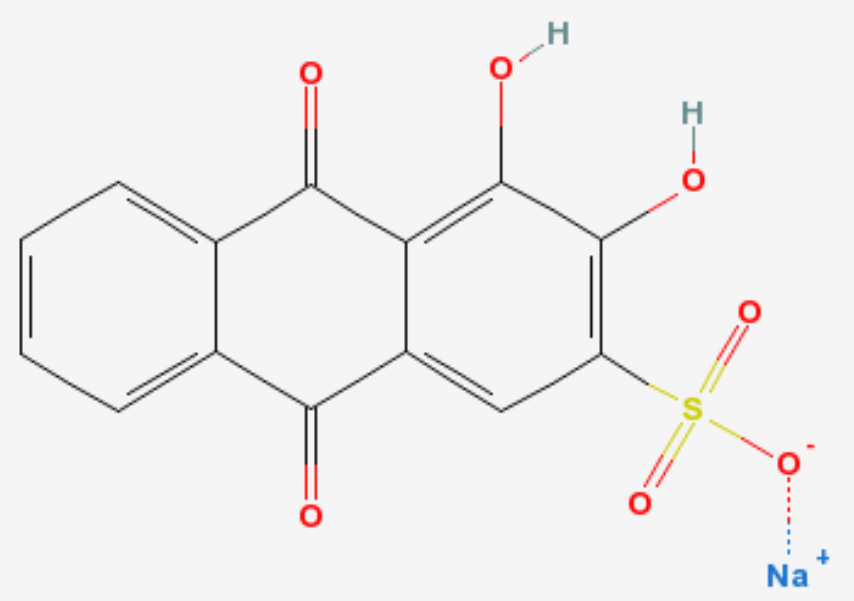

Figure 1-21: Formulation of the alizarin red-S molecule (U.S. National Library of Medicine ). 
Since alizarin forms such regular and consistent bonds with calcium, with 1 mole of alizarin red-S bonding to 2 moles of calcium, it is commonly used in histological and cell culture staining (Scutt et al 2003). From the formula above it is clear that the more calcium that is available the more the stain will be absorbed.

\subsection{Clinical Motivation}

Currently fractures remain a clinical problem across the United States and Canada. In 1995, there were approximately 10 million fractures across the United States (Figure 1-22). In 2003 there were 115456 hospital admissions for musculoskeletal injuries across Canada (Canadian Institute for Health Information (CIHI) ). About 10\% of all hospital admissions are the result of fractured bones. In 2007 in the United States, more than 50\% of all hospital admissions due to injury are fractures (Bergen et al 2008). Fractures remain a significant issue in the Canadian and US health care systems.

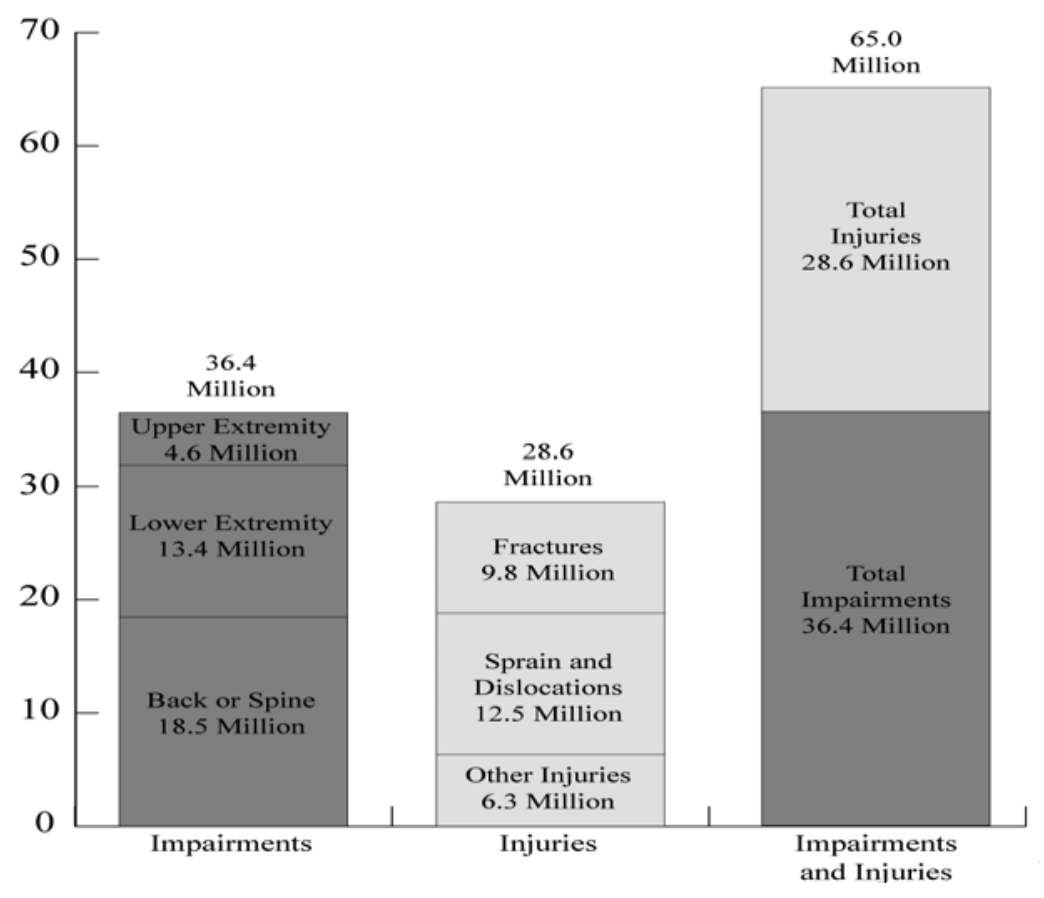

Figure 1-22: Musculoskeletal impairments for 1995 (Bone and Joint Decade 2009). 


\subsection{Hypothesis}

The combined effect of (Low Intensity Pulsed UltraSound) LIPUS and low level heat will have an additive healing effect further promoting bone healing.

\subsubsection{Specific Aims}

The specific aim for this thesis is to show that the addition of ultrasound and low level heat will increase mineralization in bone cell cultures. 


\section{Chapter 2 Exogen ${ }^{\circledR}$ LIPUS Device and its Acoustic}

\section{Characterization}

\subsection{Introduction}

The purpose of this chapter is to confirm the stated acoustic characteristics of the Exogen ${ }^{\circledR}$ LIPUS device (research version) provided by Smith \& Nephew Inc., Memphis, TN. The characteristics tested include; frequency, pulse duration, pulse repetition frequency, duty cycle, effective radiating area, and spatial average temporal average intensity ( ISATA $_{\text {SAT }}$.

This chapter will also address the degree to which a polystyrene cell plate affects the output intensity of the device. Cell cultures are normally grown on polystyrene plates. In most studies s on cell cultures (including this one) the ultrasound is applied through the polystyrene plate to the cell cultures, in which the polystyrene plate may change the energy deposited into the cell cultures.

\subsection{Materials and Methods}

\subsubsection{Exogen ${ }^{\circledR}$ LIPUS Device}

Smith \& Nephew Inc., Memphis, TN. has developed and commercialized a low intensity pulsed ultrasound device for the clinical market. The Exogen 4000+ Bone Healing System ${ }^{\circledR}$ (Figure 2-1 A) is clinically indicated for fresh fractures and non-unions (excluding the scull and spine) in skeletally mature individuals (Smith \& Nephew 2009) (FDA Approvals, 1994, 2000).

The clinical device consists of a single head, flat circular transducer. The transducer settings for the clinical device are $1.5 \pm 5 \% \mathrm{MHz}$ pulse, a pulse width of $200 \pm 10 \% \mu \mathrm{s}$ and a pulse repetition frequency of $1 \pm 10 \% \mathrm{kHz}$. The ultrasound treatment is applied to the skin 
above the fracture site of the stabilized bone. The transducer is coupled to the skin using waterbased ultrasound coupling gel also provided by Smith \& Nephew Inc.
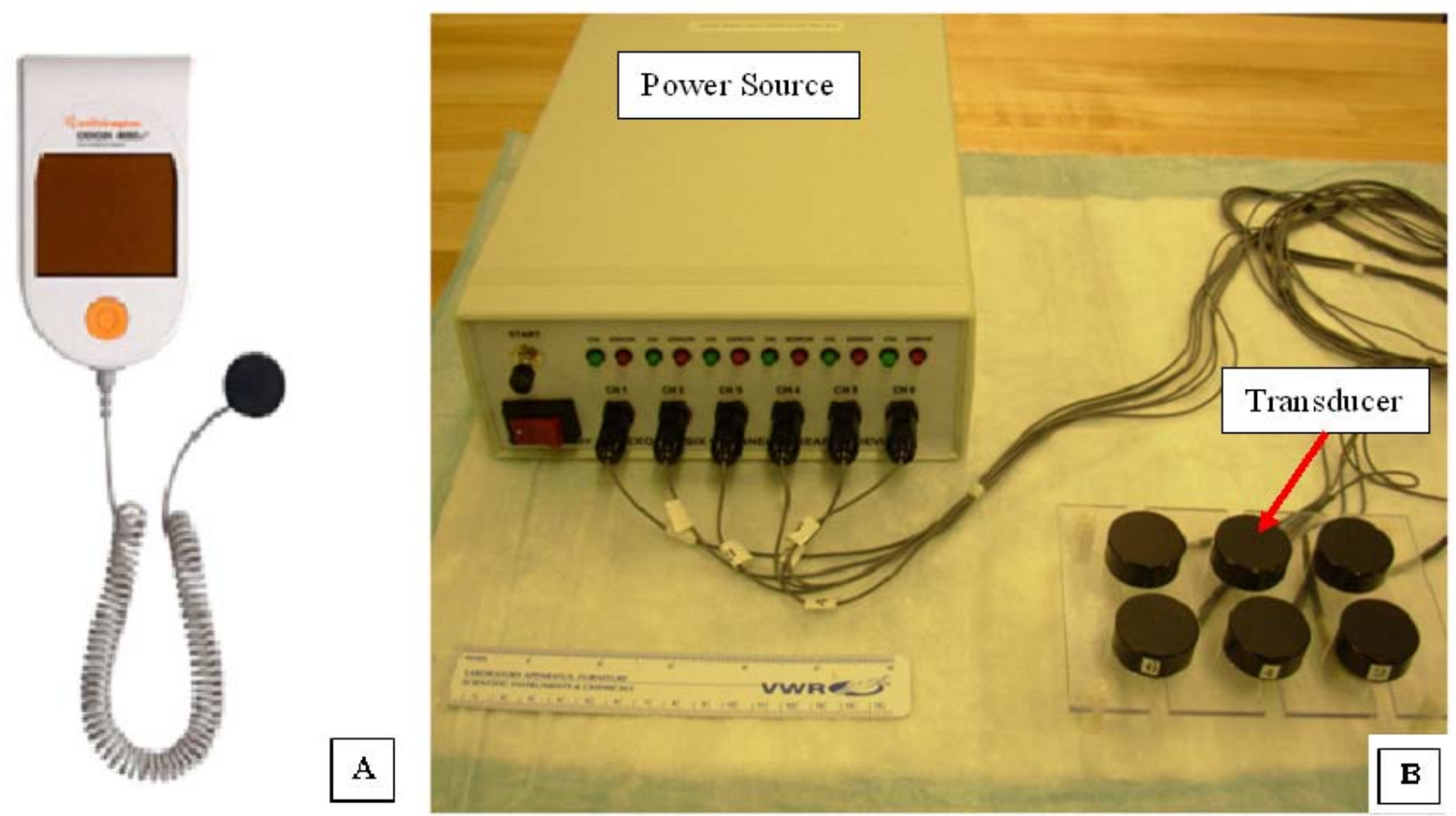

Figure 2-1: A) Exogen ${ }^{\circledR} 4000$ + bone healing clinical system ${ }^{\circledR}$ and B) Exogen ${ }^{\circledR}$ multi-transducer research device with an array of 6 transducers.

Smith \& Nephew Inc., has built a research device using an array of identical transducers with a modified holder (Figure 2-1 B). The Exogen ${ }^{\circledR}$ research device includes 6 x $1.5 \mathrm{MHz}$ transducers, each individually driven by a power source. The array of 6 identical transducers has been designed specifically for use with cell cultures using 6 cell culture plates.

In this chapter, the acoustic output characterization of the Exogen ${ }^{\circledR}$ research device and the effect of polystyrene cell plate on the acoustic output will be presented. 


\subsubsection{Acoustic Output Measurement System}

Acoustic output measurements were made in a water tank using filtered degassed water (Figure 2-2). Hydrophone measurements were made using a Precision Acoustics Calibrated Hydrophone (Precision Acoustics Ltd., Dorchester, Dorset, UK). The hydrophone was moved through the acoustic field using the Daedal Motion System (Parker Hannifin Corp., Electromechanical Automation Division of Daedal, Irwin, PA). The hydrophone voltage measurements were read off a Tektronix TDS 5052 Digital Phosphor Oscilloscope (Tektronix Inc., Beaverton, OR, USA). In all measurements, a single $1.5 \mathrm{MHz}$ transducer on the Exogen ${ }^{\circledR}$ research device was used to provide the ultrasound signal (Figure 2-1, indicated with a red arrow). The comparison of the single transducer was then compared to the other 5 transducers using a customized power meter provided by Smith and Nephew Inc. All base line measurements were made without the polystyrene plate. The polystyrene plate was only added to measure changes in intensity due to its presence.

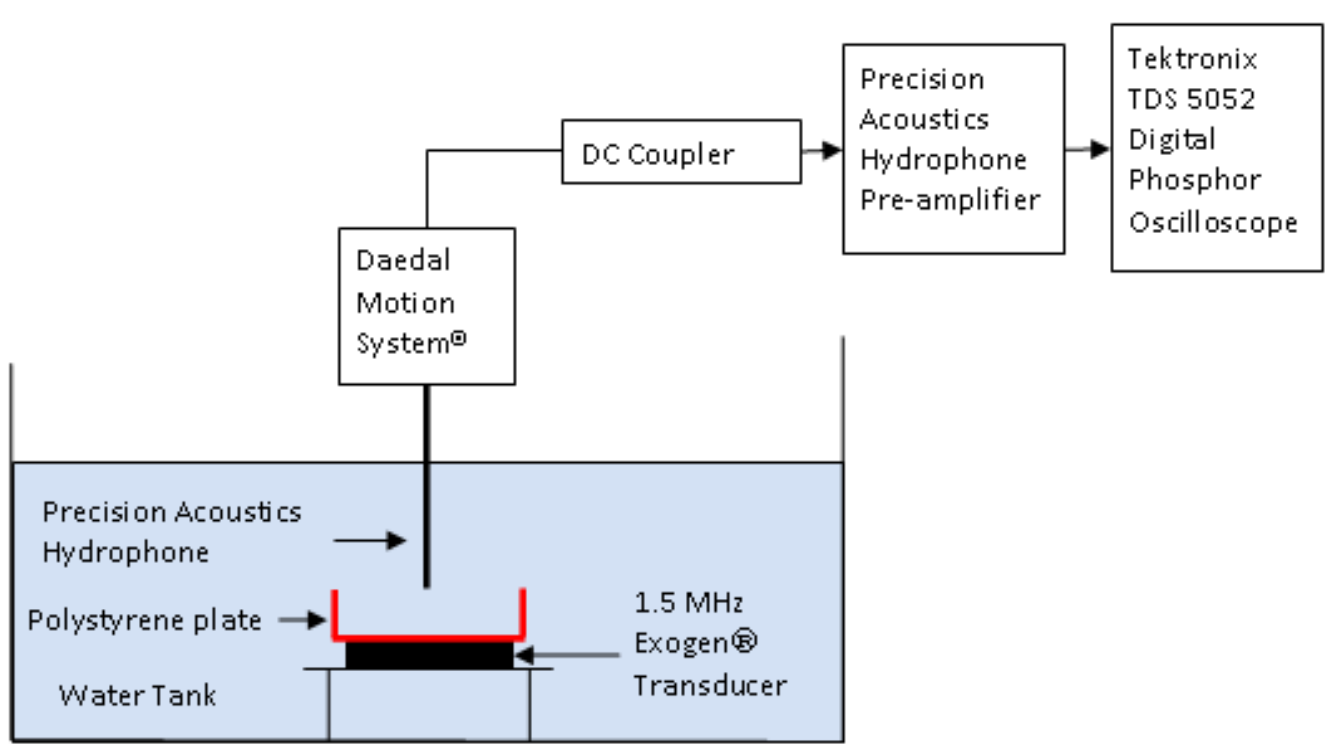

Figure 2-2: Acoustic output measurement set up using the calibrated hydrophone. 


\subsubsection{Calibrated Hydrophone}

All acoustic pressures measurements were done based on the method suggested in “Guidelines on Conducting Ultrasonic Intensity Measurements” (Hurrell 2007). Briefly, the guidelines suggest that the hydrophone measurements should be made in a water tank filled with degassed water. Once the hydrophone and transducer are aligned, the time domain wave form can be read off the oscilloscope and the frequency spectrum should be obtained to confirm the contribution of the central and harmonic frequencies. The voltage should then be converted to pressure using the chart in Figure 2-3, where voltage is then converted to pressure using sensitivity based on the frequency spectrum where:

$p=\frac{V}{M(f)}$

In equation 2.1, $p[\mathrm{MPa}]$ is the pressure, $V[\mathrm{mV}]$ is the voltage and $M(f)\left[\frac{\mathrm{mV}}{\mathrm{MPa}}\right]$ is the frequency dependent hydrophone sensitivity. If there are harmonic frequencies in addition to the central frequency, then each pressure is calculated individually based on frequency. The intensities are calculated and then summed. For this research the peak to peak voltage was read off the oscilloscope, excluding voltage spikes, and the peak voltage was used to calculate pressure. For a frequency of $1.5 \mathrm{MHz}$, the hydrophone sensitivity was $1.965 \mathrm{~V} / \mathrm{MPa}$. The active diameter of the hydrophone is $0.2 \mathrm{~mm}$. 


\begin{tabular}{|lc|}
\hline Frequency $(\mathbf{M H z})$ & Sensitivity $(\mathbf{m V} / \mathbf{M P a})$ \\
1.0 & 1682.4 \\
2.0 & 2260.5 \\
3.0 & 2775.9 \\
4.0 & 3211.5 \\
5.0 & 2694.0 \\
6.0 & 2805.5 \\
7.0 & 2292.4 \\
8.0 & 2102.2 \\
\hline
\end{tabular}

Figure 2-3: The calibrated hydrophone sensitivity table (Precision Acoustics 2007).

A 3-axes Daedal Motion System (Parker Hannifin Corp., Electromechanical Automation

Division of Daedal, Irwin, PA) was used to position the hydrophone. The system was operated manually. The positioning accuracy in all 3 axes (x, $y$, and $\mathrm{z}$ ) is $+/-0.5 \mathrm{~mm}$.

\subsubsection{Voltage measurements}

A Tektronix TDS 5052 digital oscilloscope was used to take peak to peak voltage. Once the peak voltage was located, axial voltage measurements were taken every $1 \mathrm{~mm}$ from 3-20 mm away from the face of the transducer along the acoustic axis. The measurements were taken at approximately 10 second intervals. Five measurements were taken at each $1 \mathrm{~mm}$ interval. The average of five measurements was used. Lateral voltage measurements were taken at 1, 2, and $13 \mathrm{~mm}$ away from the transducer face across the acoustic axis. The lateral voltage measurements were taken at $0.25 \mathrm{~mm}$ spacing. These measurements were used to confirm the effective radiating area of the transducer, and estimate the intensity in the radiating field. 


\subsubsection{Estimation of Spatial Average Temporal Average Intensity (IsATA)}

As described in chapter1, an effective method for assessing I IATA was to first find the pulsed average intensity $\left(\mathrm{I}_{\mathrm{PA}}\right)$ given by Preston (Preston 1991):

$I_{P A}=\frac{p_{0}^{2}}{2 \rho C}$

The $I_{P A}$ was converted into the $I_{T A}$ by multiplying by the duty cycle (see chapter 1 equation 1.8 for details). Therefore

$I_{T A}=I_{P A} \times D C$

Where Duty Cycle $(D C)$ is the pulse width divided by the pulse repetition period. Then, the

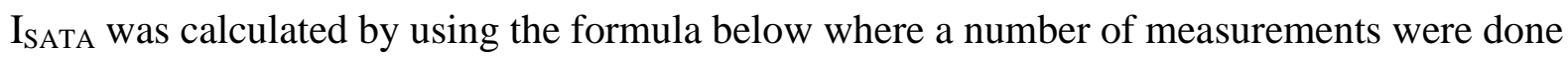
across the beam at a given depth:

$I_{S A T A}=\frac{\sum I_{T A}}{\text { total number of points }}$

By definition, $\mathrm{I}_{\mathrm{SATA}}$ include all points where $\mathrm{I}_{\mathrm{TA}}>0.25 \mathrm{I}_{\mathrm{TA}}$ Max (Figure 2-4). The cutoff of $-6 \mathrm{~dB}$ is where intensity drops below $25 \%$. In practice averaging all of the time averaged intensities should be approximately equal to $\mathrm{I}_{\mathrm{SATA}}$ (Preston 1991, Hurrell 2007). 


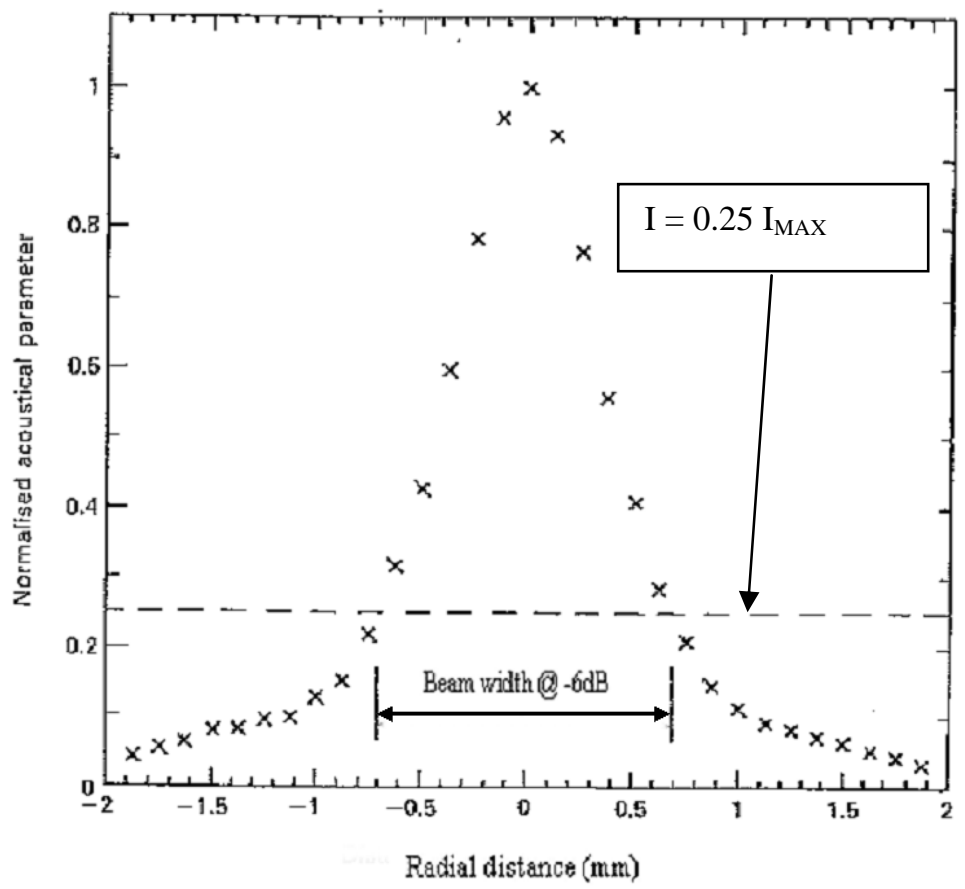

Figure 2-4: Cut off intensity for calculating active intensity and the effective radiating area (Preston 1991).

\subsubsection{Estimation of Frequency, Pulse Duration, Pulse repetition, Duty Cycle}

The data was gathered by capturing the peak to peak voltage during a full cycle of the ultrasound signal with a $1 \mathrm{kHz}$ PRF and $200 \mu$ s pulse width. Multiple single captures of oscilloscope screen used to confirm frequency, pulse duration, pulse repetition frequency and duty cycle.

\subsubsection{Effect of polystyrene cell culture plate on acoustic intensity}

To measure the effect of the polystyrene plate on intensity a polystyrene cell culture plate was placed in front of the transducer. The axial voltage measurements were repeated following the steps described above. The area of interest was approximately $13 \mathrm{~mm}$ away from the face of the transducer. This distance away from the transducer is the area of activity for cells and media. 


\subsubsection{Calculation of Intensity Lose due to Polystyrene Cell Plate}

To calculate the intensity loss due to the polystyrene plate; the geometry in Figure 2-5 was considered. The total amount of energy that reached the region of interest was the attenuated intensity that had been transmitted through the polystyrene plate.

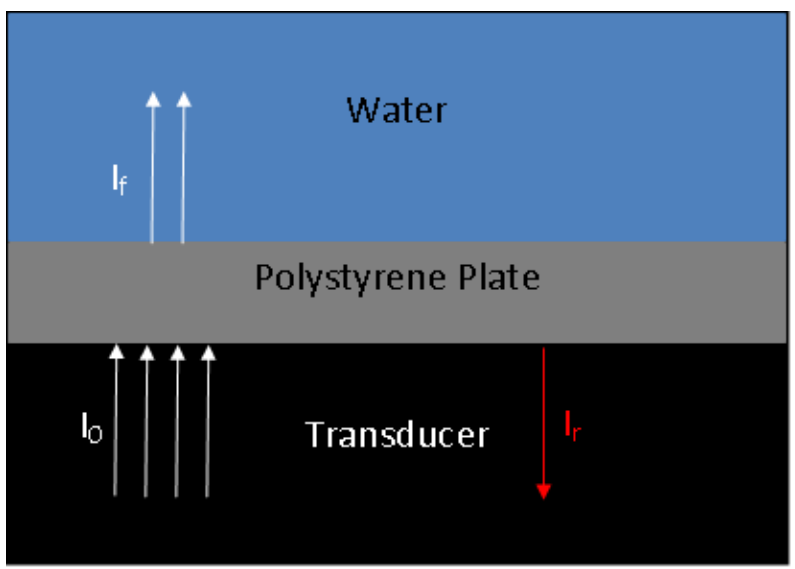

Figure 2-5: Schematic of intensity loss of at the water polystyrene interface. $I_{0}$ is the initial intensity incident on the transducer surface. $I_{f}$ indicates intensity remaining after attenuation, while $I_{r}$ indicates loss due to reflection.

According to Waters et al. (Waters et al 2003) frequency dependant attenuation, through a polystyrene plate, can be calculated with the following experimentally derived formula:

$\alpha(f)=0.133+0.0232 f^{1.26} \frac{\mathrm{Np}}{\mathrm{cm} \mathrm{MHz}}$ 


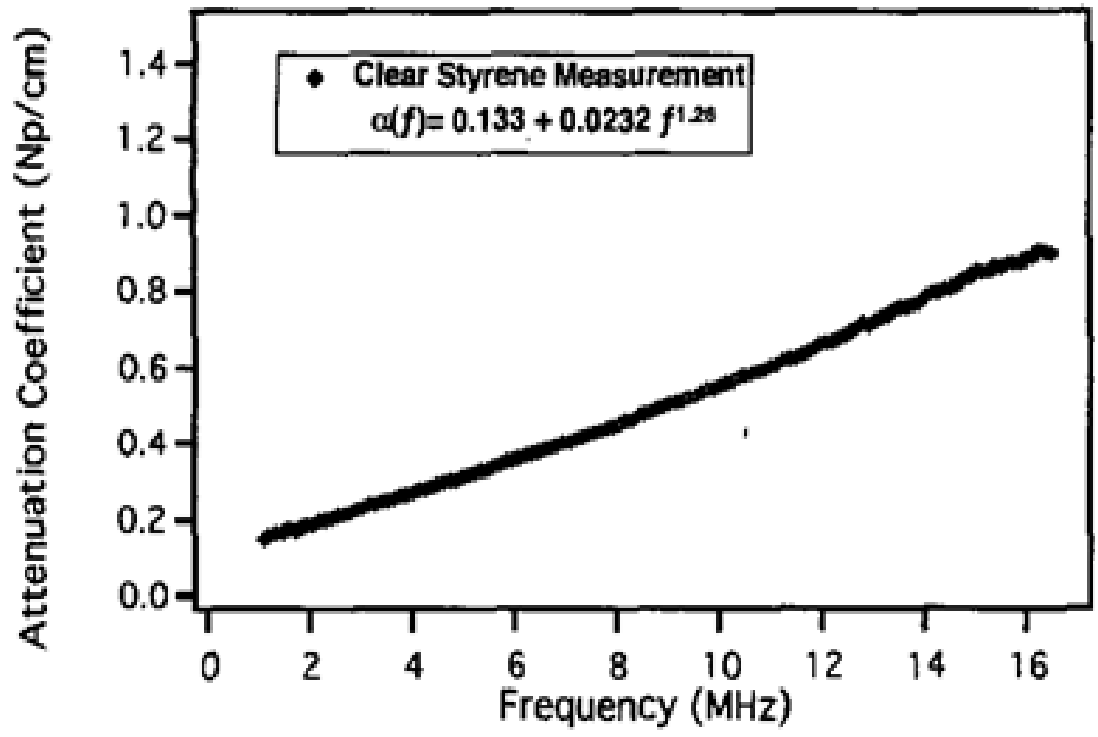

Figure 2-6: Frequency dependant attenuation of ultrasound through polystyrene (Waters et al 2003).

Figure 2-6 indicates a log-linear relationship between attenuation and frequency of polystyrene. This relationship and equation 2.5 were used to calculate the theoretical loss of intensity of an ultrasound wave as it passed through the polystyrene plate. The attenuation of the polystyrene plate would be:

$\alpha(1.5)=0.133+0.0232(1.5)^{1.26}=0.172 \frac{\mathrm{Np}}{\mathrm{cm}}=1.49 \frac{\mathrm{dB}}{\mathrm{cm}} @ 1.5 \mathrm{MHz} \quad$ (See equation 2.5) In the theoretical set up the ultrasound wave would need to travel through $1 \mathrm{~mm}$ of polystyrene and then travel through $13 \mathrm{~mm}$ of water before getting to the region of interest.

Loss of intensity due to attenuation can be described as (Cobbold 2007):

$I(x)=I(0) e^{(-2 \alpha x)}$

where $\alpha_{\text {polystyrene }}=1.49 \mathrm{~dB} / \mathrm{cm} @ 1.5 \mathrm{MHz}$ assuming an initial intensity of $30 \mathrm{~mW} / \mathrm{cm}^{2}$ (as per manufacturer specifications). 
At the interface between the transducer and the polystyrene plate, a portion of the intensity will be reflected back towards the transducer with an intensity reflection coefficient given by (Cobbold 2007):

$R_{I}=\left(\frac{Z_{2} \cos \theta_{i}-Z_{1} \cos \theta_{t}}{Z_{2} \cos \theta_{i}+Z_{1} \cos \theta_{t}}\right)^{2}$

$\mathrm{R}_{\mathrm{I}}$ is the intensity reflection coefficient, $\theta_{\mathrm{i}}$ and $\theta_{\mathrm{t}}$ are the incident and transmitted angles of the wave and $\mathrm{Z}$ is the acoustic impedance (see chapter 1 equation 1.4 for further details). If the acoustic impedance of polystyrene is assumed as 2.42 MRayls (Selfridge 2009) and the acoustic impedance of water is assumed as 1.48 MRayls, and the incident angle is assumed $=0$, then:

$R_{I}=\left(\frac{2.42-1.48}{2.42+1.48}\right)^{2}=0.058$

(See equation 2.7)

In this case the reflected intensity $\left(I_{R}\right)$ can be calculated as a portion of the incident intensity $\left(I_{I}\right)$ :

$I_{R=} I_{I} \times R_{I}=30 * 0.058=1.74 \frac{\mathrm{mW}}{\mathrm{cm}^{2}}$

The total intensity $\left(I_{T}\right)$ remaining to be transmitted through the polystyrene is then:

$I_{T=} I_{I}-I_{R}=30-1.74=28.26 \frac{\mathrm{mW}}{\mathrm{cm}^{2}}$

The intensity is then attenuated as it passes through the layer of $1 \mathrm{~mm}$ thick polystyrene plate, as:

$I(0.1 \mathrm{~cm})=28.26 e^{(-2 \times 1.49 \times 0.1)}=20.98 \frac{\mathrm{mW}}{\mathrm{cm}^{2}}$

(See equation 2.6)

From the above calculations about $1 / 3$ of the initial intensity was lost by the time it reaches the region of interest. In addition, the manufacturer gives the intensity range as 30 $\mathrm{mW} / \mathrm{cm}^{2} \pm 30 \%$. This means the possible variation of intensity in the region of interest could range between 14.30 to $27.66 \mathrm{~mW} / \mathrm{cm}^{2}$. 


\subsubsection{Exogen ${ }^{\circledR}$ Device Acoustic Beam Simulation}

The ultrasound beam generated by the Exogen ${ }^{\circledR}$ device was simulated in Matlab ${ }^{\circledR}$ using an in-house ultrasound field simulation program (written by J. Tavakkoli and updated by M. Kusha, Dept. of Physics, Ryerson University, 2008). The ultrasound field simulation was used to confirm measured intensity values. Figure 2-7 illustrates the geometry used to simulate pressure in the ultrasound field.

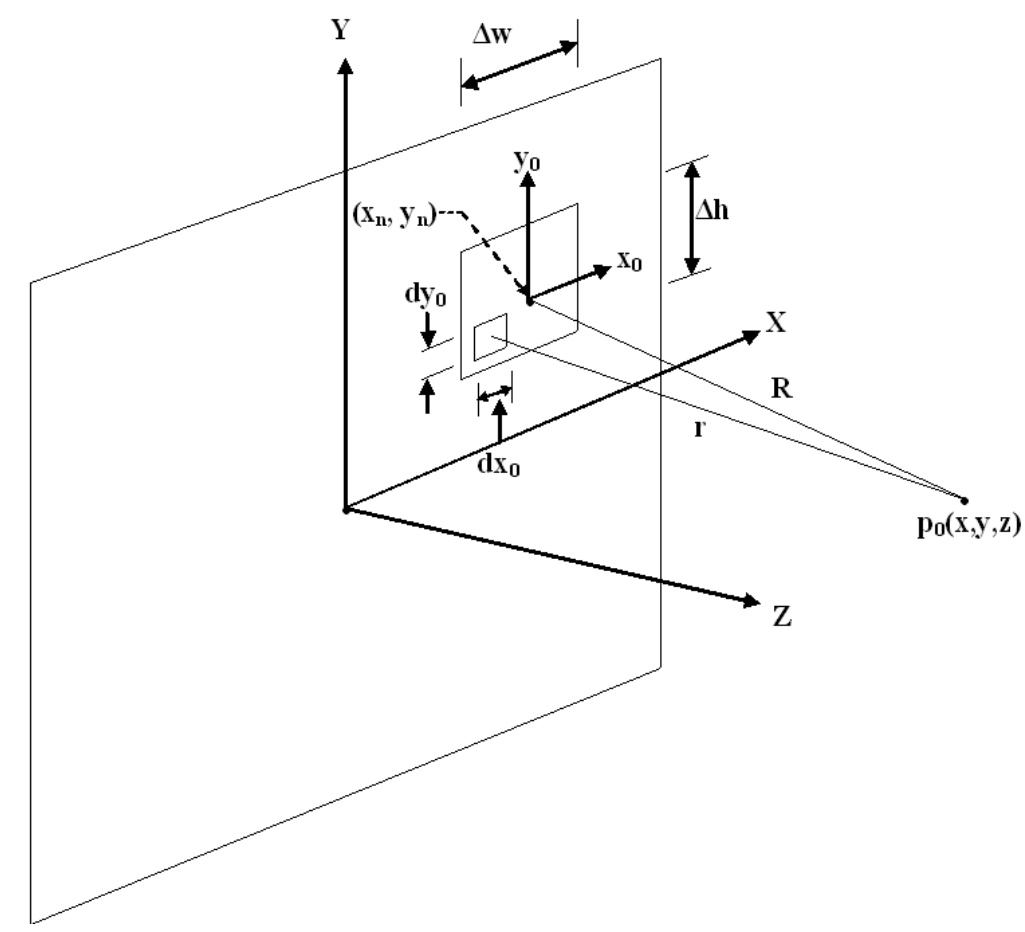

Figure 2-7: Coordinate system and geometry used to define pressure at any point in the ultrasound field.

The program simulates a continuous ultrasound waves in a homogenous medium, where the ultrasound source is divided into small rectangular elements. The pressure at any point in the ultrasound field is then calculated by summing up the pressure contribution of each rectangular element over the entire pressure field. The contribution of each surface element is calculated 
through an analytical formula originally proposed by Ocheltree and Frizzel (Ocheltree and Frizzell 1989):

$p_{0}=\frac{j \rho_{0} c_{0} \Delta w \Delta h}{\lambda} \frac{v_{n o} \exp (-(\alpha+j k) R}{R} \operatorname{sinc}\left(\frac{k x^{\prime} \Delta w}{2 R}\right) \operatorname{sinc}\left(\frac{k y^{\prime} \Delta h}{2 R}\right)$

Figure 2-7 illustrates the coordianate system and geometry of an individual element to pressure in space. According to the geomentry, Equation 2.4 can be described, where $p_{0}$ is any point in the ultrasound field, $\rho_{0}$ density, $\mathrm{c}_{0}$ speed of sound (phase velocity of a sound wave), $\Delta w$ and $\Delta h$ are the width and height elements of the transducer, $\lambda$ is the wavelength, $v_{\text {no }}$ complex surface element velocity, $\alpha$ is the medium attenuation, $\mathrm{k}$ is the wave number, $R$ is the distance from center of the element to $p_{0}$, while $\mathrm{r}$ is the distance from the grid space to $p_{0}, x^{\prime}=x-x_{n}$ or the $\mathrm{x}$ distance from the center of the element, and $y^{\prime}=y-y_{n}$ or the $\mathrm{x}$ distance from the center of the element.

The ultrasound field simulation program uses the pressure obtained from equation 2.4 to calculate $\mathrm{I}_{\mathrm{TA}}$ for a continuous wave at discrete points. For simulating the Exogen ${ }^{\circledR}$ ultrasound wave, the discrete time averaged intensities are considered pulsed average. To convert these values to model a pulsed wave form the time averaged intensities are multiplied by the duty cycle.

The simulation parameters were set with a transducer diameter of $22.2 \mathrm{~mm}$, an element size of $0.25 \mathrm{~mm}$ and a step size of $0.1 \mathrm{~mm}$. The simulation was run for a calculation domain of 0-250 mm axially from the surface of the transducer, and 0-5 mm radially from the transducer acoustic axis. 


\subsection{Results}

\subsubsection{Estimation of Acoustic Intensity}

For this study, the near field of the Exogen ${ }^{\circledR}$ device was used, which reflects most clinical applications. For this reason, the measurements were only taken in the near field. These measurements were based on 5 voltage measurements ranging from 120-123 mV. The time averaged intensities ranged from $23-24 \mathrm{~mW} / \mathrm{cm}^{2}$. Using measurements taken with the calibrated hydrophone, the $\mathrm{I}_{\mathrm{TA}}$ at $13 \mathrm{~mm}$ was found to be $23 \mathrm{~mW} / \mathrm{cm}^{2}$.

In Figure 2-8 there are variations in the measurements as the hydrophone was scanned laterally at a distance of $13 \mathrm{~mm}$ from the transducer surface. Using a cut off value of $-6 \mathrm{~dB}$, as suggested in Preston (Preston 1991), I IATA was calculated by averaging all the points above I > $0.25 \mathrm{I}_{\text {Max }}$. The intensity cut off $13 \mathrm{~mm}$ was obtained at $\mathrm{I}_{\mathrm{TA}}=10 \mathrm{~mW} / \mathrm{cm}^{2}$. $\mathrm{I}_{\mathrm{SATA}}$ at this axial distance was found to be $24 \mathrm{~mW} / \mathrm{cm}^{2}$.

The axial intensity profile, measured in the near field of the transducer, showed variation associated with this region (Figure 2-9). The time averaged intensity varied from 11 to 28 $\mathrm{mW} / \mathrm{cm}^{2}$ along the acoustic axis of the transducer from 3 to $20 \mathrm{~mm}$. 


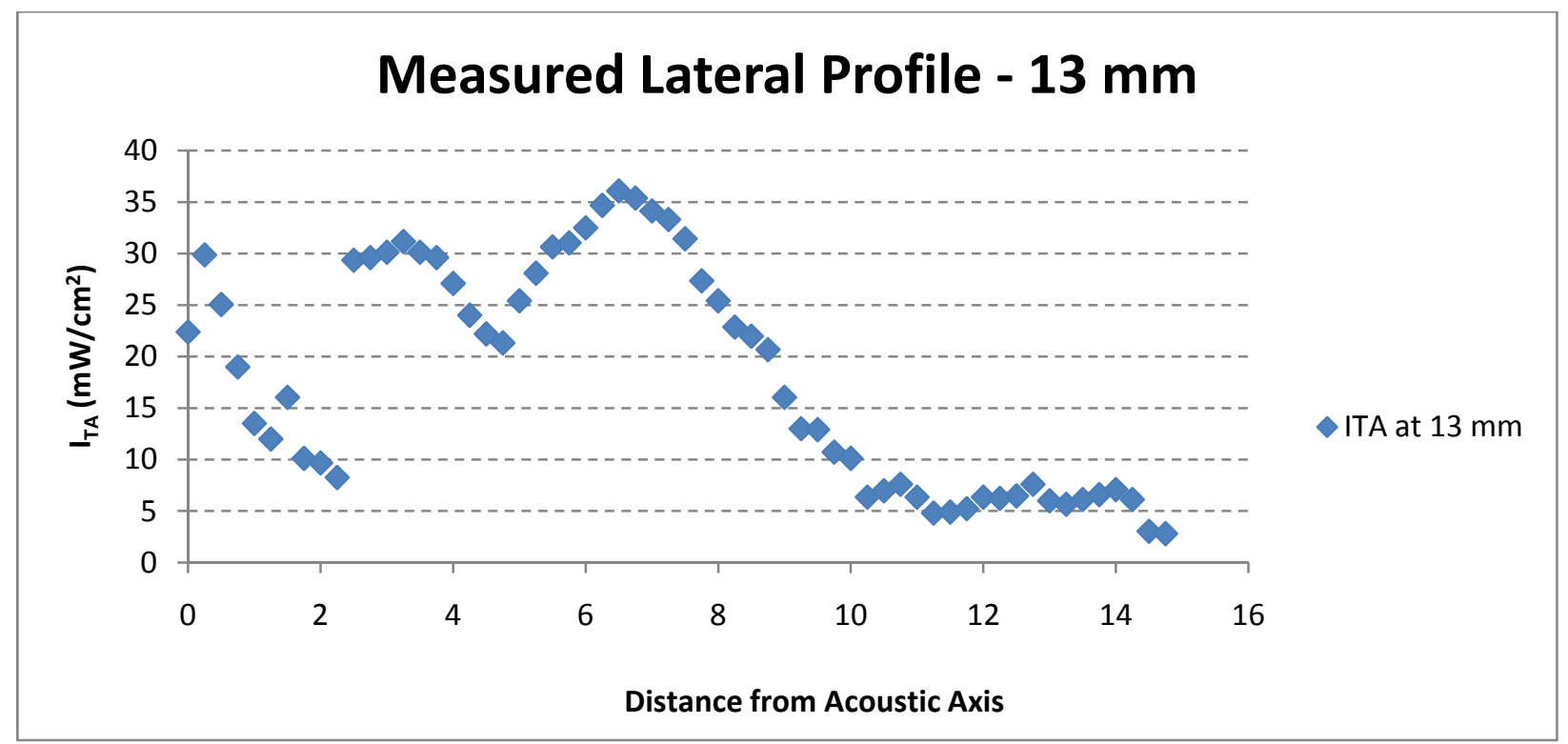

Figure 2-8: Measured Lateral Intensity Profile.

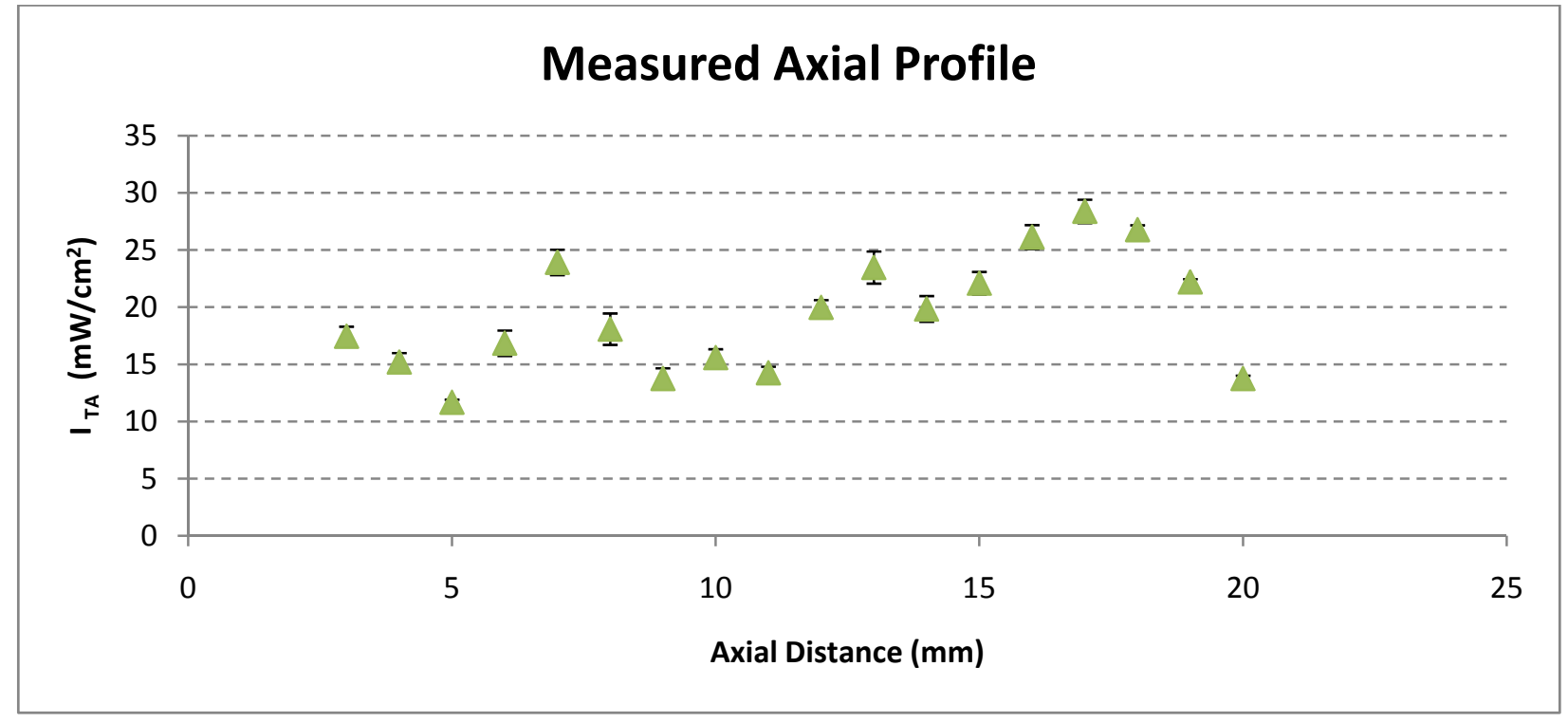

Figure 2-9: Measured Axial Intensity Profile. 


\subsubsection{Effective Radiating Area}

The effective radiating area should be smaller than the actual radius of the transducer crystal. For purposes of this thesis ERA was estimated as the point at which the intensity drops below 25\% (or -6dB) of its maximum value (Figure 2-4) (Preston 1991).

To reduce the variation and assess the ERA, the log of the intensity profile was taken. Although there were some fluctuations in the intensity measurements over the face of the transducer Figure 2-10 suggests that there was a distinct drop off of the intensity beyond $12 \mathrm{~mm}$. The intensity dropped below $25 \%$ of the peak intensity at a radius of $12.25 \mathrm{~mm}$ at $1 \mathrm{~mm}$ away from the transducer surface. This seems reasonable since the measurement falls between, the manufacturer stated radius of $11.1 \mathrm{~mm}$ and the transducer housing radius of $13.5 \mathrm{~mm}$. The manufacturer states the ERA as $3.88 \mathrm{~cm}^{2}$, then the comparable measured ERA would be 4.71 $\mathrm{cm}^{2}$.

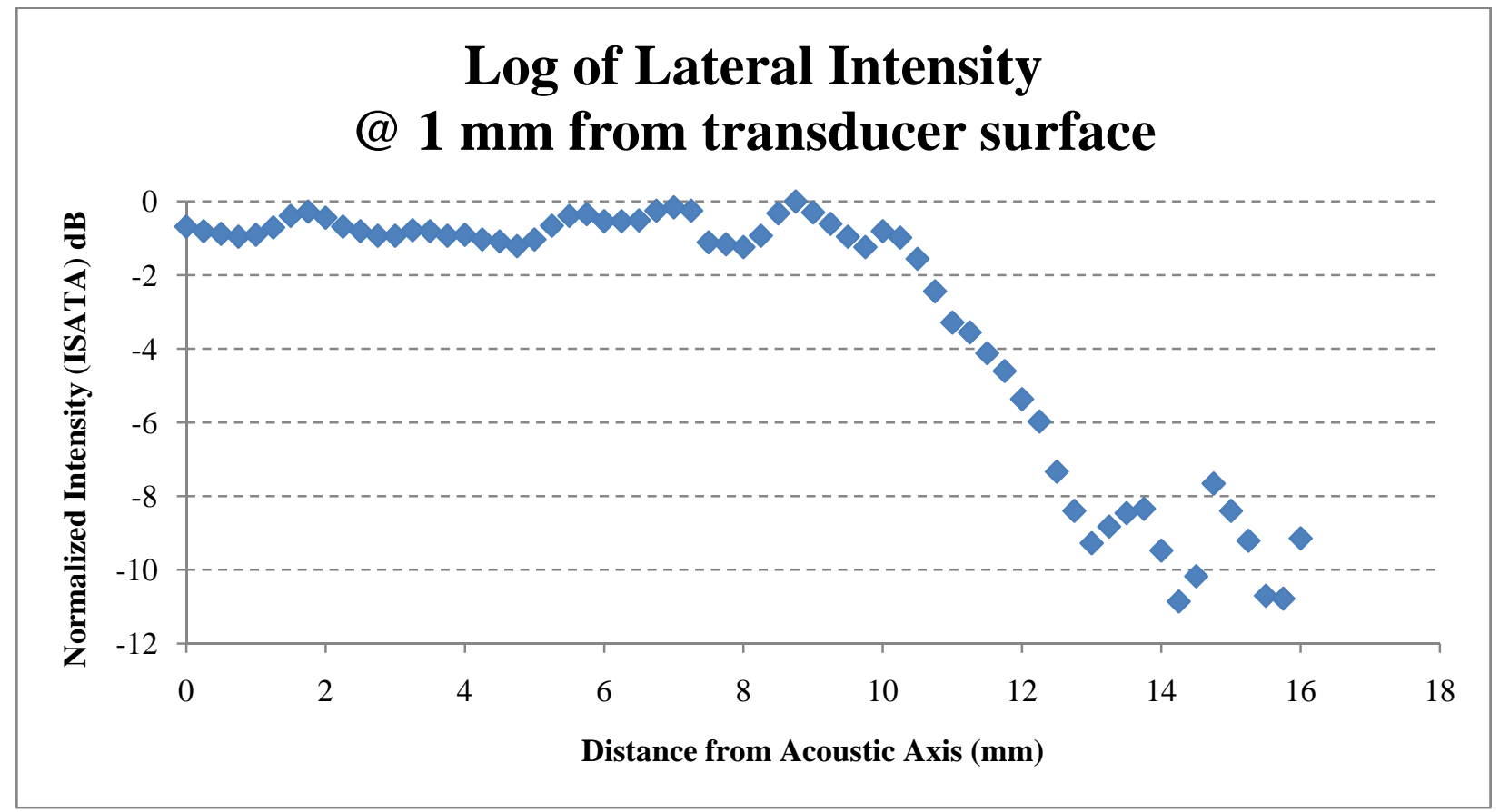

Figure 2-10: Lateral Intensity (log scale). 


\subsubsection{Estimation of Pulse Frequency, Pulse Duration, Pulse Repetition Frequency (PRF), and Duty Cycle (DC)}

All measurements in this section were taken at $13 \mathrm{~mm}$ away from the face of the transducer. This was the area of interest because the cells being treated would be located approximately at this distance. Table 2-1 gives a comparative summary of all findings.

The average of the five measurements of the signal's center frequency was $1.50 \mathrm{MHz}$ $\pm 0.2 \%$, where Figure 2-11is a sample measurement. The Pulse Duration was measured at $200 \mu \mathrm{s}$ for each of the 5 samples. All samples seem to have a distinct tail (Figure 2-11). The tail's amplitude is approximately $20 \%$ of the peak amplitude and is approximately $200 \mu$ s long. Due to the small amplitude of the tail, the effect on intensity would likely be less than $5 \%$. The source of the tail pulse is not clear; however it could originate from internal reflections between the crystal and the matching layer, or possible issues with the driving electronics.

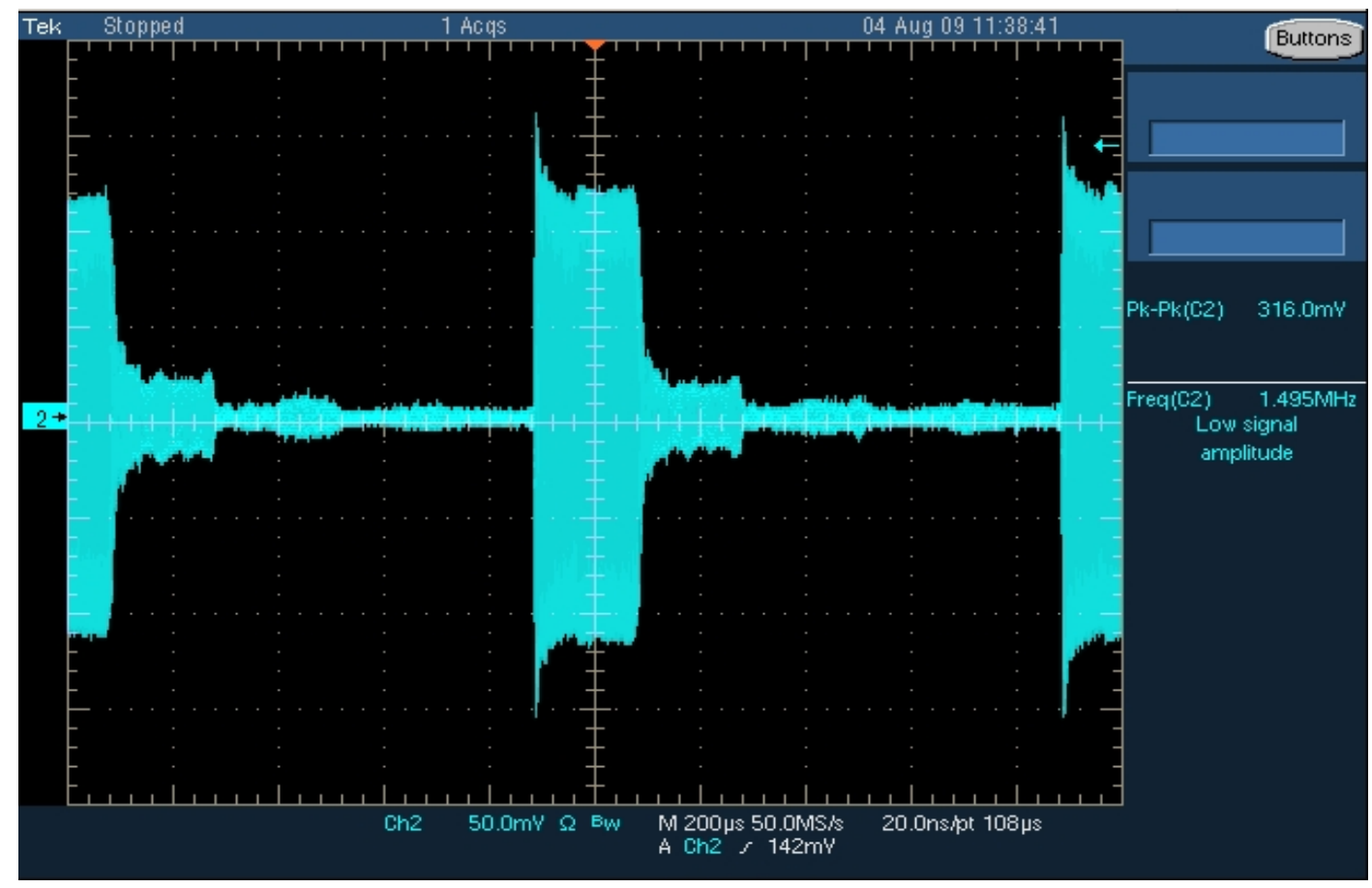

Figure 2-11: Hydrophone signal at $\mathbf{1 3 m m}$ away from transducer face. 
The PRF was measured as $1 \mathrm{KHz}$ and the DC was $20 \%$ with no measureable errors. All of the measured characteristics aside from ERA were within the manufacturer specifications. The ERA affects the intensity, therefore as the ERA increases the $\mathrm{I}_{\mathrm{SATA}}$ decreases by the same ratio.

\begin{tabular}{ccc}
\hline \hline & Manufacturer Characteristics & Measured Characteristics \\
\hline Pulse Central Frequency & $1.5 \mathrm{MHz} \pm 5 \%$ & $1.50 \mathrm{MHz} \pm 0.2 \%$ \\
Pulse Duration & $200 \mu \mathrm{s} \pm 10 \%$ & $200 \mu \mathrm{s}$ \\
PRF & $1 \mathrm{KHz} \pm 1 \%$ & $1 \mathrm{KHz}$ \\
Duty Cycle & $20 \%$ & $20 \%$ \\
Effective Radiating area & $3.88 \mathrm{~cm}^{2} \pm 1 \%$ & $4.71 \mathrm{~cm}^{2}$ \\
ISATA (13 mm) & $30 \pm 30 \% \mathrm{~mW} / \mathrm{cm}^{2}$ & $24 \mathrm{~mW} / \mathrm{cm}^{2}$
\end{tabular}

Table 2-1: Comparison of Exogen ${ }^{\circledR}$ Device Manufacturer and Measured Characteristics.

\subsubsection{Polystyrene Plate Model}

The effect of the $1 \mathrm{~mm}$ thick polystyrene plate on the ultrasound intensity was investigated. The polystyrene plate was place against the transducer face. Measurements in Figure 2-12 show that there was a reduction in the intensity at the area of interest $13 \mathrm{~mm}$ away from the transducer face. At this distance the intensity was reduced from $23 \pm 3 \mathrm{~mW} / \mathrm{cm}^{2}$ to 10 $\pm 0.5 \mathrm{~mW} / \mathrm{cm}^{2}$ when the polystyrene plate was placed in front of the transducer; more than a $50 \%$ reduction in intensity. 


\section{Axial Intensity comparison with and without polystyrene cell plate}

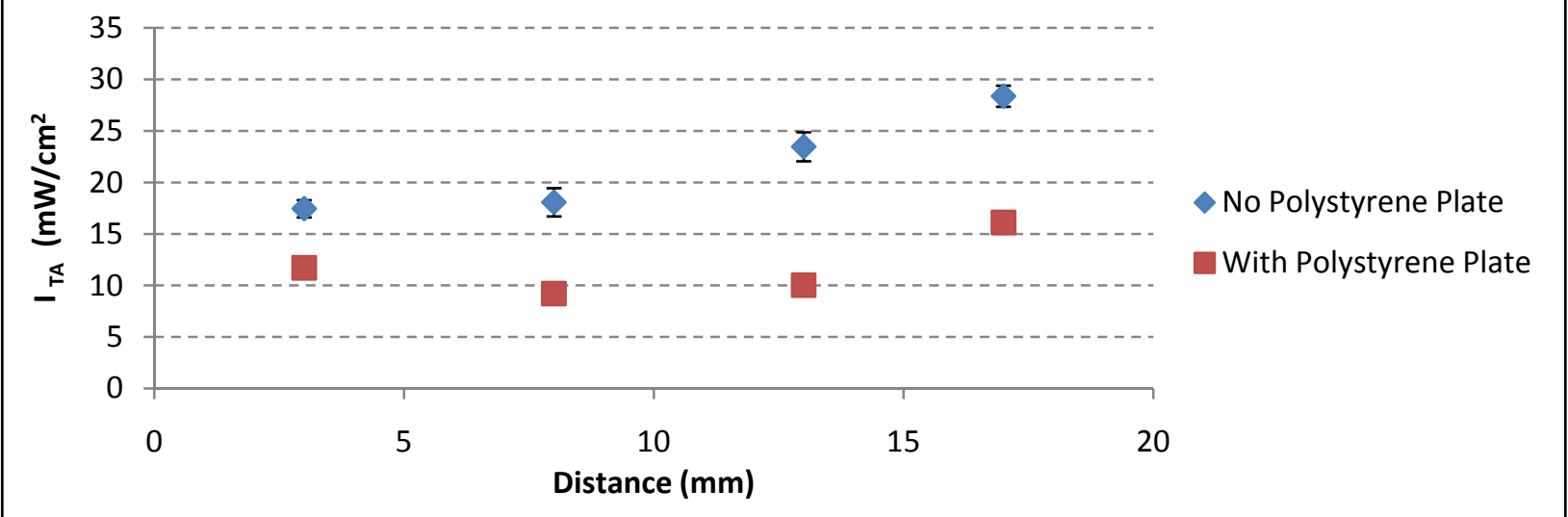

Figure 2-12: Intensity comparison with and without polystyrene cell plate. For measured intensities with and without the polystyrene plate, the plate was placed against the transducer.

\subsubsection{Computer Simulations Results - Intensity Simulation Results}

The simulation of the Exogen ${ }^{\circledR}$ transducer’s axial intensity followed the expected pattern of a flat circular transducer (Cobbold 2007) with the Rayleigh distance at 120mm (Figure 2-13). Accuracy of the simulation was directly dependent on the element grid and calculation step sizes. To maximize the accuracy of the axial intensity, the element grid was set to $0.25 \mathrm{~mm}$. With this element grid size there was only a 20\% difference in intensity peak amplitudes along the transducer’s acoustic axis. In addition, based on linear acoustic theory in a plane transducer, when the ratio between axial distance and transducer diameter is above 2, the relationship between pressure and intensity is accurate (Cobbold 2007). 


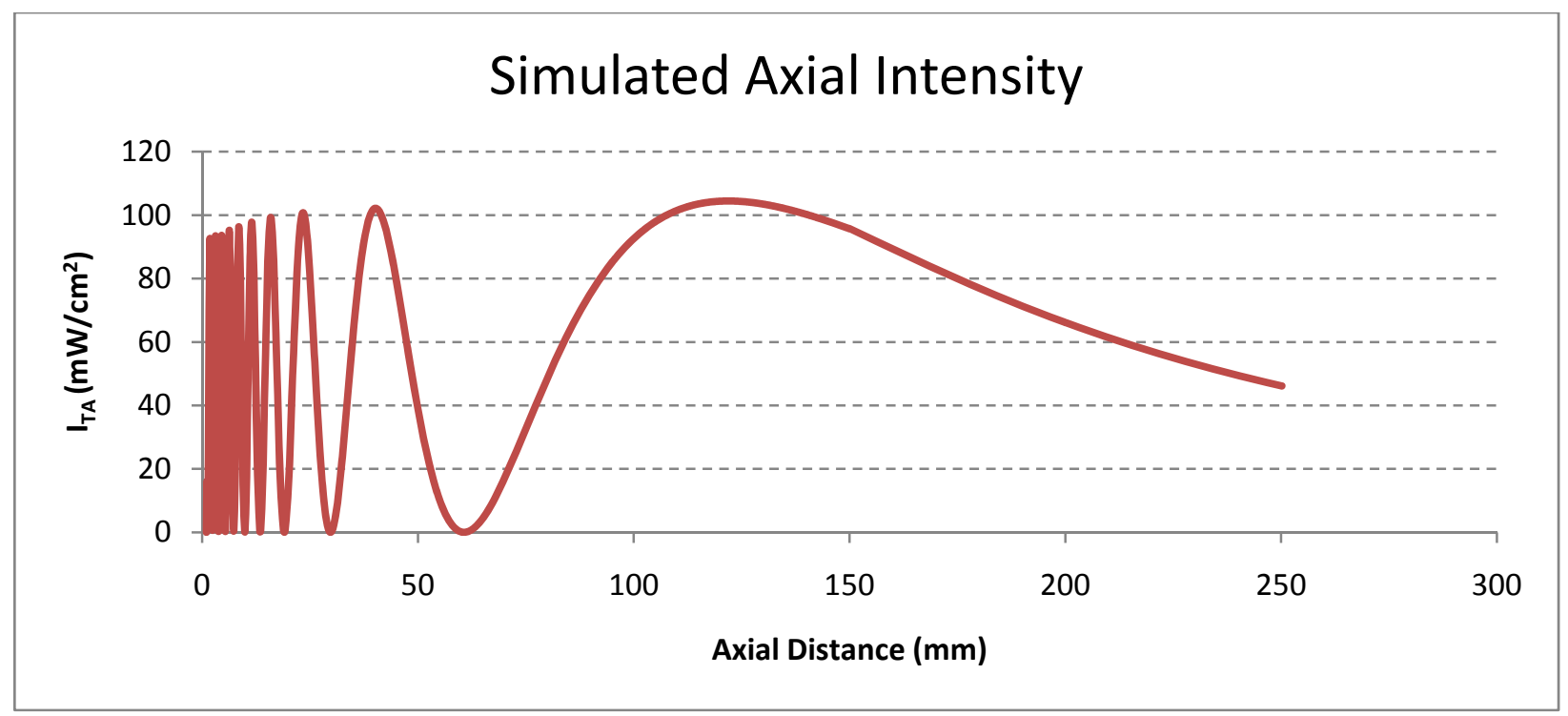

Figure 2-13: Simulated Axial Intensity.

At $13 \mathrm{~mm}$ from the transducer surface the simulated and measured $\mathrm{I}_{\mathrm{SATA}}$ were within 2 $\mathrm{mW} / \mathrm{cm}^{2}$ of each other (Error! Reference source not found. and Figure 2-14). The simulated $\mathrm{I}_{\mathrm{SATA}}=26 \mathrm{~mW} / \mathrm{cm}^{2}$ while the measured $\mathrm{I}_{\mathrm{SATA}}=24 \mathrm{~mW} / \mathrm{cm}$

The step size used in these simulations was $0.1 \mathrm{~mm}$. The active area of the hydrophone used in measurements is $0.2 \mathrm{~mm}$. This means that the intensity values obtained in simulations and measurements could not be directly compared. To compare simulated and measured values of intensity the simulated $\mathrm{I}_{\mathrm{TA}}$ was converted into $\mathrm{I}_{\mathrm{SATA}}$. The simulated $\mathrm{I}_{\mathrm{SATA}}$ was calculated in a method similar to the one given by Preston (Preston 1991) (see equation 1.17 in chapter 1 for more details). The instantaneous intensities, calculated at a given distance from the transducer's surface, were then averaged over the lateral beam profile at that distance. The transducer's lateral beam profile has a multi ring structure (Figure 2-15). The $I_{T A}$ was averaged over discrete rings of $0.1 \mathrm{~mm}$ in thickness, which corresponds to the surface of the hydrophone active element. The $I_{T A}$ measurements could then be compared to individual hydrophone measurements. In theory this is possible, however, due to the limitations of the positioning system used for this experiment, 
these comparisons were not practical. For a better comparison, these simulated $I_{T A}$ values are then averaged over the surface of the surface of the transducer. The average the simulated $I_{T A}$ is $I_{S A T A}$. In this ways the simulated and measured spatially averaged temporally averaged intensities can be compared.

$I_{T A}=\frac{\sum I_{T A} A_{\text {ring }}}{\sum A_{\text {ring }}}$

$I_{S A T A}=\frac{\sum I_{T A} A}{\sum A}$

The measured and simulated axial intensities at between 12 and $19 \mathrm{~mm}$ away from the transducer face has a maximum difference of $10 \mathrm{~mW} / \mathrm{cm}^{2}$ and a difference of $5 \mathrm{~mW} / \mathrm{cm}^{2}$ in the region of interest (Figure 2-14). The difference between the measured and simulated values can be accounted for by the possibility of small inaccuracies in the positioning system. In the nearfield of the transducer the intensity can have large variations. Small errors in the positioning system can lead to large errors in the intensity measurement. It is possible that the measured peak or valley may not coincide with the simulated peak or valley.

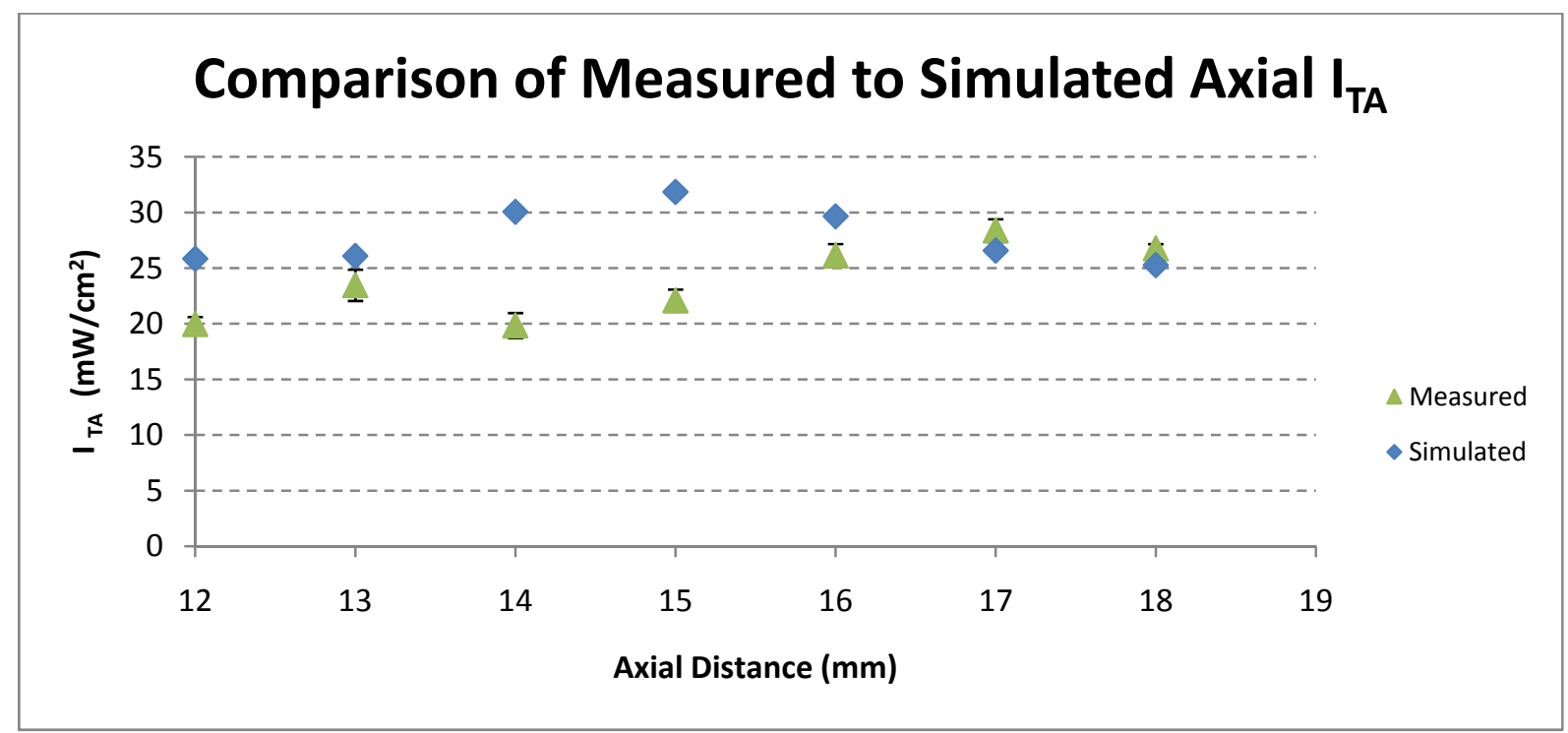

Figure 2-14: Simulated vs. Measured Axial intensity 11-20 mm. 
A comparison was also be made of the lateral simulated and measured lateral intensities.

The lateral slices across the acoustic axis around the area of interest show that there was significant variation in the lateral beam profiles as the axial distance changes in the near field. $\mathrm{I}_{\mathrm{SATA}}=26.5-28.5 \mathrm{~mW} / \mathrm{cm}^{2}$ between $11-15 \mathrm{~mm}$.

As can be seen in Figure 2-16 the profiles and maximum intensities vary in location as well. When the measured lateral profiles are plotted against a range of simulate intensities, the measured intensities are in the same range as the simulated intensities (Figure 2-16).
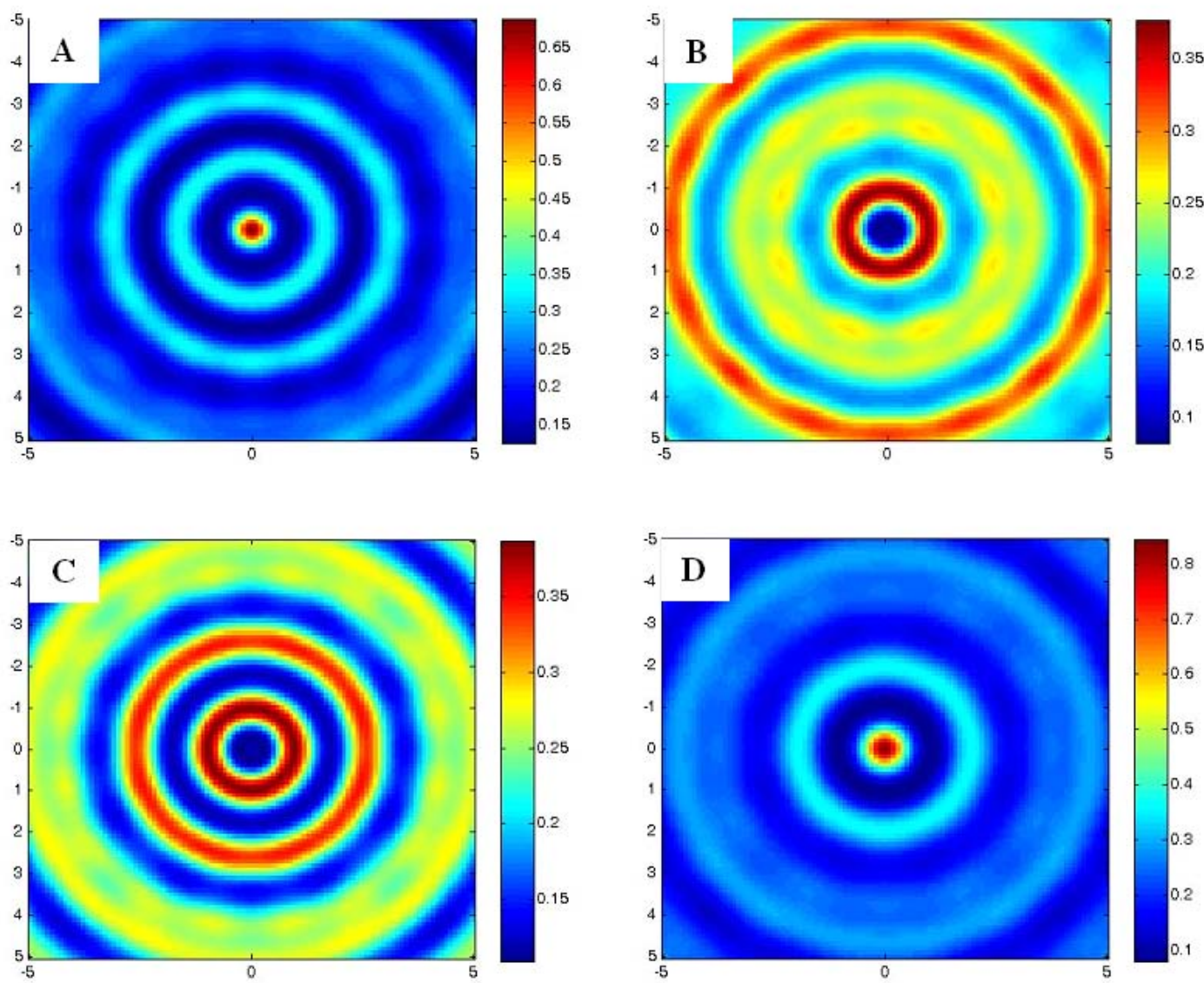

Figure 2-15: Lateral intensity profiles where the intensity is averaged over $1 \mathrm{~mm}$ diameter thick rings A) 11 mm, B) $12 \mathrm{~mm}$, C) $13 \mathrm{~mm}$, D) $15 \mathrm{~mm}$. 


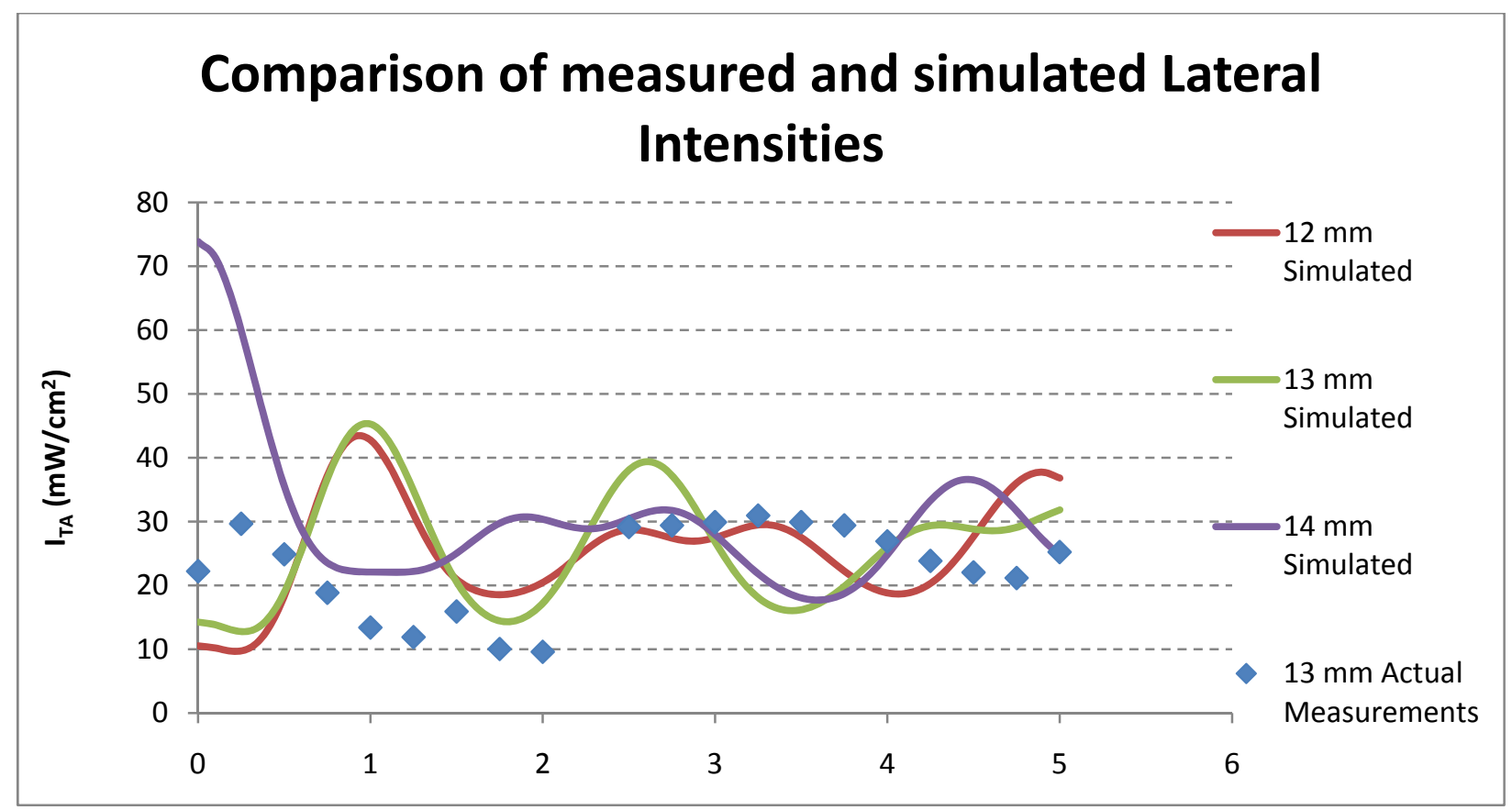

Figure 2-16: Simulated Lateral Intensity vs. Measured Intensities.

\subsection{Discussion and Conclusion}

The purpose of this chapter was to independently measure the acoustic output characteristics of the Exogen ${ }^{\circledR}$ research transducer. The main characteristics investigated were: I $_{\mathrm{SATA}}$, center frequency, pulse width, pulse repetition frequency, duty cycle, and effective radiating area (ERA). With the exception of the effective radiating area, all other measured values were found to be within the manufacturer stated characteristics.

The measured effective radiating area does not fall within the manufacturer stated characteristics. According to Straub et al. (Straub et al 2008), the measurement of ERA is difficult to evaluate consistently due to a complicated dependence on beam geometry.

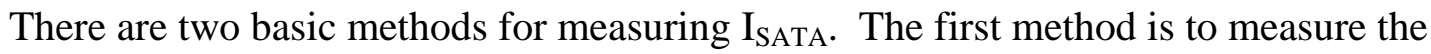
temporal average power with a radiating force balance, and then divide it by the effective radiating area. Acevedo et al. suggests that this is the most accurate method (Acevedo and Das- 
Gupta 2002). A more common method that was used in this thesis was to take several hydrophone measurements over the surface of the transducer and take the average of the measured point intensities over the number of points taken (Acevedo and Das-Gupta 2002). Measurements using the second method were found to be within manufacturing standards.

The effect of the polystyrene cell plate on intensity measurements was found to be significant. Although these cell culture plates are frequently used with various in vitro ultrasound studies, the intensity reducing effect of the plate has been consistently ignored. The manufacturer stated variation in the transducer intensity is $30 \%$. This variation in transducer intensity can theoretically lead to potential loss of intensity, due to a polystyrene plate, of $10 \%$ $50 \%$ depending on the true output intensity at the surface of the transducer. These measured results have shown intensity drops by of $58 \%$ of its initial value when the polystyrene plate is introduced between the transducer and the cell culture. For this reason, the intensity in the region of interest will be considered $\mathrm{I}_{\mathrm{SATA}}(13 \mathrm{~mm})=10 \pm 0.5 \mathrm{~mW} / \mathrm{cm}^{2}$. The cell culture experiments conducted in this thesis will have $\mathrm{I}_{\mathrm{SATA}}(13 \mathrm{~mm})=10 \mathrm{~mW} / \mathrm{cm}^{2}$ deposited onto the cells. 


\section{Chapter 3 Cell Culture Experiments: Materials and}

\section{Methods}

\subsection{Cell Culture}

MC3T3-E1 Sub clone 14 cells were obtained from ATCC Global Bioresource Center, Manassas, VA (Cat. No. CRL-2594 ${ }^{\mathrm{TM}}$ ). The MC3T3-E1 cells were mouse derived immortalized adherent calvarial preosteoblast cells. The sub clone was selected for high differentiation and mineralization after growth in media containing Ascorbic Acid (AA) and 3 to $4 \mathrm{mM}$ inorganic phosphate (Wang et al 1999). After approximately 10 days in culture these cells should form a well mineralized collagen matrix. These cells are good models for studying in vitro osteoblast differentiation (Wang et al 1999). They have behavior similar to primary calvarial osteoblasts (Wang et al 1999).

To maintain exponential growth cells were split so that the number of cells does not exceed the capacity of the container. Each splitting of the cells is called a passage. For this experiment the cells were passaged 3-4 days to maintain growth. Immediately after purchase the cells were split and passaged approximately 10 times and then refrozen for later use. All of the experiments done in this work have used cells that have been re-frozen. The cells were passaged less than 10 times after thawing.

\subsection{Cell Culture Equipment}

The basic equipment needed for culturing cells was, a water bath set at $37^{\circ} \mathrm{C}$ to warm up media, and an incubator to keep the cells at a consistent temperature of $37^{\circ} \mathrm{C}$ and at a consistent level 5\% $\mathrm{CO}_{2}$. Seventy percent ethanol was used to keep all surfaces free of contamination. 
Sterile pipettes and micro-pipettes, well culture plates and flasks, and media flasks, were used when in contact with cells or cell products.

\subsection{Preparation of Work Place}

All media was warmed up in a water bath for 15 minutes, to a nominal temperature in the water-bath to $37^{\circ} \mathrm{C}$ prior to use. The laminar flow hood was wiped down with $70 \%$ ethanol and all items were cleaned with $70 \%$ ethanol prior to placing them into the hood. All the materials that were used were placed under the laminar flow hood (except media, solutions and micropipettes) and exposed to UV light for 15 minutes to sterilize the surfaces. Media, pipettes and micro-pipettes are sensitive to UV light and may denature if exposed, therefore they were not exposed to the light.

\subsection{Preparation of Reagents}

\subsection{1 aMEM for Cell Culture}

Ascorbic acid free MEM Alpha Media (Cat. No. A1049001, Gibco® by Invitrogen Carlsbad, California) was used for culturing and passaging pre-osteoblast cells. The media was prepared for use with cell cultures by adding previously aliquoted container of FBS (50ml tube) and antibiotics $(5 \mathrm{ml})$ to new sterile $500 \mathrm{ml}$ bottles of $\alpha \mathrm{MEM}$. The prepared media was then well mixed.

\subsubsection{Ascorbic Acid (50 $\mathrm{\mu g} / \mathrm{ml})$ - Cell Differentiation Supplement}

The desired concentration of AA in cell differentiation media was $50 \mu \mathrm{g} / \mathrm{ml}$. A stock solution of $50 \mathrm{mg} / \mathrm{ml}$ was prepared. Five hundred $\mathrm{mg}$ of L-ascorbic acid was weighed out (Cat. No. A5960-25G, Sigma Aldrich Inc., Oakville, Ontario) and place into a sterile flask. Ten ml of 
sterile PBS ( $\mathrm{CaCl}_{2}$ and $\mathrm{MgCl}_{2}$ free) was filtered, using a $0.22 \mu \mathrm{m}$ filter, into the flask. The PBS and AA were mix thoroughly. When the AA had completely dissolved, the solution was then filtered through a new sterile $0.22 \mu \mathrm{m}$ filter into a new sterile flask. Since AA is light sensitive, the flask was immediately wrapped in foil to prevent light penetration. The stock solution was then $1000 \mathrm{x}$ concentration; therefore for every $1 \mathrm{ml}$ of media added to a cell culture, $1 \mu \mathrm{l}$ of stock solution was added.

\subsection{3 $\quad \beta$-Glycerol Phosphate (3mM) - Cell Differentiation Supplement}

The desired concentration for $\beta$-Glycerol Phosphate ( $\beta$-GP) in differentiation media was $3 \mathrm{mM}$. The molecular weight of $\beta$-GP is 216.04 . Therefore $1 \mathrm{M}$ of $\beta$-GP is $216.04 \mathrm{~g} / \mathrm{l}$ or 216.04 $\mathrm{mg} / \mathrm{ml}$. A 3mM concentration of $\beta$-GP would then be $0.648 \mathrm{mg} / \mathrm{ml}$. The stock solution was prepared by weighing out $648 \mathrm{mg}$ of Glycerol 2-phosphate disodium salt hydrate (Cat. No. G9891-10G, Sigma Aldrich Inc., Oakville, Ontario) into a sterile flask. Ten ml of sterile PBS, $\left(\mathrm{CaCl}_{2}\right.$ and $\mathrm{MgCl}_{2}$ free) was then filtered into the sterile flask using a $0.22 \mu \mathrm{m}$ filter. The PBS and $\beta$-GP were then thoroughly mixed. When the $\beta$-GP had completely dissolved, the solution was then filtered through a new sterile $0.22 \mu \mathrm{m}$ into a new sterile flask. The stock solution was $100 \mathrm{x}$ concentration, therefore for every $1 \mathrm{ml}$ of media added to a cell culture, $10 \mu \mathrm{l}$ of stock solution was added.

\subsubsection{Differentiation Supplement for aMEM Media}

For cell cultures to differentiate from pre-osteoblasts to mature osteoblasts AA and $\beta$-GP must be present in the cell culture media. The $\alpha$-MEM use to induce differentiation in cell cultures is called Differentiation Supplement (DS) positive media. To ensure the DS ingredients were active in the media, the solution was mixed less than 1 hour prior to use. DS+ $\alpha$-MEM 
media was made by adding $1 \mu \mathrm{l}$ of AA stock solution and $10 \mu \mathrm{l}$ of $\beta$-GP stock solution for each $\mathrm{ml}$ of $\alpha$-MEM. The DS+ media had a final concentration of $50 \mu \mathrm{g} / \mathrm{ml}$ of AA and 3mM of $\beta-\mathrm{GP}$. The solution was mixed thoroughly before use.

\subsubsection{Alizarin Red for Cell Culture Staining}

The desired concentration for alizarin red staining was $1 \mathrm{mg} / \mathrm{mL}$. A stock solution of 10 mg/mL was made. The stock solution was prepared by weighing out $500 \mathrm{mg}$ of Alizarin Red-S (Cat. No. A5533-25G, Sigma Aldrich Inc., Oakville, Ontario) into a sterile flask. Fifty ml of degassed filtered water was added to the flask. The alizarin red and water were mixed thoroughly until the alizarin red had dissolved. The stock solution was $10 \mathrm{x}$ concentration; therefore to dilute the solution, $1 \mathrm{ml}$ of stock solution was added to every $10 \mathrm{ml}$ degassed filtered water. The bonding of alizarin red to calcium is $\mathrm{pH}$ dependant, therefore it is critical that the $\mathrm{pH}$ is adjusted a pH of 4.2 prior to use (Puchtler et al 1969, Davey and Lord 2003). The pH was adjusted with sodium hydroxide $(\mathrm{NaOH})$ and hydrochloric acid $(\mathrm{HCl})$. Once the $\mathrm{pH}$ was adjusted, the stock solution had a shelf life of 1 year.

\subsubsection{Perchloric Acid for Alizarin Red Stain Rehydration}

The desired concentration for perchloric acid was 5\%. A stock solution of Perchloric Acid ACS reagent 60\% (Cat. No. 311413, Sigma Aldrich Inc., Oakville, Ontario) was purchased. The concentration degassed filtered water. 


\subsection{Cell Maintenance}

\subsubsection{Splitting and Passaging Cells}

The cells were grown in a cell culture flask until they were confluent, usually 2-4 days. Once the cells were confluent they could be re-suspended for passaging or seeding.

\subsubsection{Digesting and Re-Suspending Cells}

First the old media was aspirated from the culture flask using a sterile suction pipette. Then enough PBS ( $\mathrm{CaCl}_{2}$ and $\mathrm{MgCl}_{2}$ free) was added to cover the cell culture to a depth of about $0.5 \mathrm{~mm}$ deep. The cells were then washed for 30 seconds by gently rocking the PBS over the

culture (about $0.1 \mathrm{ml}$ for every $\mathrm{cm}^{2}$ ). This removed any excess media and debris that might have been left on the culture plate. Any remaining media may prevent the cells from being digested. The PBS ( $\mathrm{CaCl}_{2}$ and $\mathrm{MgCl}_{2}$ free) was aspirated from the culture using a sterile suction pipette. Just enough trypsin was added to the flask to cover the cell culture. The flask was then rocked gently until the entire culture was covered with trypsin. The cell culture was then incubated at $37^{\circ} \mathrm{C}$ for $5-10$ minutes to speed up the digestion process. About half way through the digestion process cell culture was checked to ensure that the entire cell surface was covered with trypsin. After 10 minutes of incubation the culture was tested for cell detachment. The testing was done by tilting the plate slightly and then placing it back on the microscope stage. If the cells continue to move once the culture plate had been replaced on the microscope stage, then the cells were confirmed to have detached. If the cells were not detached, the flask was replaced in the incubator for an additional 5 minutes. This process was repeated every 5 minutes until the cells detached. 
Once the cells had detached from the culture plate, enough warmed $\left(37^{\circ} \mathrm{C}\right)$ media was added to cover the cell culture surface (about $1 \mathrm{ml}$ of media for each $1 / 2 \mathrm{ml}$ of trypsin). The addition of the media neutralized the trypsin and stopped the digestion process. A sterile transfer pipette was used to mix trypsin and media. This mixture was then washed over the cell culture surface several times until all cells had detached from the plate.

A transfer pipette was used to move the mixture of digested cells, trypsin and media to a sterile centrifuge tube. Enough media was added to the centrifuge to so that the total volume was $10 \mathrm{ml}$. To separate the cells from the supernatant, the mixture was centrifuged at 900 RPM for 8 minutes. This made cells form a clump at the bottom of the tube called a cell pellet. When the centrifuge tube was removed from the centrifuge, the sample was checked for cell pellet formation at the bottom of the tube. The centrifuge separated the cells from the supernatant.

The supernatant was carefully aspirated so that only the cell pellet remained. When only the pellet remained, a small amount of media was added to the pellet. A sterile transfer pipette was used to mix the pellet and re-suspend the cells. This total volume of suspended cells was used to assess the total number of cells.

\subsubsection{Counting Cells}

The cell pellet was thoroughly mixed into the media to ensure accurate cell counts. Using a micro-pipette, $10 \mu \mathrm{l}$ of the sample volume mixture was injected under the slip cover of the hemocytometer plate. The hemocytometer was then placed under the microscope so the square labeled A in Figure 3-1 could be seen (each square is $1 \mathrm{~mm} \times 1 \mathrm{~mm}$ ). All the cells in square A were counted. The process was then repeated for squares labeled B, C, and D in Figure 3-1. A sum of all four squares was then calculated. This sum and the sample volume were used 
in equation 3.1 to calculate the total number of cells. A portion of these was used both to passage cells and to seed them for experiments. To calculate the volume of cells needed for seeding equation 3.2 was used.

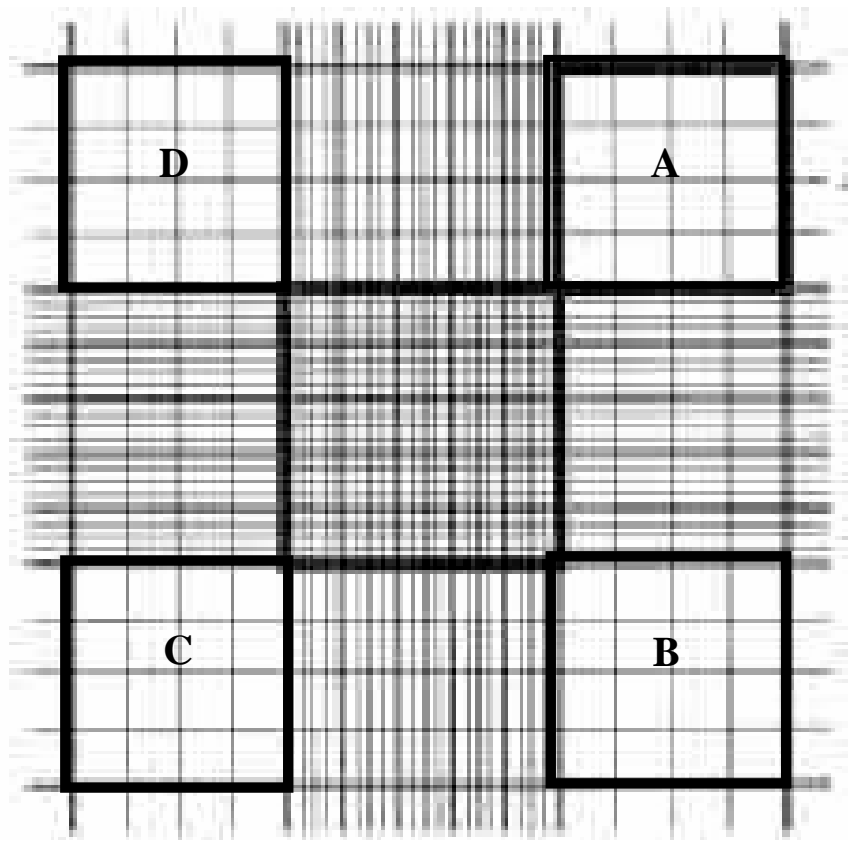

Figure 3-1: Hemocytometer grid (Maes 2004).

To calculate the total number of cells

Total cells $=\frac{\text { Total cells counted }}{4} \times 10^{4} *$ total volume of sample

To calculate the desired volume of cells from the sample

desired volume $=\frac{\text { Total cells }}{\text { sample volume }} \times$ desired cells

The cells were seeded at $\approx 0.5 \times 10^{5} \mathrm{cells} / \mathrm{ml}$ to passage for proliferation. For experiments the cell seeding procedure is described below. 


\subsubsection{Seeding Cells - 6 Well Plate}

For these experiment cells were seeded into 6 well plates (Cat. No.Z707767, Sigma Aldrich Inc., Oakville, Ontario). Each well was $35 \mathrm{~mm}$ in diameter. A total of $2 \mathrm{ml}$ of $\alpha$-MEM media was added to each well. The media added to each well was either DS+ or DS-. The DS+ media was used to differentiate cells from pre-osteoblasts to osteoblasts. The cells continued to receive the same type of media throughout the treatment as they received during seeding.

The total amount $\alpha$-MEM needed was aliquoted into a sterile flask and warmed at $37^{\circ} \mathrm{C}$ for 15 minutes. Once the solution had warmed, $1.5 \mathrm{ml}$ of media was added to each well. The total desired volume of cells was then added to the remaining media. The cells and media were then mixed with a transfer pipette. Then $0.5 \mathrm{ml}$ of the cell-media mixture was added to each well. After seeding 6 wells, the cell suspension was remixed. This helped keep the seeding even. After all the wells had been seeded with cells, each culture plate was gently rocked about 10 times. This helped to distribute the cells evenly throughout the well. After seeding all the wells, each well was check under the microscope to ensure that there were cells in each well.

Once the cells had been seeded the media was changed every third or fourth day. On a 7 day cycle this would mean that the media will be changed every Monday and Thursday.

\subsection{Fixing cell culture and Staining for mineralization}

To fix the cell cultures, the media was aspirated from the wells using a sterile suction pipette. The cultures were then rinsed with $1 \mathrm{ml} /$ well $\mathrm{PBS}\left(\mathrm{CaCl}_{2}\right.$ and $\mathrm{MgCl}_{2}$ free). The $\mathrm{CaCl}_{2}$ and $\mathrm{MgCl}_{2}$ free PBS was use so that no extra $\mathrm{Ca}^{+2}$ was added to the culture. The PBS was aspirated and then the cultures were rinsed 2 more times. After rinsing the wells $1 \mathrm{ml} /$ well of $10 \%$ formalin at room temperature $\left(20^{\circ} \mathrm{C}\right)$ (Cat. No. F5304, Sigma Aldrich Inc., Oakville, 
Ontario) was added to each well. The cell culture plates were gently rocked for approximately $10 \mathrm{~s}$ to ensure the culture is evenly covered with formalin. The formalin incubated at room temperature for 20 minutes. The excess formalin was aspirated and the cultures were rinsed 3 more times with $1 \mathrm{ml} /$ well $\mathrm{PBS}\left(\mathrm{CaCl}_{2}\right.$ and $\mathrm{MgCl}_{2}$ free). At this point the cells were fixed.

Then $1 \mathrm{ml}$ of $1 \mathrm{mg} / \mathrm{ml}$ alizarin red at room temperature $\left(20^{\circ} \mathrm{C}\right)$ was added to each well to stain the cell cultures. The culture plates were then gently rocked for approximately $10 \mathrm{~s}$ to ensure the cultures were evenly covered with alizarin red. The alizarin red incubated on the cell cultures for 20 minutes at room temperature. After incubation the excess alizarin red was aspirated from the wells and the cultures were rinsed 3 more times with $1 \mathrm{ml} / \mathrm{well} \mathrm{PBS}\left(\mathrm{CaCl}_{2}\right.$ and $\mathrm{MgCl}_{2}$ free). Washing the cultures with $\mathrm{PBS}\left(\mathrm{CaCl}_{2}\right.$ and $\mathrm{MgCl}_{2}$ free) removed the nonspecific stain association with calcium (Stanford et al 1995). The cultures were left to dry for 24 hours. The cultures were then ready to photograph.

\subsection{De-Staining and Quantification}

When the stained cultures had dried for 24 hours, they were ready for de-staining (once the stain had dried it could be left for several days prior to de-staining). To de-stain the culture 1 $\mathrm{ml}$ of room temperature $5 \%$ perchloric acid was added to each well. The perchloric acid rehydrated and dissolved the stain culture for 23 hours (the de-staining time remained consistent over all experiments). After de-staining for 23 hours, the perchloric acid was removed from the wells.

Using a micropipette $5 \times 100 \mu \mathrm{l}$ samples were taken from each well. Then each $100 \mu \mathrm{l}$ sample was placed into a single well on a 96 well plate (see Figure 3-2 for details). To quantify

the degree of staining, the 96 well plate was put through a plate reader. A Thermo Lab Systems 
Multiskan Ascent and Ascent software (Cat. No. 51118300 and 51118307, Thermo Fischer, Franklin, MA.) plate reader was used to measure absorbance. Absorbance for each well was read at $405 \mathrm{~nm}$. The average of 5 wells was considered the absorbance for that sample.

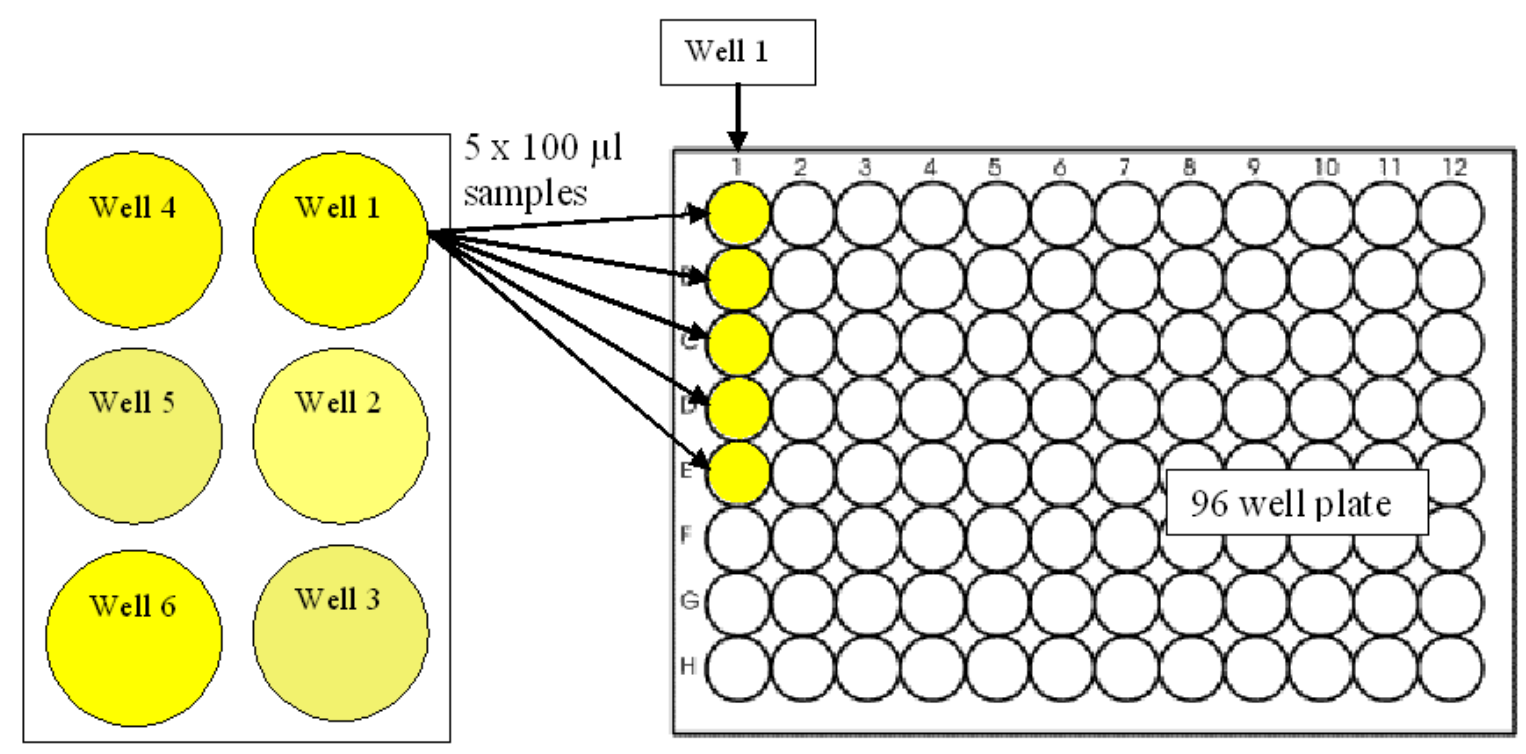

Figure 3-2 Sample selection for spectrometer measurements. Remove the remove the solution from the 6 well plate after 23 hours incubation at room temperature.

\subsection{Cell Treatment Experimental Protocol}

The experimental protocol was developed in collaboration with the R\&D department of Smith \& Nephew Inc. In all experiments cells were seeded 72 hours prior to treatment. This allowed the cells time to proliferate, adhere to the well plate surface. DS+ or DS- media was added to the wells in the experiments. The cell cultures receive treatment for up to 15 days.

The experiments consisted of 4 treatment groups: control, LIPUS, LIPUS + heat, and heat. All treatment groups were grown on polystyrene 6 well plates with a well diameter of 3.5 $\mathrm{cm}$. All cells were treated in a 7 day cycle with 5 days of treatment and 2 days off. Samples were taken out of treatment groups on day 5 of the cycle unless otherwise noted. Outside of 
treatment all groups were kept at $37^{\circ} \mathrm{C}$ with $5 \% \mathrm{CO}_{2}$. All the cell plates were cleaned with $70 \%$ ethanol after treatment or handling and before being replaced into the holding incubator.

The schematic in Figure 3-3 illustrates the experimental set up. The transducer was placed $13 \mathrm{~mm}$ below the cell culture well and coupled to the cell culture well using $37^{\circ} \mathrm{C}$ water. The cell plate was held in place with a fixture. The cells were held in place above transducer, so that the bottom of the cell plate was always in contact with the water. The water tank was kept inside the test incubator (a separate incubator was used to apply treatment so the untreated cell cultures would not be affected).

The LIPUS device used to apply treatment was a system of Exogen ${ }^{\circledR}$ treatment transducer used specifically for research (described in detail in chapter 2). An $\mathrm{I}_{\mathrm{SATA}}=10$ $\mathrm{mW} / \mathrm{cm}^{2}$ (see chapter 2, section 2.2.8, 2.3.4, 2.4 for details of intensity reduction) was delivered to each sample well by a single flat unfocused transducer. The effective radiating area of the transducer was $4.71 \mathrm{~cm}^{2}$ (seed chapter 2 for details on effective radiating area). The LIPUS cell cultures were treated with a frequency of $1.5 \mathrm{MHz}$, a PRF of $1 \mathrm{kHz}$ and a $200 \mu$ s pulse. All wells on the 6 well plate were treated simultaneously and driven by the same power source. For the combine LIPUS + heat treatment, the incubator and water temperature were increased to 40.5 $\pm 0.5^{\circ} \mathrm{C}$ prior to treatment; otherwise the set up was left the same as for the treatment receiving only LIPUS. For heat treatment alone the LIPUS device was disconnected from the power source and the incubator and water temperature were increased to $40.5 \pm 0.5^{\circ} \mathrm{C}$ prior to treatment. The control cell culture group remained in the holding incubator. 


\section{Incubator}

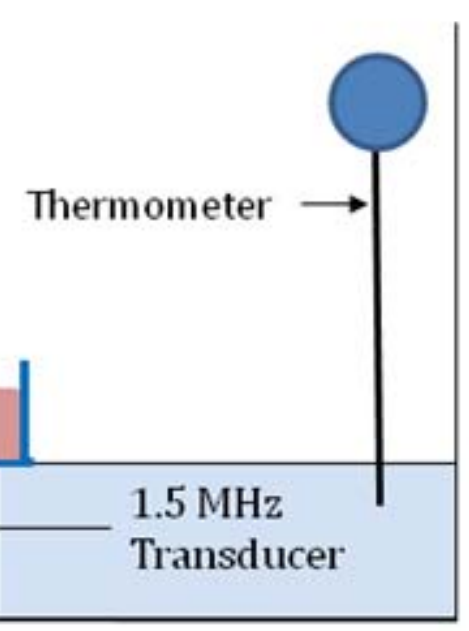

Water Tank

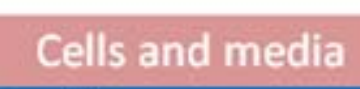

$13 \mathrm{~mm} \downarrow$

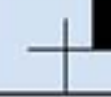

\section{Experimental set up}

Figure 3-3 Schematic of cell treatment set up

\subsubsection{Cell Culture - Pre Experiment Testing}

\subsubsection{Differentiation}

The process of cell differentiation from pre-osteoblasts to mature osteoblasts in cell cultures includes several distinct phases. The progress of differentiation was followed through a series of observations and tests. Earlier phases of differentiation were assessed through visual inspection under the microscope or with the naked eye and, cell counts were made. Later stages of differentiation were assessed visually and with alizarin red cell culture staining.

All bone cells need the addition of AA to differentiate from pre-osteoblast cells to osteoblast cells. The onset of mineralization is associated with the end stages of differentiation. 
To enhance mineralization and decrease time to differentiation $\beta$-GP was added to the cell culture media.

\subsubsection{Initial Signs of Differentiation}

The initial stages of differentiation follow a distinct physiological pattern. The early signs of differentiation include change in morphological features, cell proliferation and collagen matrix deposition. The changes in morphological features were observed by imaging cells using a Q-Imaging Retiga 2000R camera attached to an Olympus CKX41 inverted microscope (Olympus, Markham, Ontario). Pictures of the cell cultures were taken under at 40X magnification and compared to previously published values.

A quantitative analysis of proliferation was made by counting the cells on day 1, 3, 5 and 12. The cells were counted using Trypan Blue (Cat. No. T6416, Sigma Aldrich Inc., Oakville, Ontario). The cells were removed from suspension by digesting the cells (as described in section 3.5.2). After suspending the cells, a $50 \mu \mathrm{l}$ sample was taken and $10 \mu \mathrm{l}$ of trypan blue was added to the suspended cells. The cells were counted according to the method described in section 3.5.3. A total of 2 samples were taken on each day and the averages of the samples were used.

Collagen matrix deposition was inferred in two ways. First, when the media was aspirated from the well, the collagen matrix would lift off the cell plate intact. Pictures of where the collagen sheet lifted off the well plate were taken with a digital camera. Second, the collagen matrix would not digest in trypsin. The entire collagen sheet was removed from the plate and a picture was taken. 


\subsubsection{Later Signs of Differentiation - Calcification and Improved Mineralization}

As noted above, the final stages of differentiation are associated with the onset of mineralization. The optimum differentiation and ultimately mineralization were attained by adjusting the concentrations of AA and $\beta-G P$. The optimum concentrations for cell cultures in this experiment were obtained by varying the amount of supplement from $0-100 \mu \mathrm{g} / \mathrm{ml}$ for AA and 3-5 mM for $\beta$-GP. The cells were left in culture for 15 days and the media was changed every 3-4 days. The DS concentration of the culture that showed the greatest mineralization on day 15 was used to set levels for all the cultures in future experiments.

Differentiation and the onset of mineralization were assessed using alizarin red staining. Qualitatively cell cultures were considered to be positively stained for mineralization when they stain red. Quantitatively, the cells de-stained and assessed according to the method described in section 3.7. The cultures with the highest averaged absorbance were considered the most mineralized.

\subsubsection{Confirmation of Differentiation}

To confirm the DS+ media was effective in maturing and mineralizing the cell cultures, DS+ media cell cultures were tested against a control with DS- media cell cultures. The cell cultures were considered more mature and differentiated if the DS+ cell cultures were statistically significantly more mineralized than their DS- cell culture controls. As described in sections 3.4.5 and 3.4.6, mineralization was measured through the absorbance of alizarin red staining. 
There were four treatment groups included in this trial. Control (C), LIPUS for 20 minutes (US20), LIPUS for 20 minutes then a 1 hour break then Heat at $40^{\circ} \mathrm{C}$ for 60 minutes (US20 + H60), and Heat for 60 minutes (H60). The samples were taken on days 5, 10 and 15 .

In addition to testing for differentiation, the DS+ media cell cultures were assessed for treatment effect, by comparing the US20, US20 + H60, and H60 to the Control cell culture. The treatment was considered to have an effect if the culture was statistically significantly more mineralized that Control.

\subsubsection{LIPUS Treatment Effect - Comparison to Published Results}

The effect of LIPUS on bone cells cultures has been previously published using an LIPUS device identical to the one used in this experiment (Unsworth et al 2007). In this experiment the assessment of the LIPUS treatment was made by comparing the effect of a 20 minute LIPUS dose (US20) to an untreated Control (C). The sample included 12 DS+ cell cultures and 6 DS- cell cultures in each treatment group. The samples were taken on days 5, 10 and 15 .

First, both treatment groups needed to show the DS+ cell cultures were statistically significantly more mineralized than their same treatment DS- counterpart. If this was true, then the cultures could be considered differentiated. If both groups were found to be differentiated, then the US20 treatment was considered to be effective if it was statistically more significantly mineralized that the Control treatment.

\subsubsection{Test of Hypothesis - Combined Treatment effect}

Although the individual effects of ultrasound and heating are known to increase mineralization in bone cell cultures, the combined effect of heating and ultrasound on bone cells 
has not been examined previously. The treatments were as follows Control (C) received no treatment, LIPUS for 40 minutes (US40), LIPUS for 40 minutes and Heat at $40^{\circ} \mathrm{C}$ for 40 minutes concurrently (US40 $+\mathrm{H} 40$ ) and Heat at $40^{\circ} \mathrm{C}$ for 40 minutes (H40). The experiment was repeated 3 times with 6 replicates in each sample. Each run of the experiment was seeded with a different passage of cells. The samples were taken on days 0, 5, 10 and 15.

For this experiment, differentiation was assessed by comparing the mineralization of day 5 samples to the mineralization of day 0 samples. The day 5 samples were considered to be differentiated if they were statistically significantly more mineralized that the day 0 sample. If all groups were found to be differentiated, then the treatments were considered to be effective if they was statistically more significantly mineralized that the Control treatment.

\subsection{Spectral Measurements of Alizarin Red}

A mineralized matrix is primarily calcium therefore alizarin red was used to assess the degree of mineralization in a cell culture. Mineralization was measured through the absorption of alizarin red. Once a cell culture is fixed, stained and dried this indirect measurement could be made. The dye alizarin red binds preferentially to calcium, 1 mole of alizarin red selectively binds to 2 moles of calcium (Stanford et al 1995, Yang et al 2005). The molar absorptivity for alizarin red is $10^{-4} /(\mathrm{mol} \times \mathrm{cm})=2.1($ valid for $0.05-5.5 \mu \mathrm{g})($ Stanford et al 1995). The peak absorbance for alizarin red is $420 \mathrm{~nm}$ (Csányi 1980).

Once the 96 well plate was prepared (Figure 3-2), the entire plate was inserted into the plate reader. The plate reader produced a single wave length of light at $405 \mathrm{~nm}$. Anything that was highly absorbent at $405 \mathrm{~nm}$ would change absorbance of the sample. To ensure that the dissolved alizarin red was the only significant absorber was at $405 \mathrm{~nm}$ and to ensure that $405 \mathrm{~nm}$ 
was close enough to the spectral peak of alizarin red; multiple full spectrum measurements were made.

Several cultures containing alizarin red were run through and Perkin Elmer UV VIS spectrometer Lambda 20 -2 nm (Perkin Elmer, Norwalk CT) with UV WIN Ware with light wave lengths from 300-800 nm. The experimentally measured peaks were compared to literature values of peak absorbance of alizarin red. In addition, samples containing different dummy solutions (waster, perchloric acid, and alizarin red or waster, perchloric acid, alizarin red and formalin) were compared to solutions taken from cell culture samples to ensure there were no other dominant absorbers at $405 \mathrm{~nm}$.

3.10 Statistical Study Design (Blair and Taylor 2008)

\subsubsection{Comparison of LIPUS and Heat to Control}

Earlier work has shown that the addition of LIPUS or the addition of heat to bone cells would increase mineralization. To establish this trend the null hypothesis is that the addition of LIPUS treatment to cell cultures will not increase mineralization when compared to a control cell cultures and therefore the means will be equal. The assumptions include that individual cultures are independent, and the population is normally distributed.

Let $\quad \mathrm{H}_{0}: \mu_{0}=\left(\mu_{\mathrm{C}}-\mu_{\mathrm{US}}\right)=0$

$\mathrm{H}_{\mathrm{a}}: \mu_{\mathrm{a}}=\left(\mu_{\mathrm{C}}-\mu_{\mathrm{US}}\right) \geq 0.005$

$\alpha=0.05$

and

Let $\quad \mathrm{H}_{0}: \mu_{0}=\left(\mu_{\mathrm{C}}-\mu_{\mathrm{H}}\right)=0$ 


$$
\begin{aligned}
& \mathrm{H}_{\mathrm{a}}: \mu_{\mathrm{a}}=\left(\mu_{\mathrm{C}}-\mu_{\mathrm{H}}\right) \geq 0.005 \\
& \alpha=0.05
\end{aligned}
$$

Where $\mathrm{H}$ indicates the hypothesis, $\mu$ indicates the treatment mean, $\alpha$ is the probability that the statistical tests will generate a false positive error (rejecting the null hypothesis even though it is true). The subscripts are indicated as follows: US = LIPUS, $\mathrm{H}=$ heat, $\mathrm{C}=$ control.

The null hypothesis of equal means will be tested using the Two Independent Samples Method (Student's t test).

$$
t=\frac{\bar{x}_{c}-\bar{x}_{u s}-\delta_{0}}{\sqrt{s_{P}^{2}\left(\frac{1}{n_{1}}+\frac{1}{n_{2}}\right)}}
$$

Where $t$ is the test statistic, $x$ represents the mean, $\delta$ is the difference between means, $s_{p}{ }^{2}$ is the pooled variance and $n$ is the treatment population. The degrees of freedom are determined (dof) are defined as:

$d o f=n_{1}-n_{2}-2$

The variance is calculated as follows:

$s_{P}^{2}=\frac{\left(n_{1}-1\right) s_{1}^{2}+\left(n_{2}-1\right) s_{2}^{2}}{n_{1}-n_{2}-2}$

Where the individual variance are calculated with:

$S=\sqrt{\frac{\sum x^{2}-\left(\sum x\right)^{2}}{n-1}}$

For completeness, LIPUS + heat will also be compared to the control in the same manner as mentioned above. 


\subsubsection{Comparison of LIPUS + Heat to LIPUS}

The null hypothesis of this experiment is that the addition of heat to LIPUS will not increase mineralization of cell cultures and therefore the treatment mean between LIPUS treatment and LIPUS + heat treatment will be equal. A student t-test will be used to test for equal means. The assumptions include that individual cultures are independent, and the population is normally distributed.

Let $\quad H_{0}: \mu_{0}=\left(\mu_{U S}-\mu_{U S+H}\right)=0$

$\mathrm{H}_{\mathrm{a}}: \mu_{\mathrm{a}}=\left(\mu_{\mathrm{US}}-\mu_{\mathrm{US}+\mathrm{H}}\right) \geq 0.005$

$\alpha=0.05$

The subscript US+H indicates a treatment of LIPUS and heating concurrently.

The method for calculating the statistics will be the same as mention in section 3.10.1, using equations from 3.3-3.6. 


\section{Chapter 4 Cell Culture Experiments: Results}

\subsection{Differentiation}

The process of differentiation is the maturation of cells in culture from pre-osteoblasts to osteoblasts. For pre-osteoblast cells to differentiate into an osteoblast cells, they must go through 3 distinct physiological stages. First the cells proliferate, then a collagen matrix is laid down and finally the collagen matrix mineralizes and calcium nodules begin to form (Beck Jr. et al 2000). During the proliferations stage of differentiation, the cells change from a flat monolayer of fibroblastic looking cells to densely packed round cells. The cells will continue to proliferate until they are confluent. As proliferations ends, a collagen matrix forms. During this phase, collagen molecules begin to form bonds holding the cells in a flexible matrix. By this stage of differentiation, dislodging the cells from the collagen matrix becomes difficult. As cell differentiation reaches its final stages and cells mature, minerals, primarily calcium, begin to deposit in the collagen matrix. Mineralization is the last physiological stage in the differentiation process, however mineralization will continue even after the cells have fully matured.

\subsubsection{Initial Signs of Differentiation}

Sudo et al (Sudo et al 1983) demonstrates (Figure 4-1 A and B) that primary MC3T3 E1 cells between day 2 and day 6 cells have changed from fibroblastic looking cells to a proliferated mosaic of cells with clearly defined nuclei. When this same type of cell line was grown for these experiments, the immortalized MC3T3 - E1 cells showed similar morphological expressions (Figure 4-1 C and D). Initially, $10^{5}$ cells were plated in each well, for a density of 
$0.5 \times 10^{5}$ cells $/ \mathrm{ml}$. The cell population grows by almost 15 times over the first 2 days, by almost 2 times over the next 2 days and by less than 1 time over the next two days. Figure 4-2 shows that the rate of cell proliferation significantly declines around day 6 .
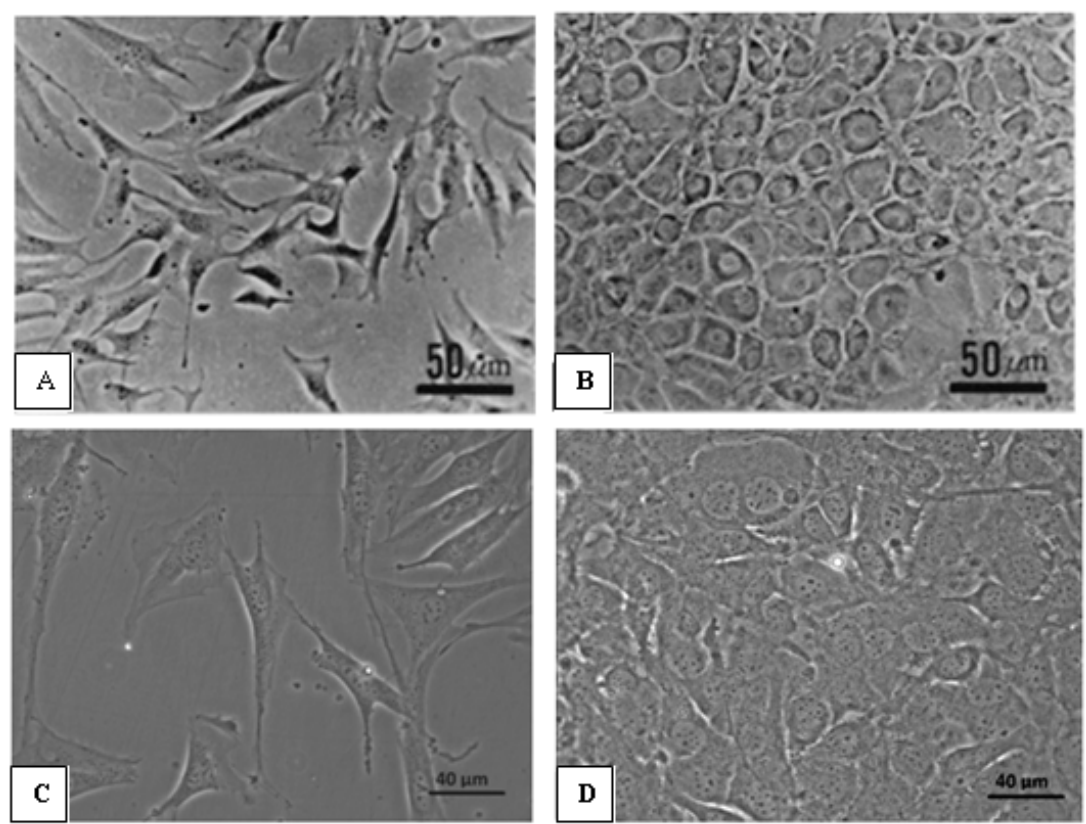

Figure 4-1: Primary MC3T3-E1 cells A) 2 days after seeding B) 6 days after seeding. Images from (Sudo et al 1983). Immortalized MC3T3-E1 from ATCC C) 2 days after seeding D) 6 days after seeding.

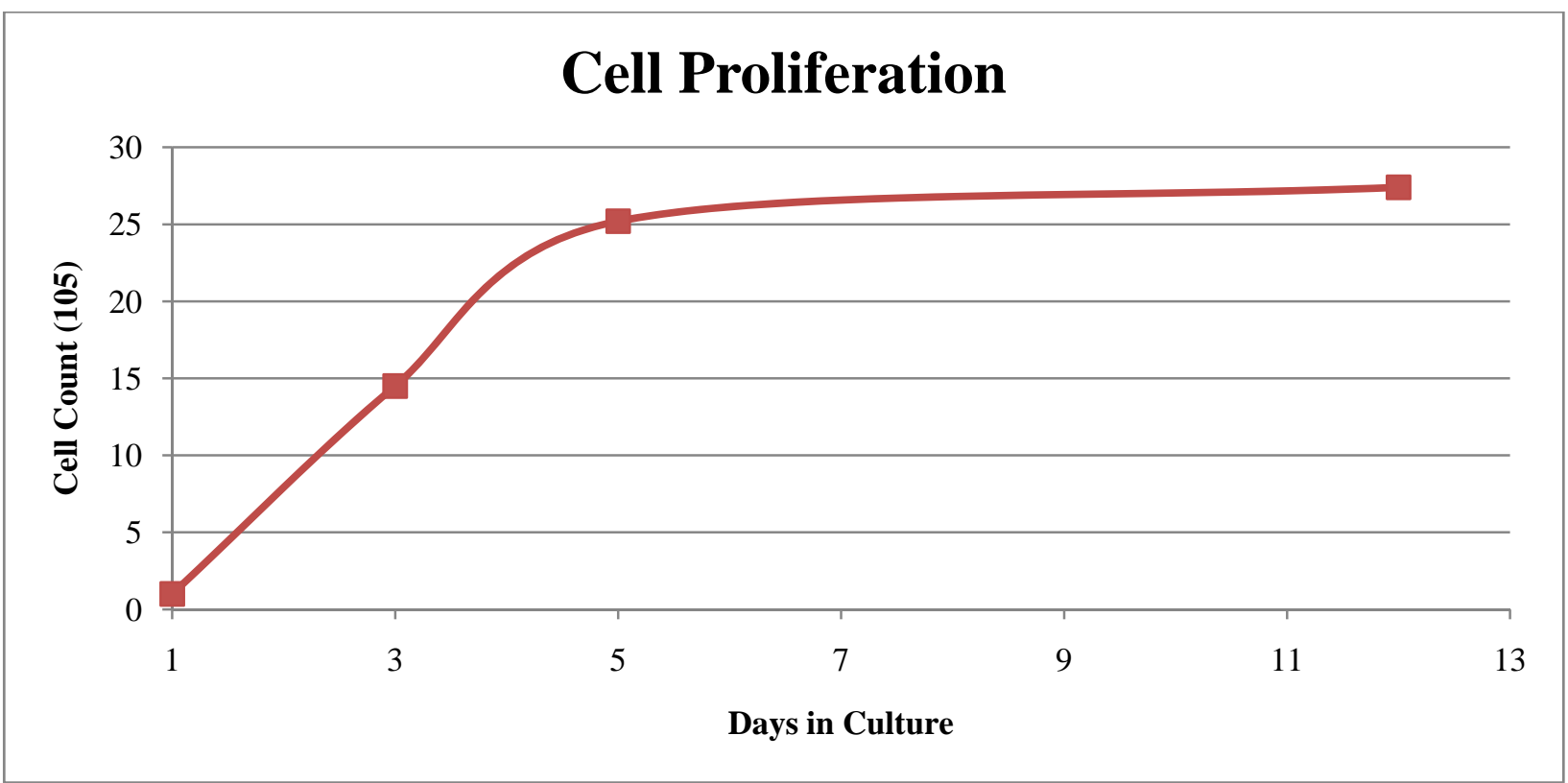

Figure 4-2: Cell Proliferation rates. 
A definitive indication of collagen matrix formation is usually done with staining. This testing was not done in this thesis, however there were observed indications of the cell culture changing and a collagen matrix forming. When the media was aspirated from the differentiated cell culture wells, the sheet of collagen would lift off at the edge of the culture dish (Figure 4-3 A). On the other hand, the undifferentiated cultures would not show the same feature (Figure 4-3 B). In addition, digestive enzymes like trypsin could not break down the collagen matrix (Figure 4-3 C). Trypsin was easily able to break apart the cells in a DS- culture. The culture stayed intact as it was removed from the well plate and had a mucous type appearance. The observed appearance of these features suggests that a collagen matrix was forming.
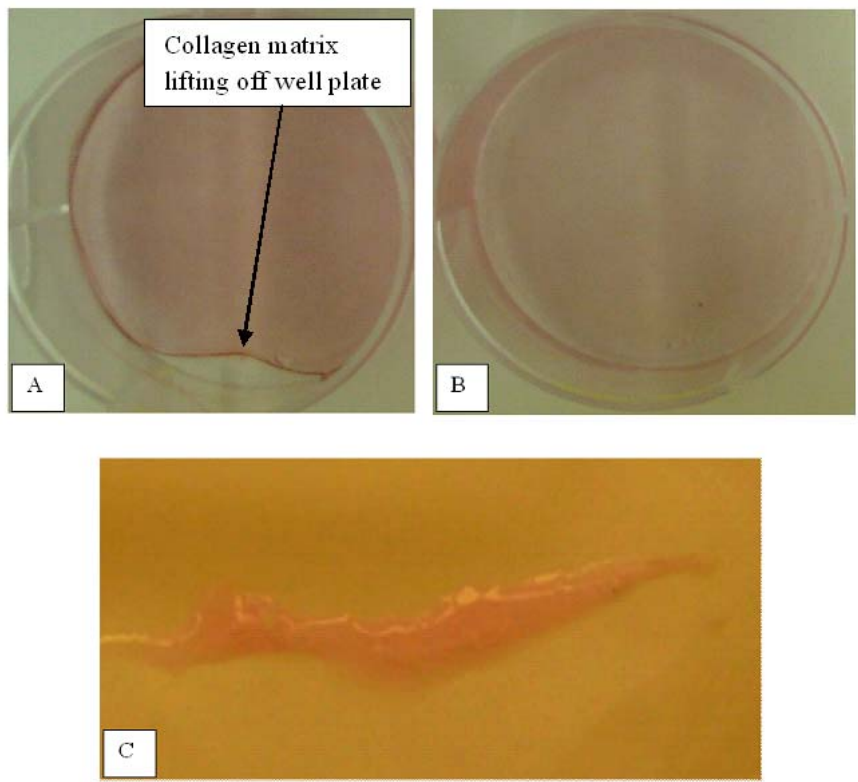

Figure 4-3: Day 15, MC3T3-E1 cell cultures A) Cell culture with differentiation supplement added. B) Cell culture without cell differentiation supplement $C$ ) Intact collagen matrix of differentiation supplement positive cell culture.

As the bone cells differentiate and mature, minerals begin to accumulate within the collagen matrix. As seen in Figure 1-10, imaging early stages of mineralization need a high degree of magnification or the cultures must remain approximately 40-50 days to be able to see calcium nodules form with the naked eye (Sudo et al 1983). 


\subsubsection{Later signs of Differentiation - Calcification and Improved Mineralization}

Whether maturing from mesenchymal stem cells or pre-osteoblasts, all bone cells need Ascorbic Acid (AA) to differentiate and become mature osteoblasts. AA is essential for a collagen matrix to form and the collagen matrix is need for mineralization to form in bone or bone cell cultures (Wang et al 1999). Cultures supplemented only with AA may require a up to 50 days to mineralize sufficiently to become measurable (Sudo et al 1983). The addition of $\beta$ Glycerol Phosphate ( $\beta$-GP) will enhance the rate mineralization and therefore reduce the length of time a culture needs to begin mineralization (Franceschi et al 1994).

To improve the degree of mineralization in the cell culture, varied combinations of concentration of AA and $\beta$-GP are added to the cell culture. The concentration of AA was varied from 50-100 $\mu \mathrm{g} / \mathrm{ml}$ and $\beta$-GP was varied from 3-5 $\mathrm{nM}$ (Wang et al 1999). If the cultures were strongly mineralized the staining could be detected with the naked eye (Figure 4-4) or through a microscope (Figure 4-5). The photograph of the alizarin red stained wells in Figure 4-4 correlates with the magnified images in Figure 4-5, where the magnified dark red patches indicated mineral deposits. Spectrometer measurements (Figure 4-6) were able to detect more subtle changes in the alizarin red staining and therefore the mineral content. Both the visual and spectrometer inspections suggested that $50 \mu \mathrm{g} / \mathrm{ml}$ of AA with 3nM of $\beta$-GP and $50 \mu \mathrm{g} / \mathrm{ml}$ of AA with $4 \mathrm{nM}$ of $\beta$-GP were both found to mineralize by day 15 (Figure 4-4 and Figure 4-6). Spectrometer measurement at $405 \mathrm{~nm}$ revealed that there was slightly higher mineralization for $50 \mu \mathrm{g} / \mathrm{ml}$ of AA with $3 \mathrm{nM}$ of $\beta-\mathrm{GP}$. 


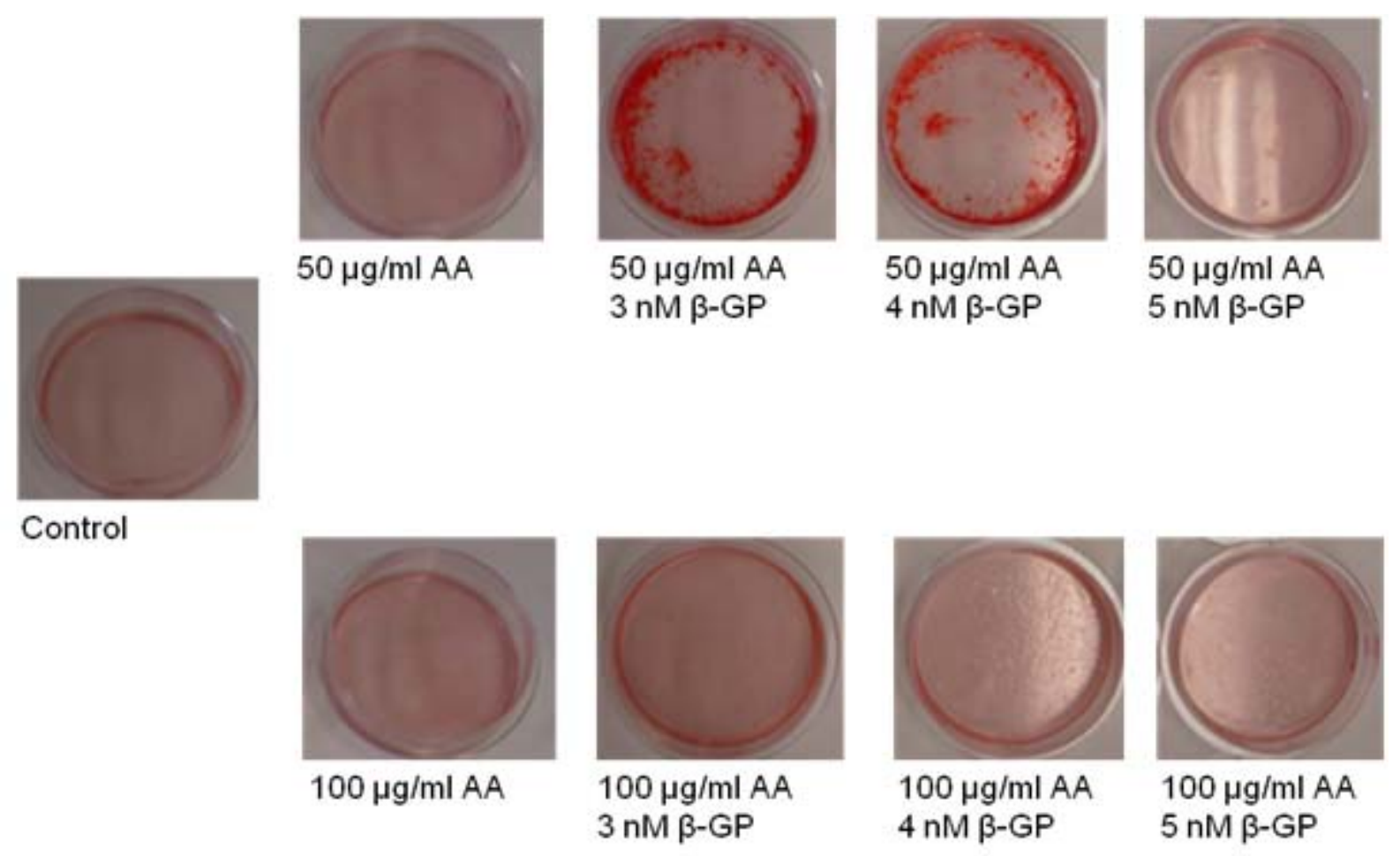

Figure 4-4: MC3T3-E1 alizarin red stained cell cultures on Day 15. Mineralization is dependent on the supplemented concentration of ascorbic acid and $\beta$-glycerol phosphate.

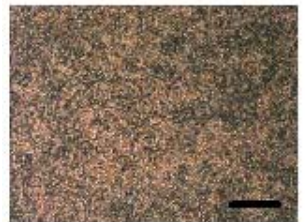

Control

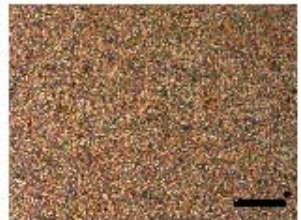

$50 \mu \mathrm{g} / \mathrm{ml}$ AA

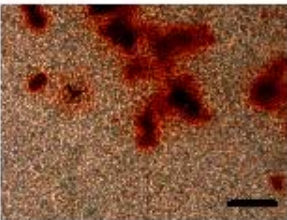

$50 \mu \mathrm{g} / \mathrm{ml} \mathrm{AA}$ $3 \mathrm{nM} \beta-\mathrm{GP}$

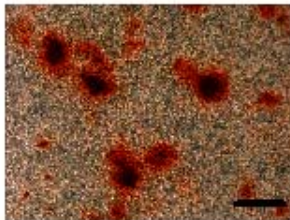

$50 \mu \mathrm{g} / \mathrm{ml} \mathrm{AA}$ 4 пM $\beta-G P$

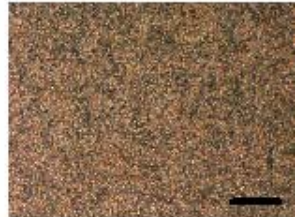

$50 \mu \mathrm{g} / \mathrm{ml} \mathrm{AA}$ $5 \mathrm{nM} \beta-\mathrm{GP}$

Figure 4-5: MC3T3-E1 alizarin red stained cell cultures on Day 15 at 10X magnification. The dark staining, for $50 \mu$ ascorbic acid with 3 and $4 \mathrm{nM}$ of $\beta$-glycerol phosphate, indicates calcium deposits. Bar $=200 \mu \mathrm{m}$. 


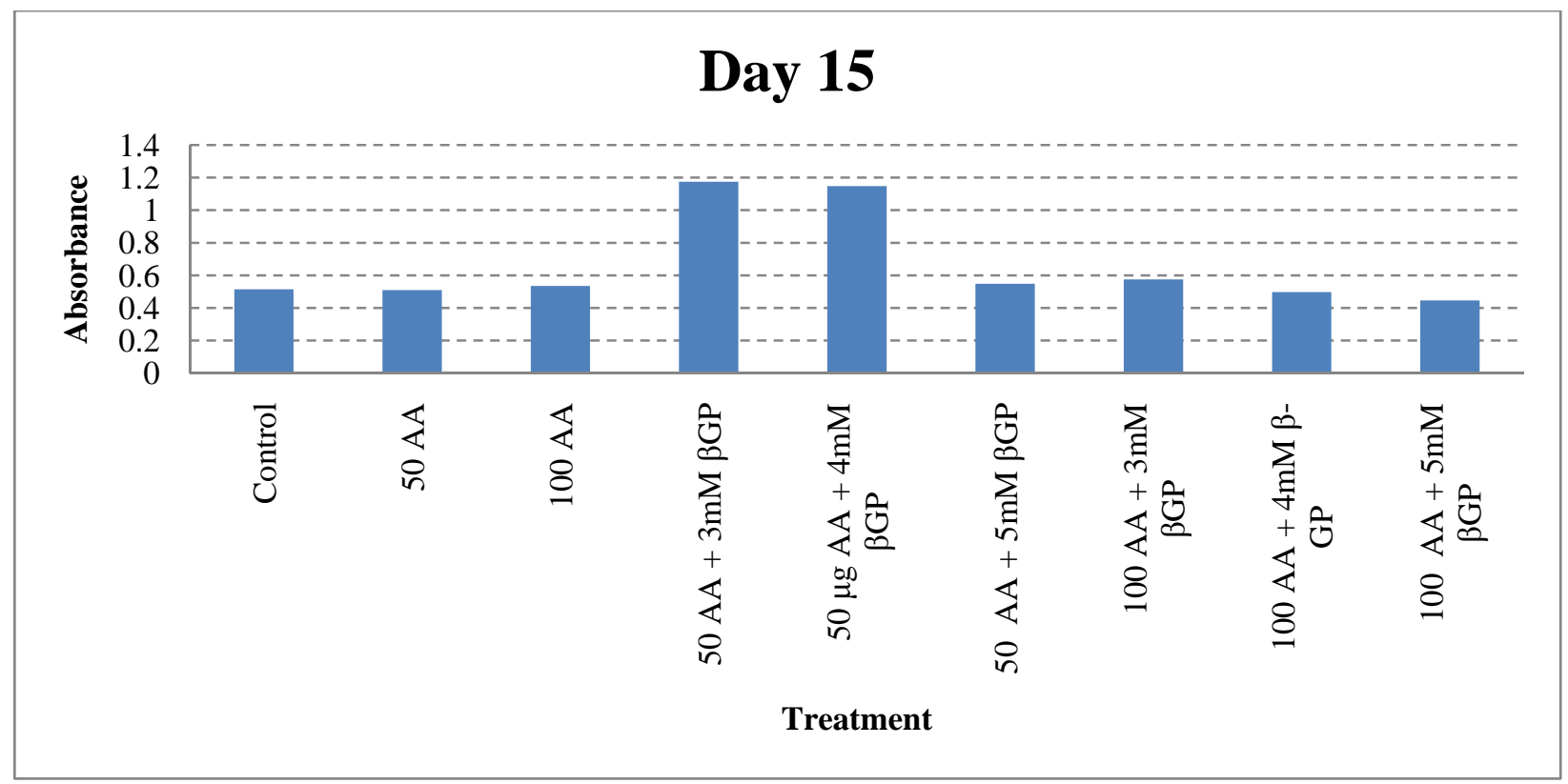

Figure 4-6: Spectrometer measurements of rehydrated alizarin red samples. Absorbance measurements indicated that mineralization is dependent on the supplemented concentration of ascorbic acid and $\beta$-glycerol phosphate.

With the addition of the correct concentration of Differentiation Supplements (DS) of AA and $\beta$-GP, the cells have shown acceptable levels of mineralization by Day 15 . The supplement of $50 \mu \mathrm{g} / \mathrm{ml}$ of AA with 3nM of $\beta$-GP has brought the cell cultures through the initial stages of proliferation and collagen matrix formation to the final stages of differentiation where the collagen matrix has begun to mineralize. Based on the tested variations of AA and of $\beta$-GP, the optimal concentration was found to be $50 \mu \mathrm{g} / \mathrm{ml}$ of AA with 3nM of $\beta-\mathrm{GP}$. For this reason all further experiments were given this differentiation supplement. Going forward the end point of mineralization will be used as confirmation of cell culture differentiation.

\subsubsection{Confirmation of Differentiation}

A set of cell cultures was tested both with and without the cell culture Differentiation Supplement (DS), where DS+ indicates the addition of $50 \mu \mathrm{g} / \mathrm{ml}$ of AA with $3 \mathrm{nM}$ of $\beta$-GP to the 


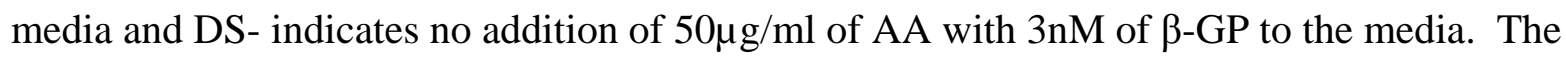
comparison was conducted over 15 days with samples taken at days 5, 10 and 15 (Figure 4-7). The test was run for four different treatment groups, Control (C), LIPUS for 20 minutes (US20), LIPUS for 20 minutes then a 1 hour break then Heat at $40^{\circ} \mathrm{C}$ for 60 minutes (US20 + H60) and Heat for 60 minutes (H60). The treatments were all compared to the Control.

The results show that by day 5 the DS+ cells were statistically significantly more mineralized than the DS- controls (Figure 4-8 - DS+, Figure 4-9 - DS- and Table 4-1). The average absorbance of the DS+ cell cultures was $0.073,0.90$ and 1.00 on days 5, 10 and 15 respectively. The average absorbance of DS- cell cultures was $0.067,0.01$ and 0.01 on days 5, 10 and 15 respectively. Although the differences in optical density are small on day 5, these differences are almost 10 fold by day 10 . The difference in staining between the DS+ and DScultures were apparent to the naked eye by day 10 (Figure 4-7). 


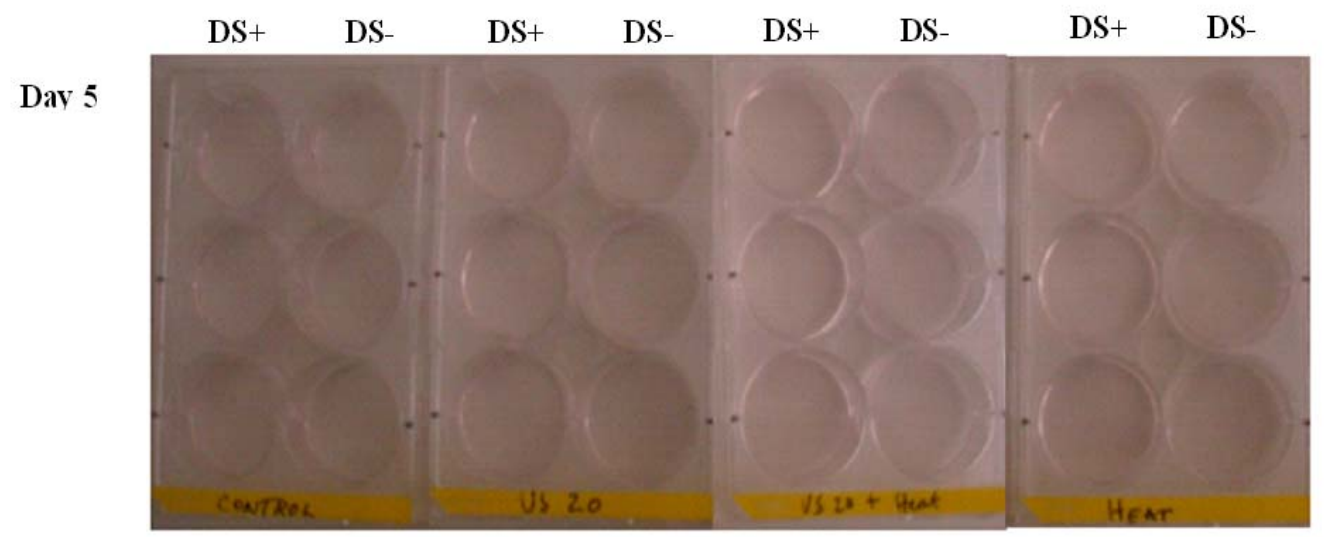

Dav 10

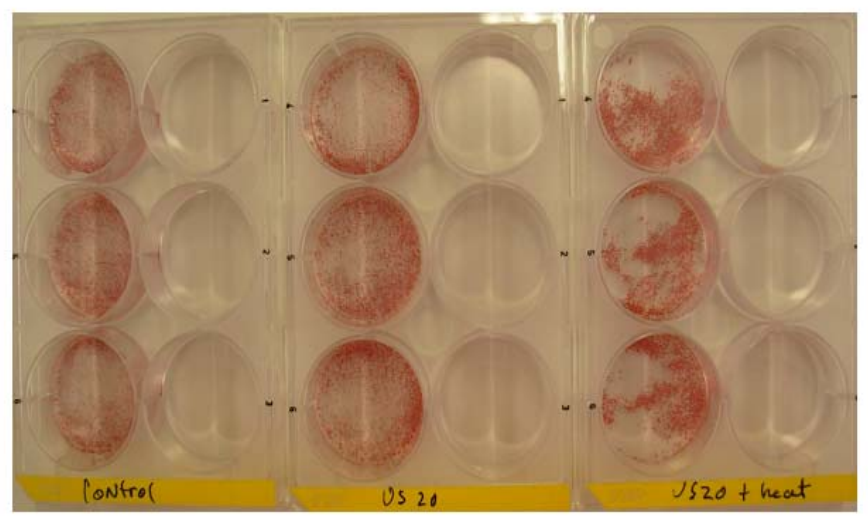

Dav 15

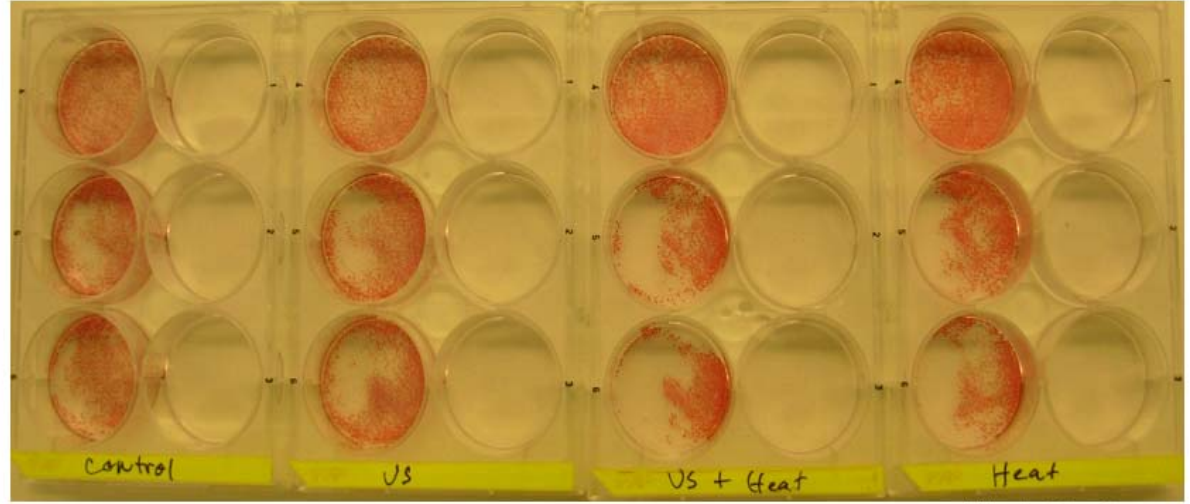

Control

Ultrasound 20

Ultrasound $20 \mathrm{~min}+$

Heat $60 \mathrm{~min}$

Heat $60 \mathrm{~min}$

Figure 4-7: Differentiation supplement positive and differentiation supplement negative cell cultures over time. $(+)$ indicates that differentiation supplements have been added, $(-)$ indicates that differentiation supplements have not been added. Note: The treated culture was contaminated on Day 10. 


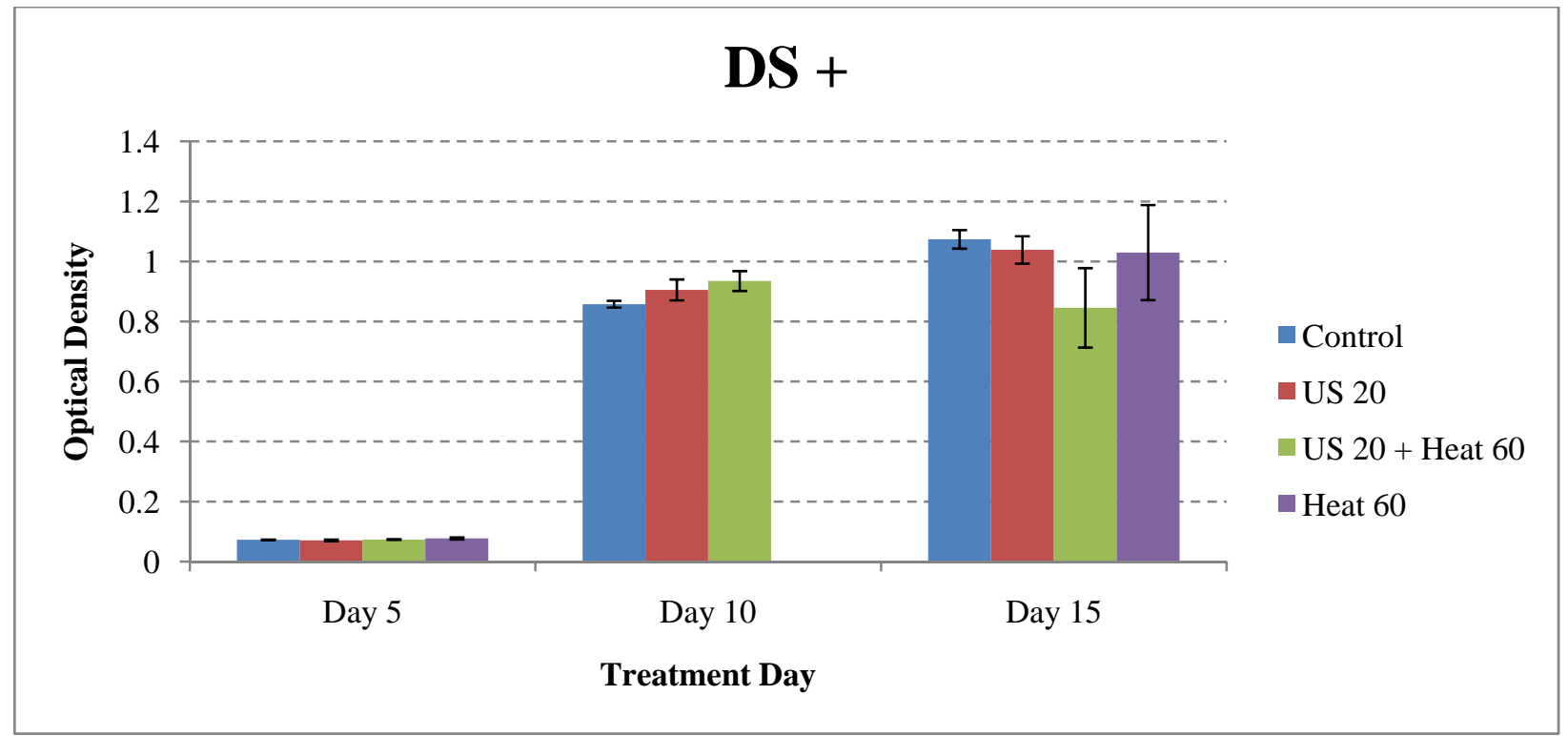

Figure 4-8: Optical density of calcium absorption for differentiation supplement positive cell cultures. The treated culture was contaminated on Day 10. The error bar represents standard error of 3 samples.

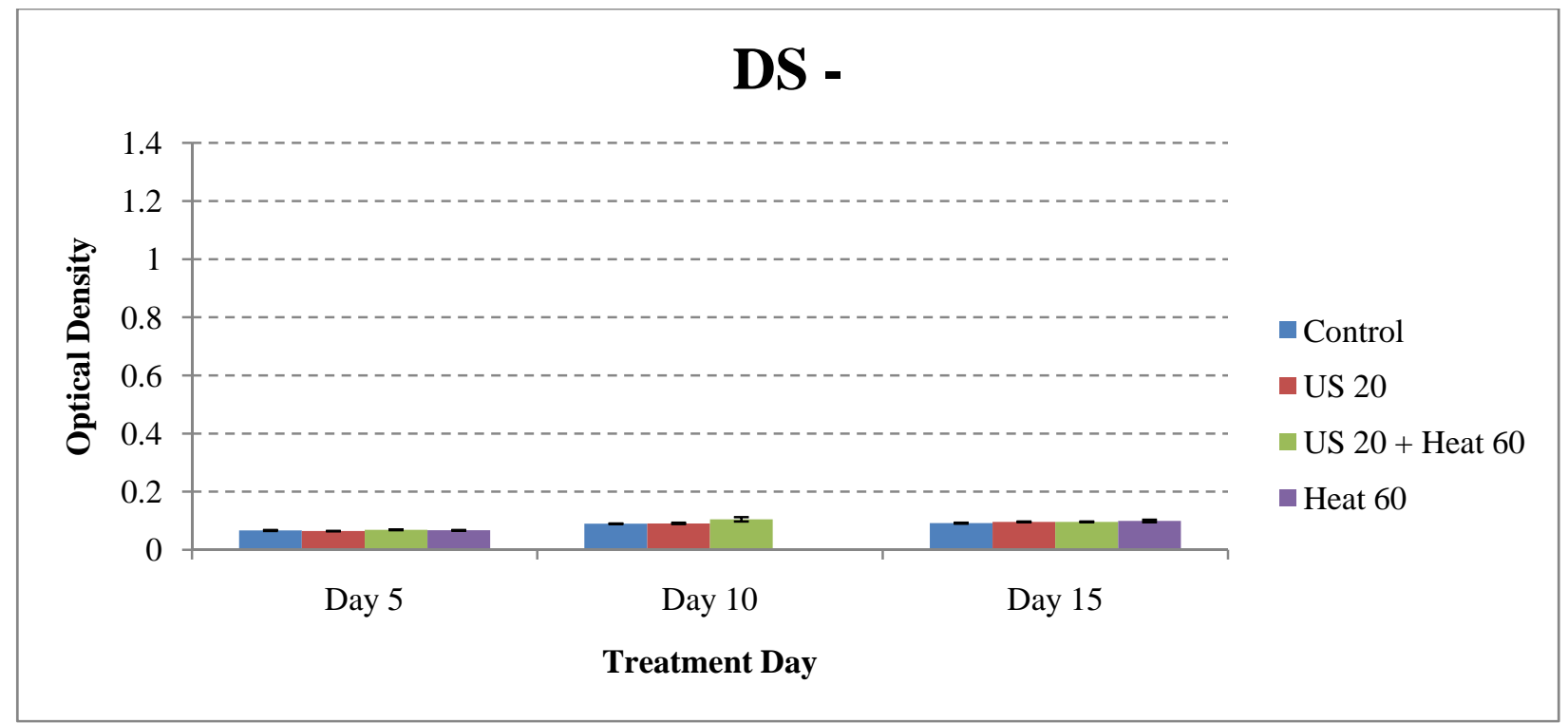

Figure 4-9: Optical density of calcium absorption for differentiation supplement negative cell cultures. The treated culture was contaminated on Day 10. The error bar represents standard error of 3 samples.

Table 4-1, describing the confirmation of differentiation, indicates that DS+ cell cultures have statistically significantly higher mineralization than the DS- cell cultures. These results are 
comparable to published values showing significant differentiation through mineralization. Tang et al (Tang et al 2004) in Figure 4-10 shows that by day 10 DS+ cell cultures appear more mineralized naked eye. Nøregaard et al. (Nørgaard et al 2006) demonstrates that human mesenchymal stem cells supplemented with vitamin D, $\beta$-GP and AA will differentiate into mature osteoblast and undergo a 10 fold increase in mineralization compared to a nonsupplemented controls (Figure 4-20). This trial, for confirmation of differentiation, indicates that the addition of DS+ to cell culture showed statistically significant increases in mineralization.

All the treatment groups showed similar results of differentiation. Table 4-2, describing the treatment effect, indicates that there was no statistically significant difference between treatment groups.

\begin{tabular}{ccccc}
\hline \hline & Control & US20 & US20 + H60 & H60 \\
\hline Day 5 & 0.0261 & 0.0106 & 0.0471 & 0.0481 \\
Day 10 & 0.0001 & 0.0001 & 0.0001 & $\underline{*}$ \\
Day 15 & 0.0001 & 0.0001 & 0.0048 & 0.0042 \\
\hline
\end{tabular}

Table 4-1: Cell Differentiation Statistics - $P$ values. The $P$ value represents the probability that the mean mineralization of the differentiation positive supplements is greater than the differentiation negative supplement within each treatment. Statistical significance was reached by day 5 . P values less than 0.05 are considered statistically significant. (*)The treated culture was contaminated on Day 10.

\begin{tabular}{cccc}
\hline \hline & US20 & US20 + H60 & H60 \\
\hline Day 5 & 0.6218 & 0.5991 & 0.2889 \\
Day 10 & 0.2654 & 0.0919 & $\underline{*}$ \\
Day 15 & 0.5577 & 0.1686 & 0.7983
\end{tabular}

Table 4-2: Treatment Effect Statistics $-P$ values. The $P$ value represents probability that the mean mineralization of the Treatment DS+ treatments is greater than the Control DS+ treatment. P values less than $\mathbf{0 . 0 5}$ are considered statistically significant. (*)The treated culture was contaminated on Day 10. 


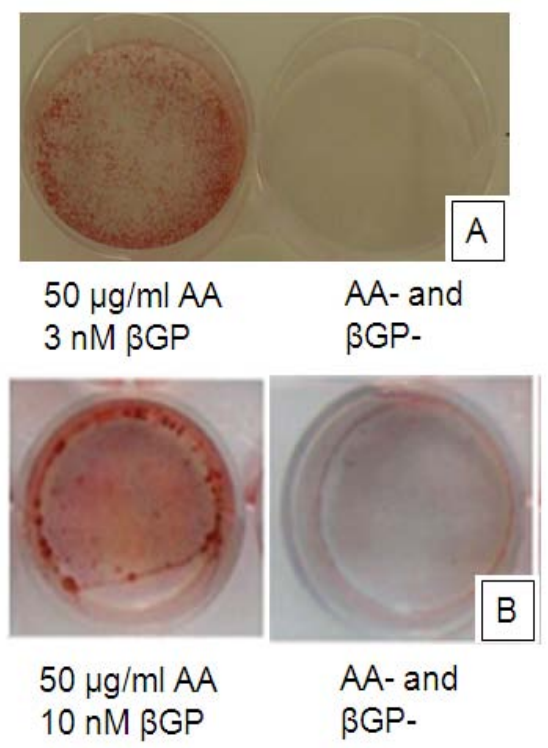

Figure 4-10: Cell culture differentiation shown positive staining for calcification. A) DS+ cell culture - the alizarin red has bonded with the calcium expressed by the cells. B) Previously published results show alizarin red has bonded with calcium expressed by the cells (Tang et al 2004).

\subsection{Ultrasound Treatment Effect - Comparison to Published Results}

A trial was run to compare the results from this lab with previously published results (Unsworth et al 2007), and ensure the cell and equipment were working properly. The trial consisted of two treatment groups; LIPUS for 20 minutes (US20) and Control (C). The trial was implemented using a single passage of cells. Samples were assessed on days 5, 10 and 15 (Figure 4-13 and Figure 4-14). On days 10 and 15 differentiated wells showed statistically significantly higher optical absorption than non differentiated wells (Figure 4-11 and Table 4-3). The statistically significant difference suggests that there is higher mineralization in the DS+ cells.

Although the color difference between the treatment groups was small on days 10 and 15, the apparent mineralization was statistically significantly higher than the controls (Figure 4-12 
and Table 4-4). In addition, day 10 ultrasound and day 15 ultrasound were not significantly different from each other. The differences in mineralization seem small compared to those measured in the previous experiment (section 4.1.3) however they are comparable to previously published works (Figure 1-8). The treatment of cells with LIPUS at 20 minutes/day seemed to have a statistically significant effect by day 10 .

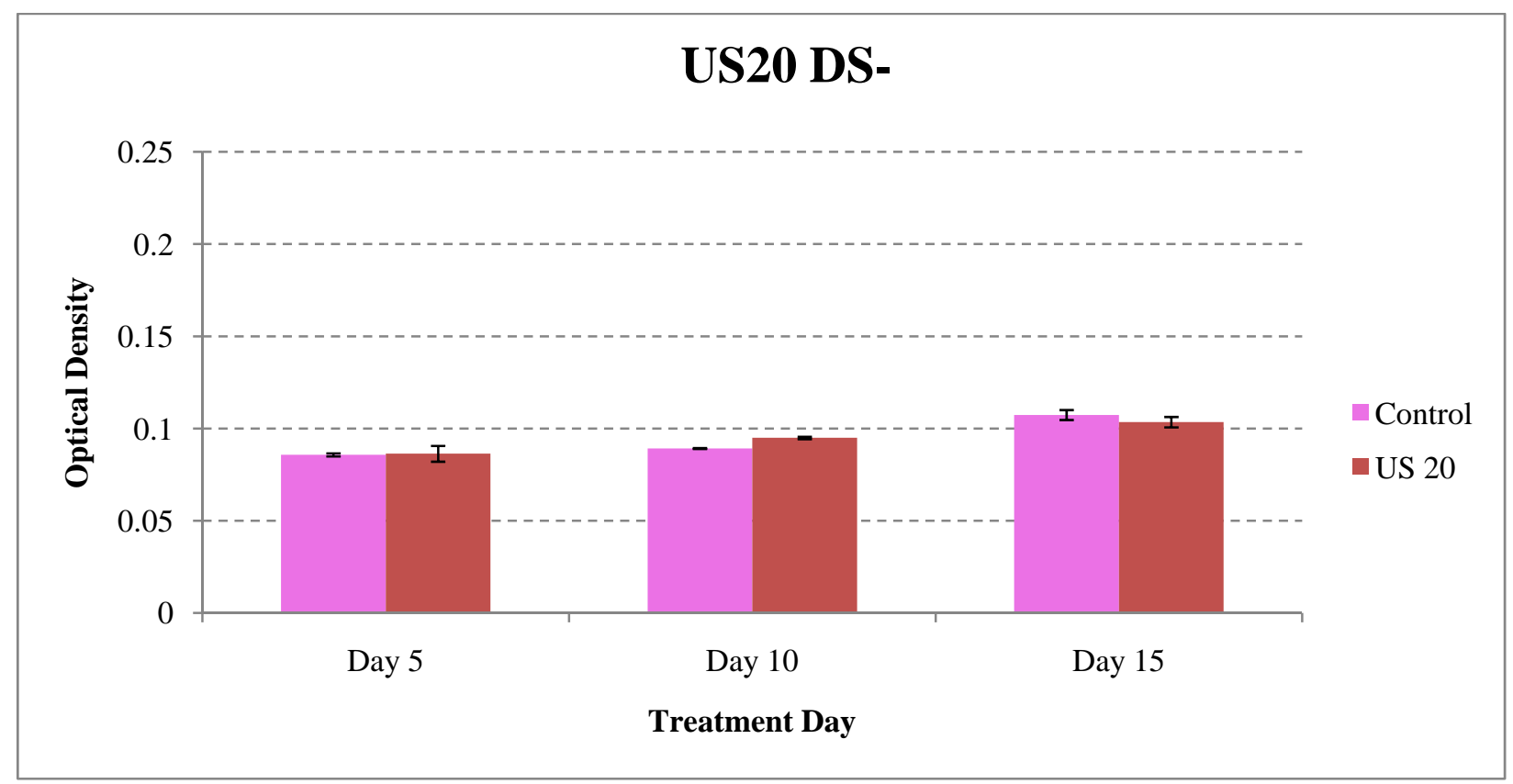

Figure 4-11: Comparison of US20 treatment to Control treatment for differentiation supplement negative samples. Error bar indicated the standard error of 12 measurements.

\begin{tabular}{ccc}
\hline \hline & Control & US20 \\
\hline Day 5 & 0.6514 & 0.4446 \\
Day 10 & 0.0001 & 0.0475 \\
Day 15 & 0.0478 & 0.0012 \\
\hline
\end{tabular}

Table 4-3: Cell Culture Differentiation Statistics - $P$ values. The $P$ value represents the probability that the mean mineralization of DS+ cell culture is greater than the DS- cell culture in the same treatment group. Statistical difference reached by day 10. $P$ values less than 0.05 are considered statistically significant. 


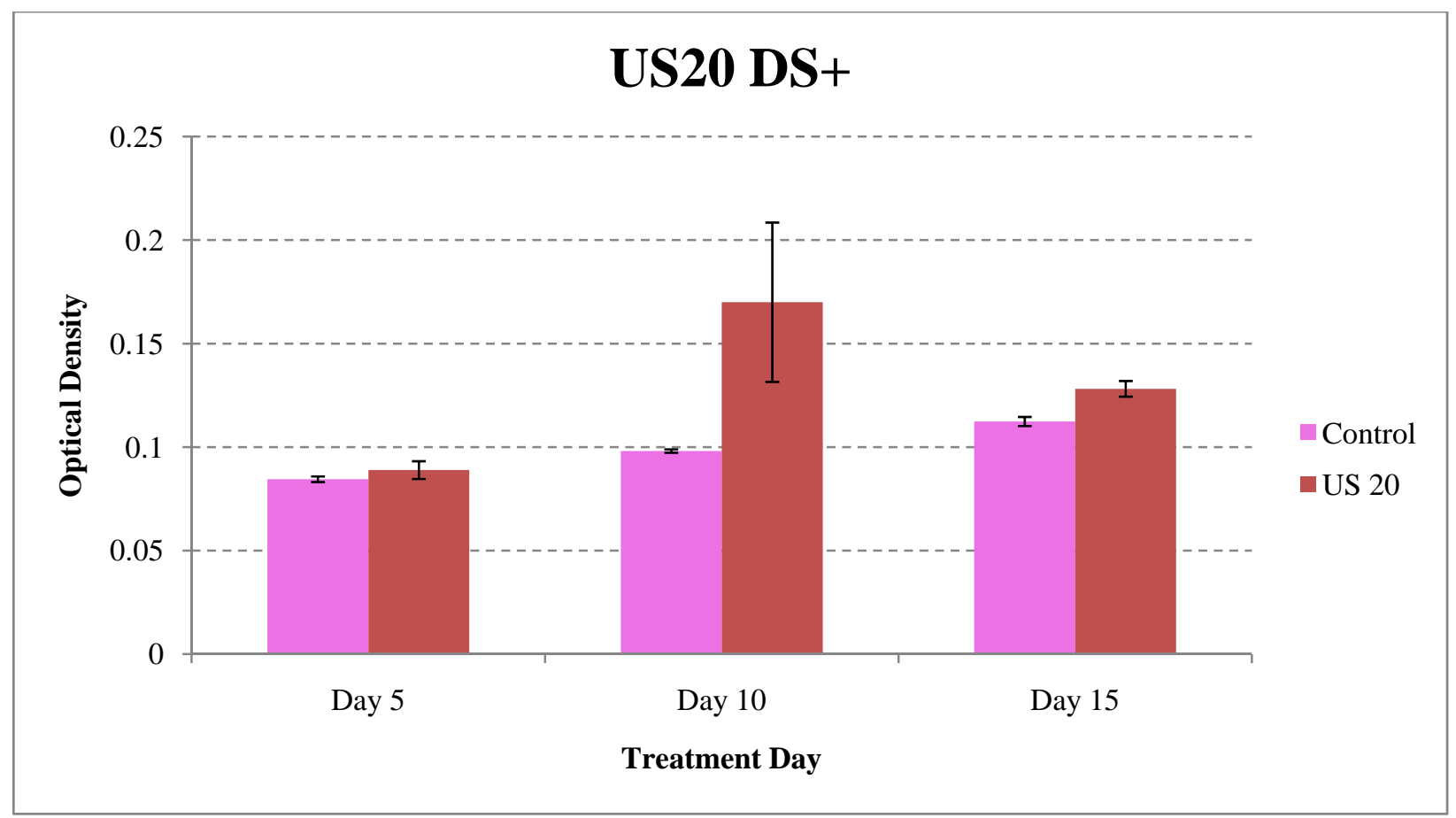

Figure 4-12: Comparison of US20 treatment to Control treatment for differentiation supplement positive samples. Error bar indicated the standard error of 12 measurements.

\section{US20}

Day 5

0.0669

Day 10

0.0074

Day 15

0.0022

Table 4-4: Treatment Effect Statistics - $P$ values. The $P$ value represents the probability that the mean mineralization of the treatment is greater than that of the control. Statistical difference reached by day 10.P values less than 0.05 are considered statistically significant. 
Ultrasound 20

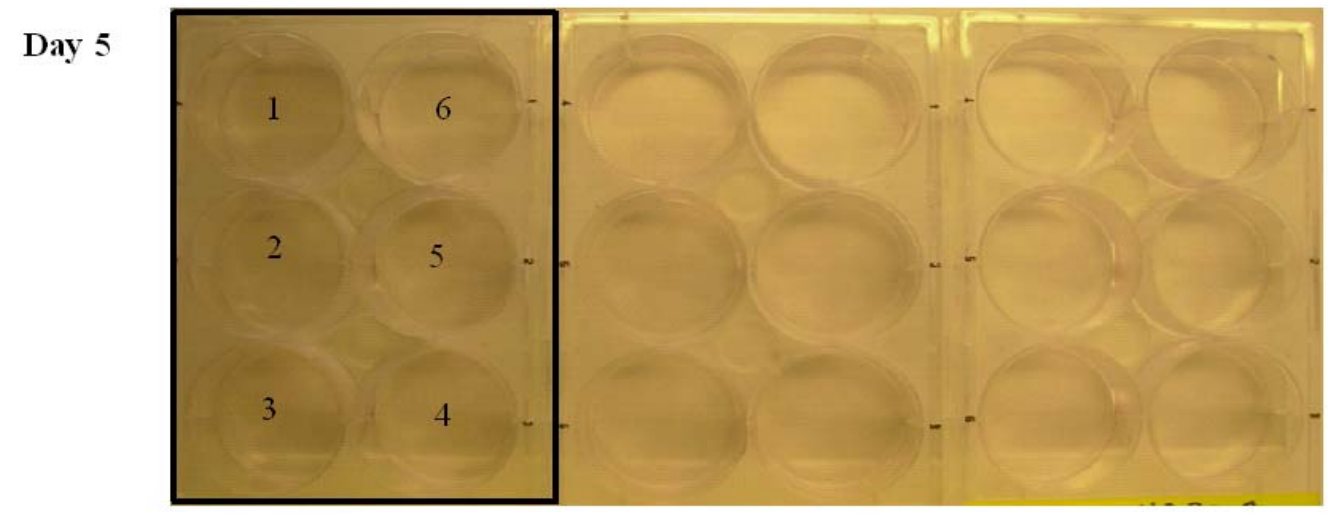

Day 10

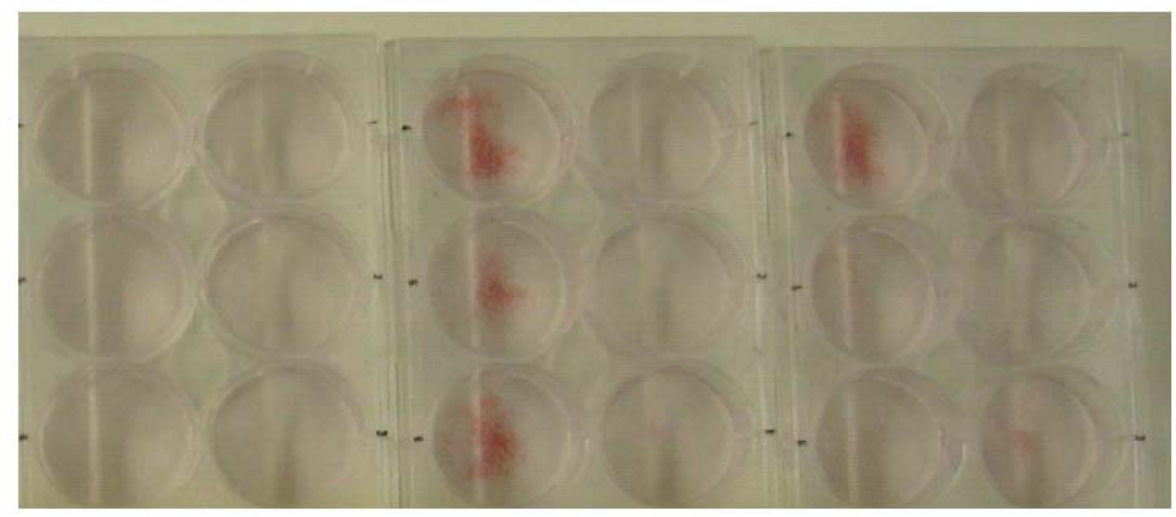

Day 15

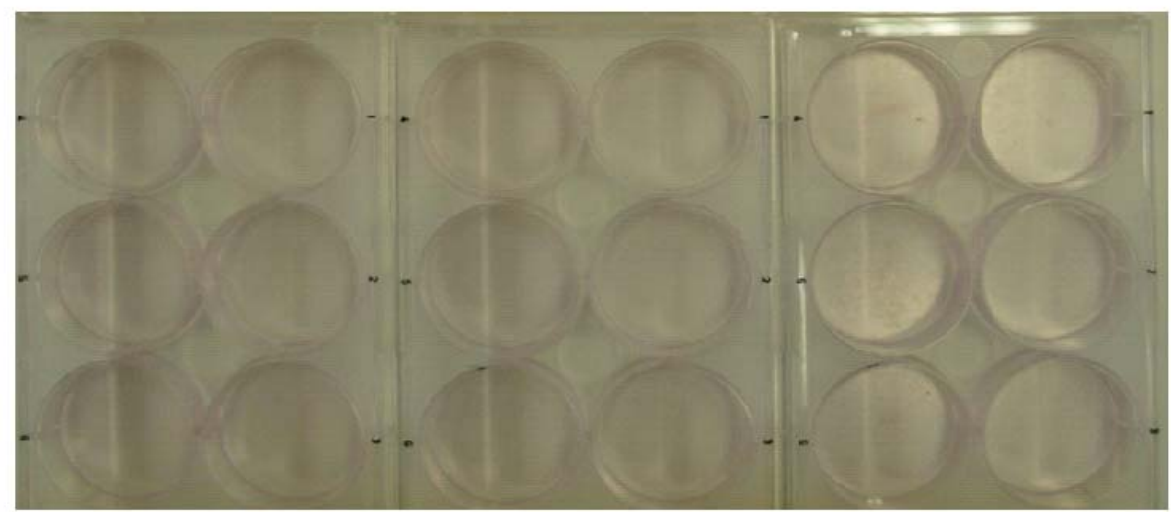

Figure 4-13: Ultrasound 20 minute treated cell cultures over 15 days. Positions 1-4 are DS+ and Positions 5-6 are DS- for all well plates. 


\section{Control}

Day 5
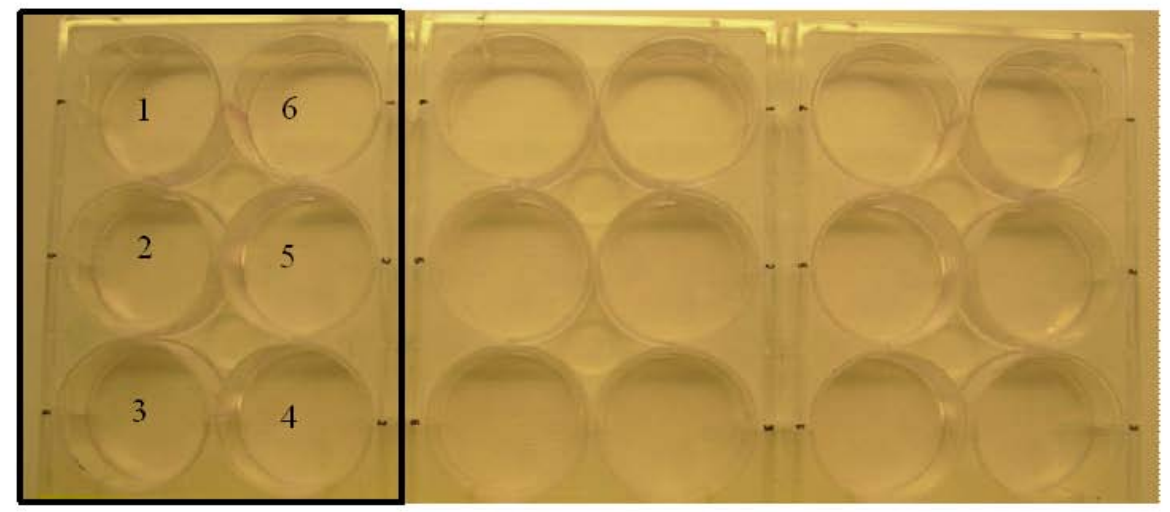

Day 10

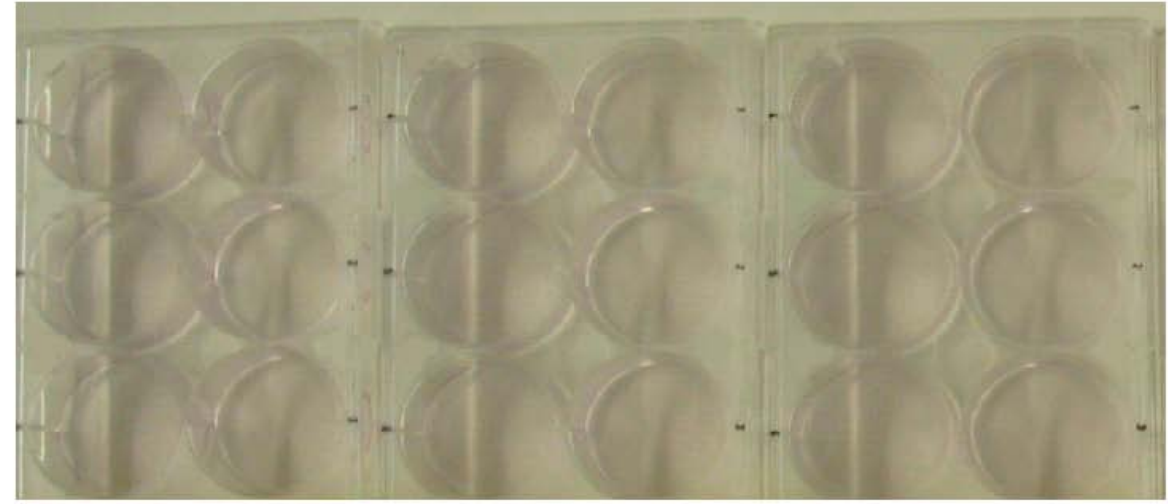

Day 15

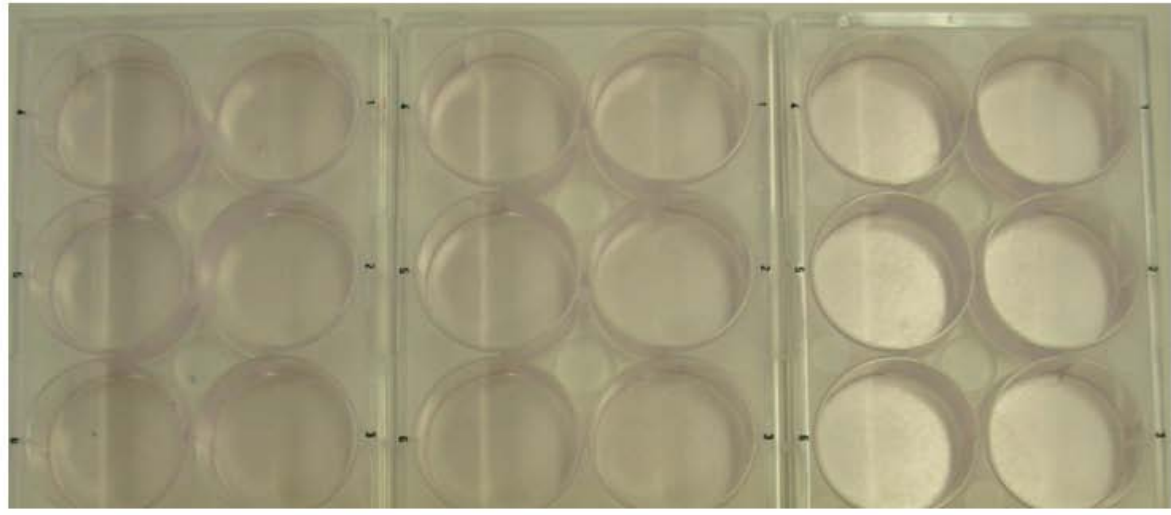

Figure 4-14: Control treated cell cultures over 15 days. Positions 1-4 are DS+ and Positions 5-6 are DS- for all well plates. 


\subsection{Results - Test of Hypothesis - Combined Treatment effect}

When testing LIPUS against the control as shown in section 4.2, the results showed much weaker mineralization than the experiment described in the section 4.1.3. In an effort to increase mineralization some the treatments were adjusted. This trial consisted of four treatment groups; Control (C), LIPUS for 40 minutes (US40), LIPUS for 40 minutes plus Heat for 40 minutes concurrently (US40 + H40), and Heat for 40 minutes (H40). In addition, the experiment was repeated 3 times to account for possible effects due to variations in seeding and cell passage number. The cells samples were taken from passages 4,5 and 6 .

By the $5^{\text {th }}$ day after treatment, all cell groups showed significant differentiation, when measured against day 0 cells (Figure 4-15). This means that there was a greater degree of mineralization on day 5 than there was on day 0 . This was true of all cell culture treatment groups over all three trials.

By day 15, Table 4-5 indicates that the mean optical absorbance of US40 and US40 + H40 has increased almost 6 fold over the Control and H60 samples. H40 showed an increase in mineralization of 1.2 fold over the control, which is comparable to published values (Figure 4-20) (Nørgaard et al 2006). By this time all treatment groups were statistically significantly improved over the Control treatment group (Figure 4-15 and Table 4-6). The results show that US40, US40 + H40, and H40 treatment groups all statistically significantly improved mineralization when compared to the Control. The error bars for the US40 and US40 + H40 treatment groups were much larger than the error for the $\mathrm{H} 40$ treatment group and the $\mathrm{C}$ treatment group. In addition, there was no statistically significant difference in mineralization between the US40 and the US40 + H40 treatments. 
When the treatments are compared within each group, it is clear that there is an increase in mineralization over time (Figure 4-16). Both of the LIPUS and the LIPUS + heat treatment groups showed that distinct mineralization starts between days 10 and 15 . This trend indicates that mineralization seems to begin in this window of time.

\begin{tabular}{lcccc}
\hline \hline & Control & US40 & US40 + H40 & H40 \\
\hline Day 5 & 0.116 & 0.107 & 0.111 & 0.109 \\
Day 10 & 0.140 & 0.159 & 0.158 & 0.127 \\
Day 15 & 0.153 & 0.606 & 0.618 & 0.186 \\
\hline
\end{tabular}

Table 4-5: Mean optical absorbance of treatment samples.

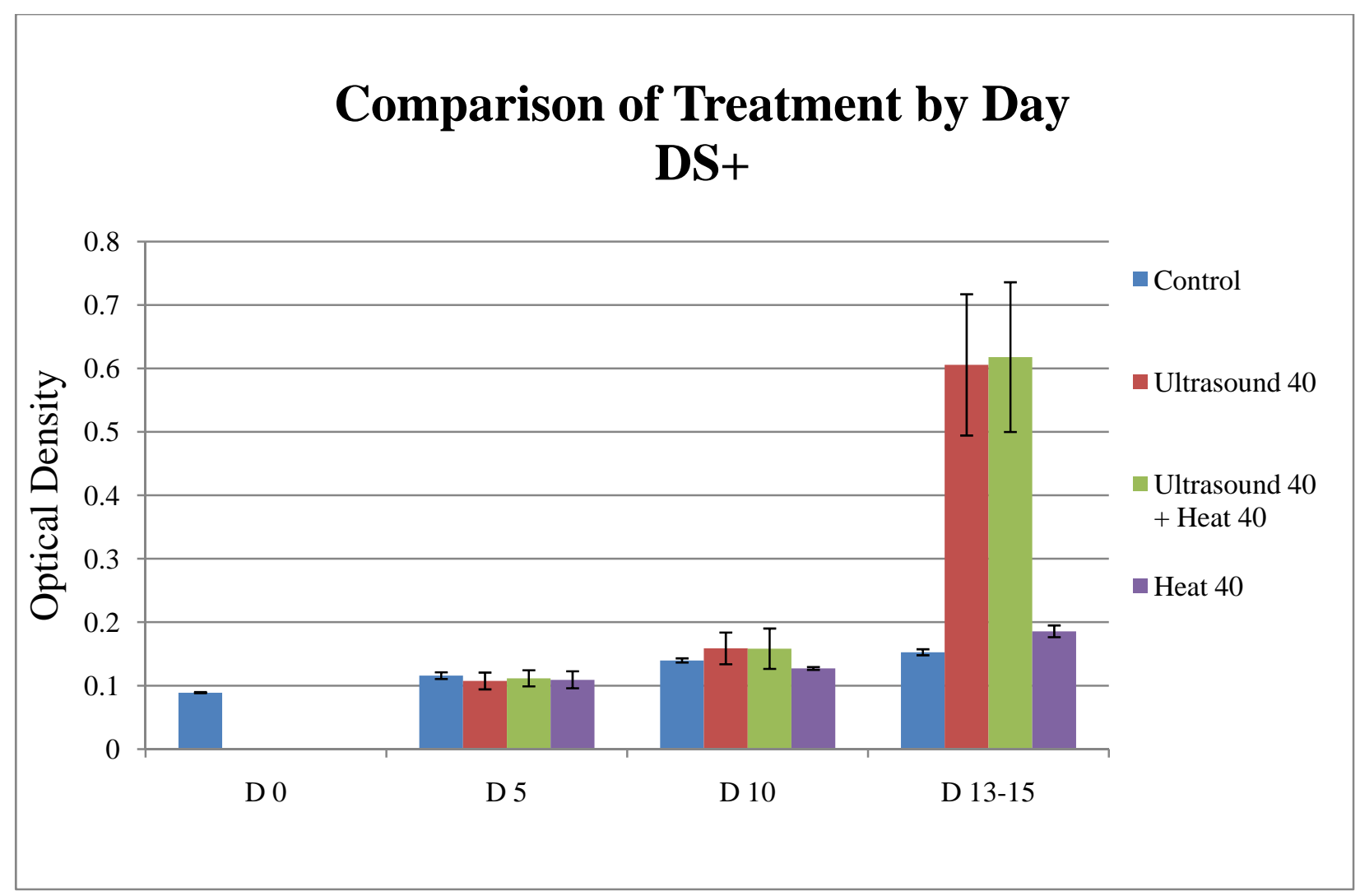

Figure 4-15: Comparison of treatment groups by treatment day. All samples are differentiation supplement positive. Error bar indicates a standard error of 18 measurements. 


\begin{tabular}{cccc}
\hline \hline & US40 & US40 + H40 & H40 \\
\hline Day 5 & 0.0554 & 0.3019 & 0.13 \\
Day 10 & 0.457 & 0.567 & 0.0034 \\
Day 15 & 0.0003 & 0.0004 & 0.0031 \\
\hline
\end{tabular}

Table 4-6: Treatment Effect Statistics - $P$ values. The $P$ value represents the probability that the mean mineralization of the treatment is greater than that of the control. All day 5 measurements are statistically significantly greater than day $0(P=0.0001) . \quad P<0.05$ is statistically significant.

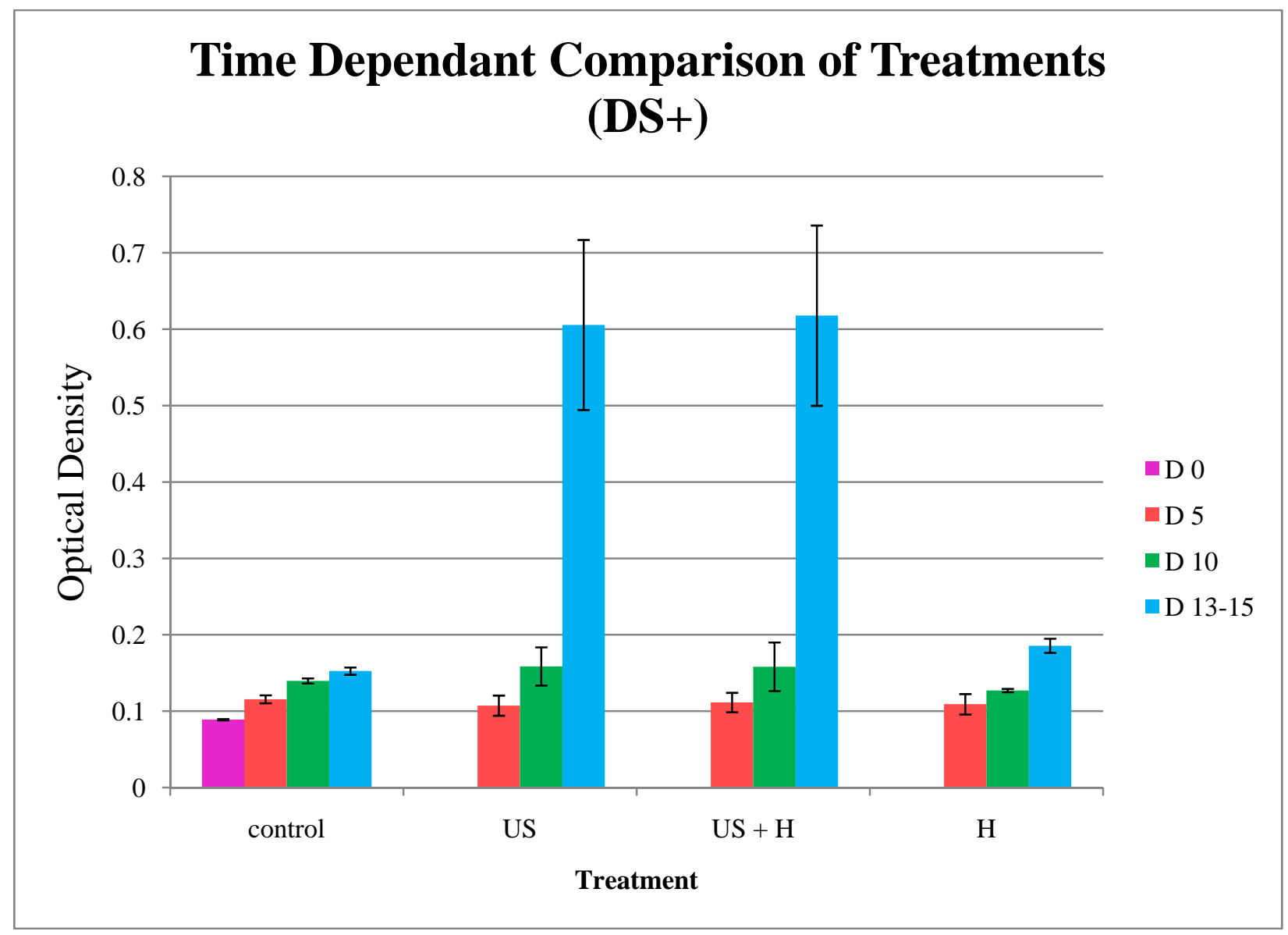

Figure 4-16: Comparison of treatments over time. Error bar indicates a standard error of 18 measurements. 

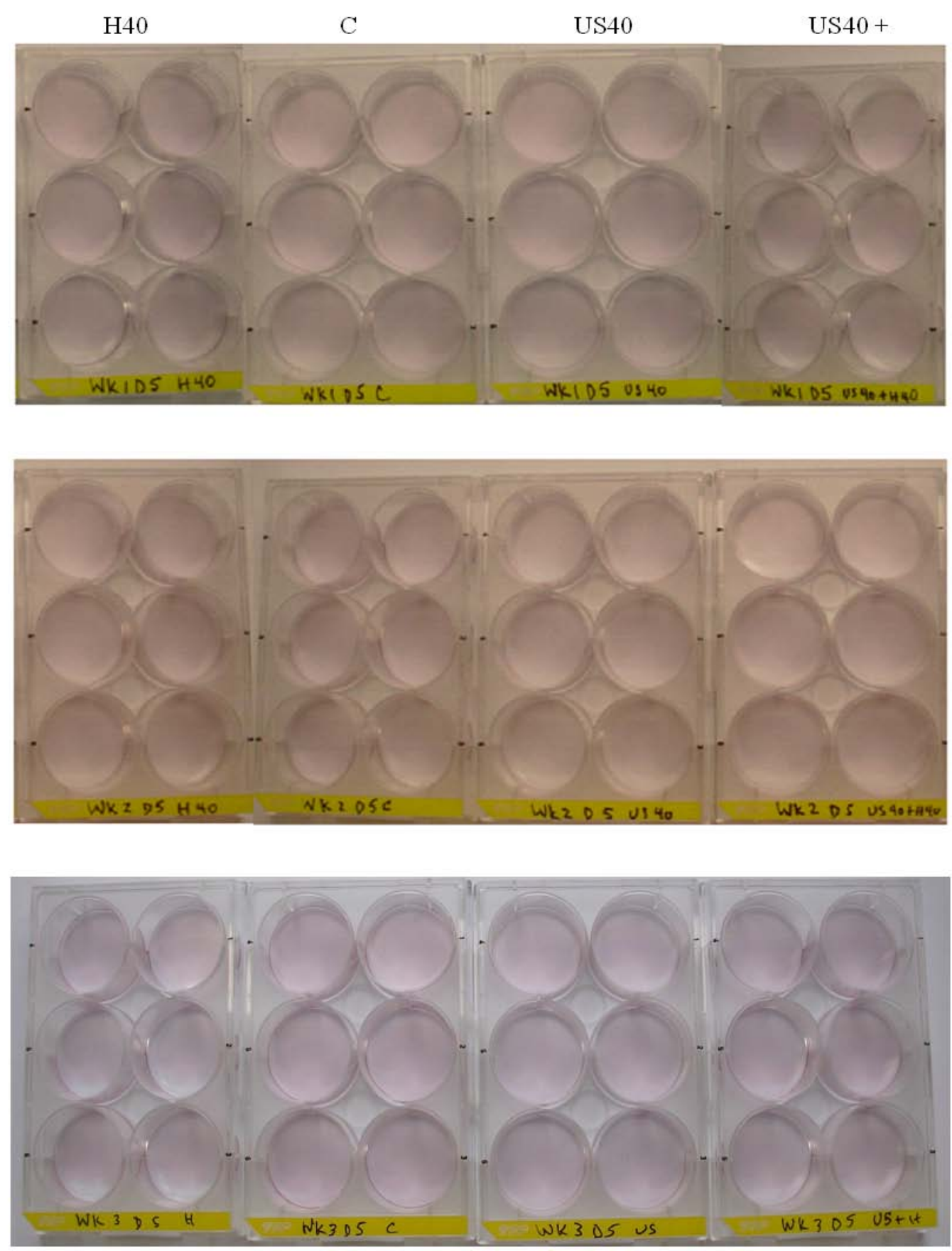

Figure 4-17: All treatment groups day 5. No apparent mineralization. 

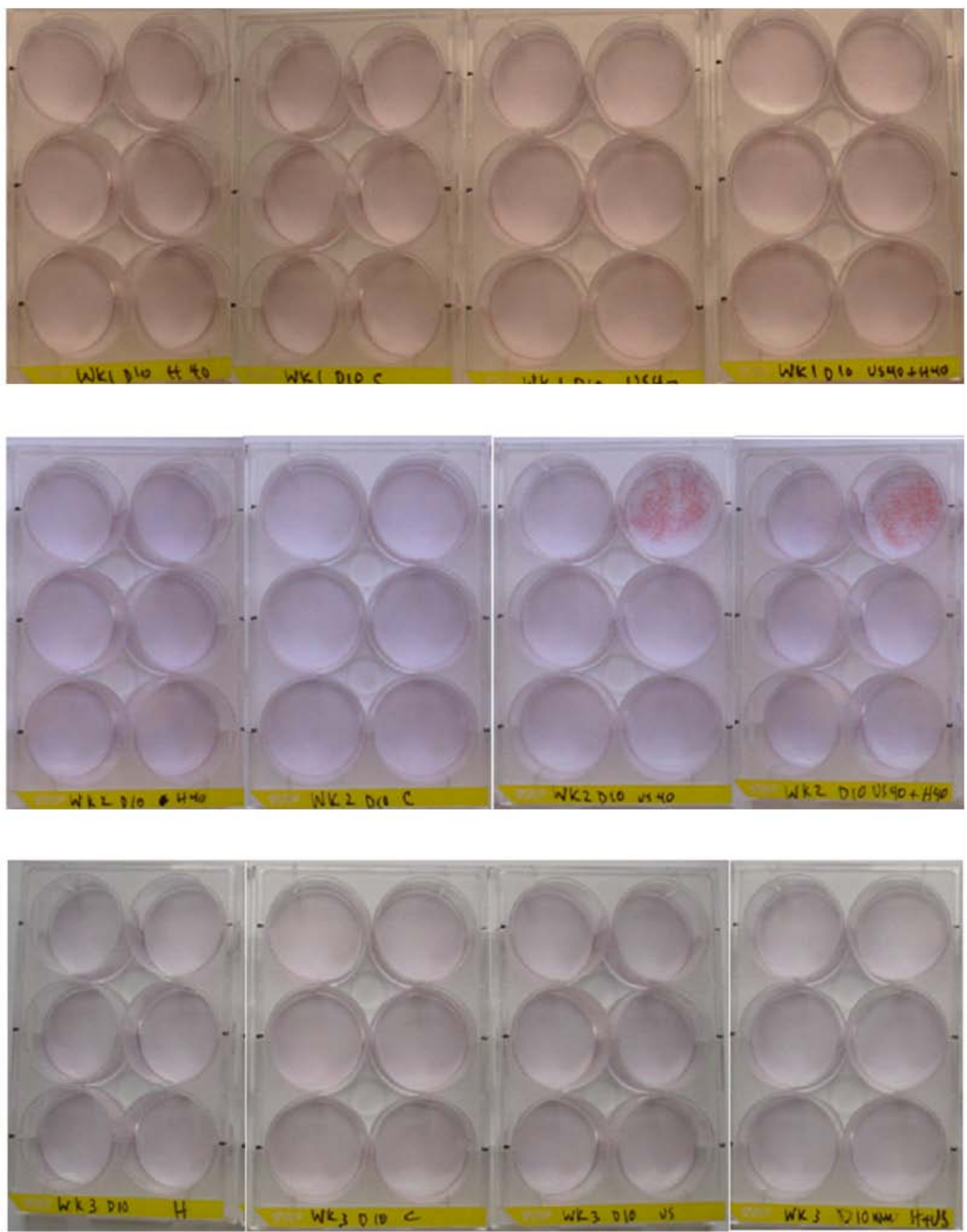

Figure 4-18: All treatment groups day 10. Mineralization apparent only on 1 well for US40 and 1 for US40 + H40 apparent mineralization. 

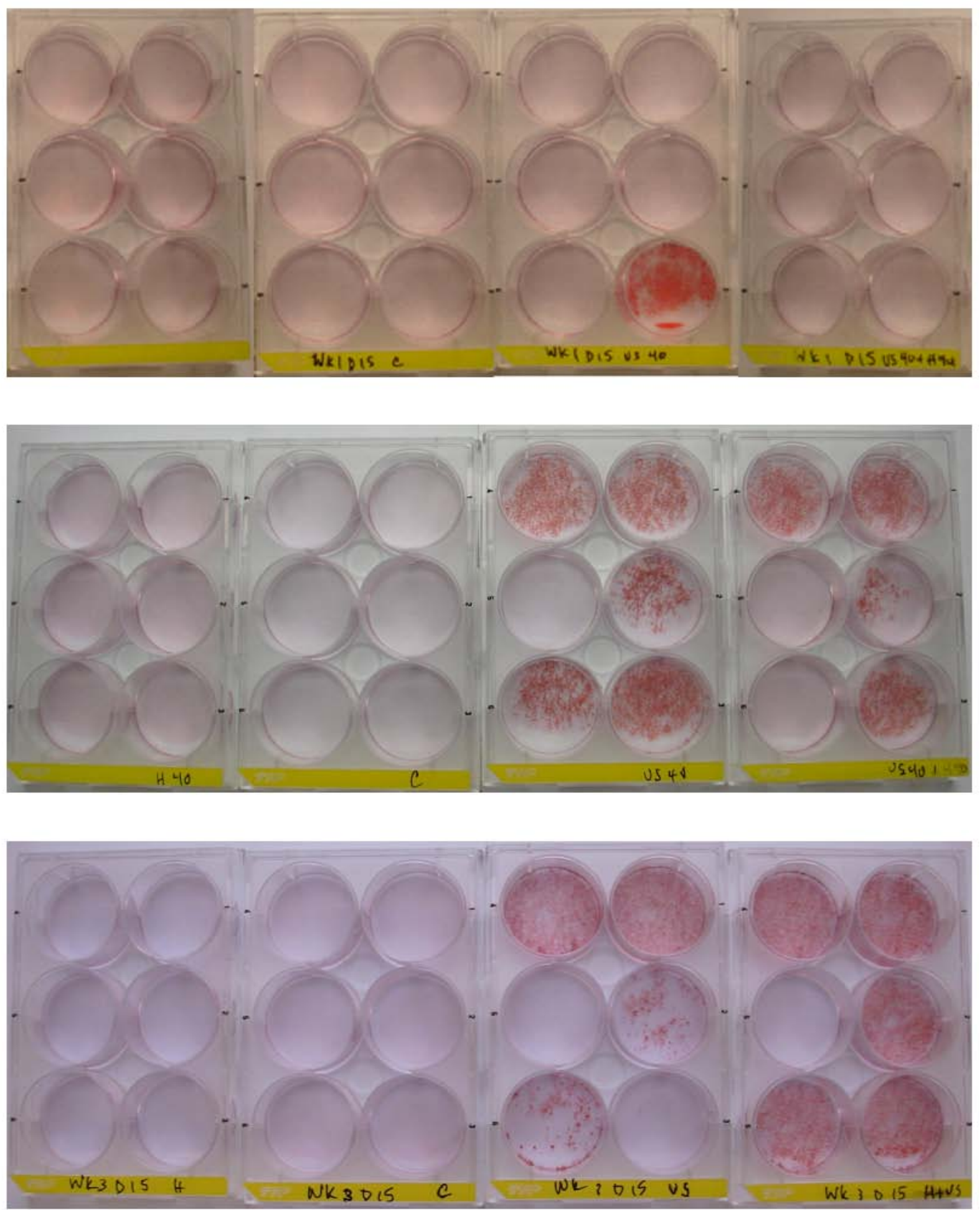

Figure 4-19: All treatment groups day 15. Mineralization apparent on several wells for US40 and US40 + H40 apparent mineralization. 


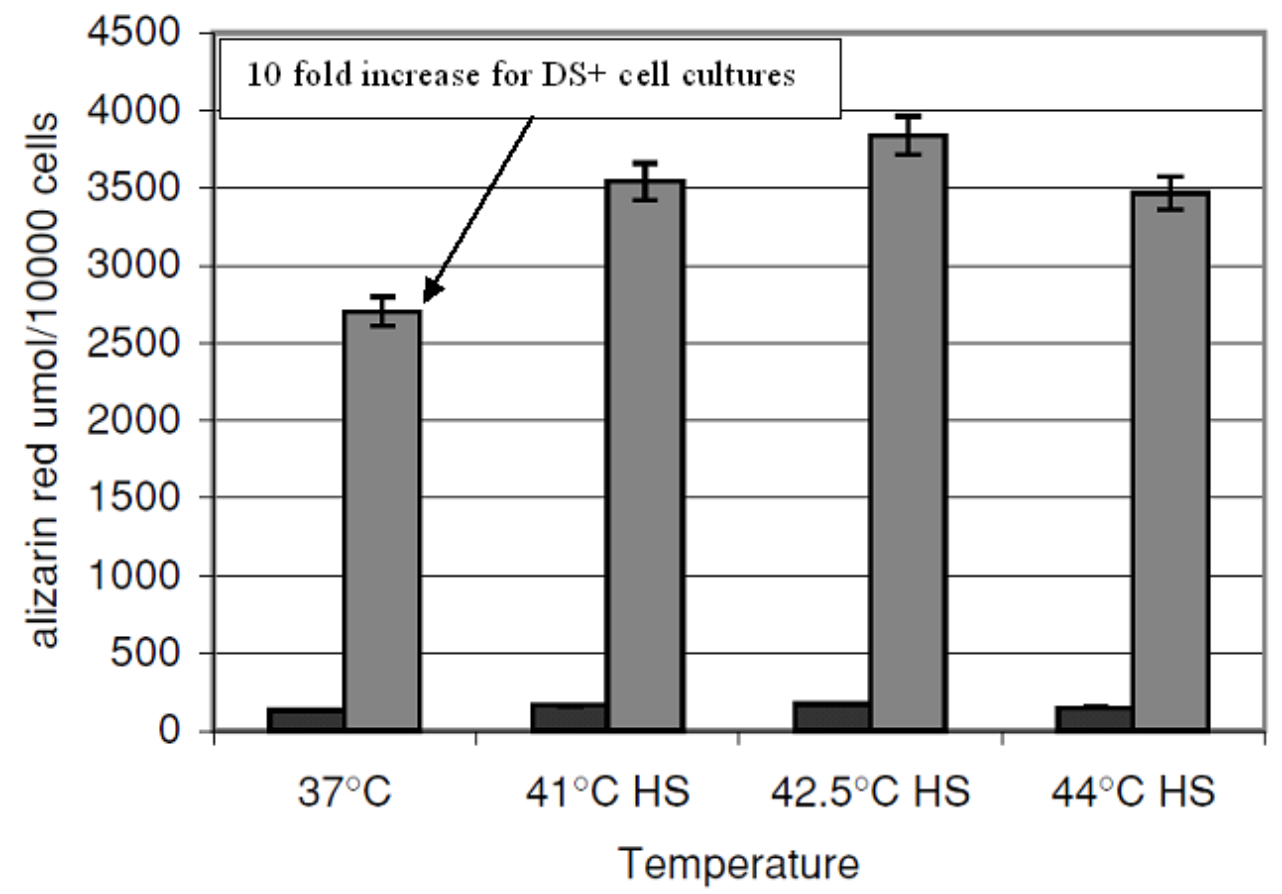

Figure 4-20: Effect of heat shock on mineralized matrix formation for human mesenchymal stem cells. Light grey indicates differentiation supplement positive, dark grey indicates differentiation supplement negative. The supplement to induce differentiation includes vitamin $D, \beta$-glycerol phosphate and ascorbic acid (Nørgaard et al 2006).

\subsection{Spectrum measurements of Alizarin Red}

The expected peak optical absorbance of alizarin red is $420 \mathrm{~nm}$ (Csányi 1980) (Figure 4-21). In processing the cell culture to measure mineralization, chemicals were added that may distort the spectral peak of alizarin red. To determine the effect of each additive on optical absorbance, solutions with different additives were mixed. Filtered water was used as a base line, and then each cell processing chemical was added to the water to assess the effect on the optical peak. The spectral analysis of water, alizarin red and perchloric acid (Figure 4-22, blue) shows that the peak is in the same range as that of previously published work (Figure 4-21). The shape of the spectrum changes when formalin is added to the sham solution. Below $390 \mathrm{~nm}$ there is a spike in the absorbance and between $421-424 \mathrm{~nm}$ there is a jump in the absorbance 
(Figure 4-22, red). When the solution containing formalin is compared to solutions made from cell cultures, the shape of the spectrum is the same (Figure 4-23). Measurements using a plate reader are made at $405 \mathrm{~nm}$. At $405 \mathrm{~nm}$ there is separation between the spectral curves, and the curves have the same shape (Figure 4-24). This indicates that it is unlikely that there are additional particles (like cells) in the solution that are highly absorbent at this wave length.

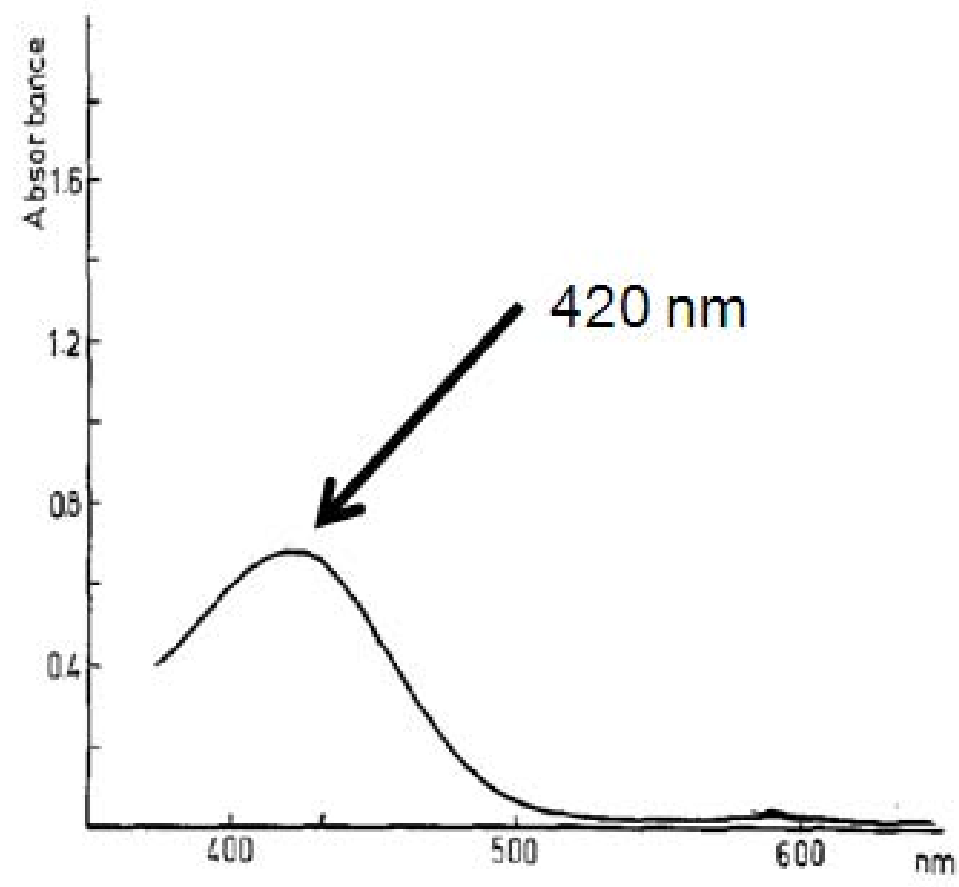

Figure 4-21: 0.5mM Alizarin Red at pH 3.90. The peak occurs at 420 nm. (Csányi 1980) 


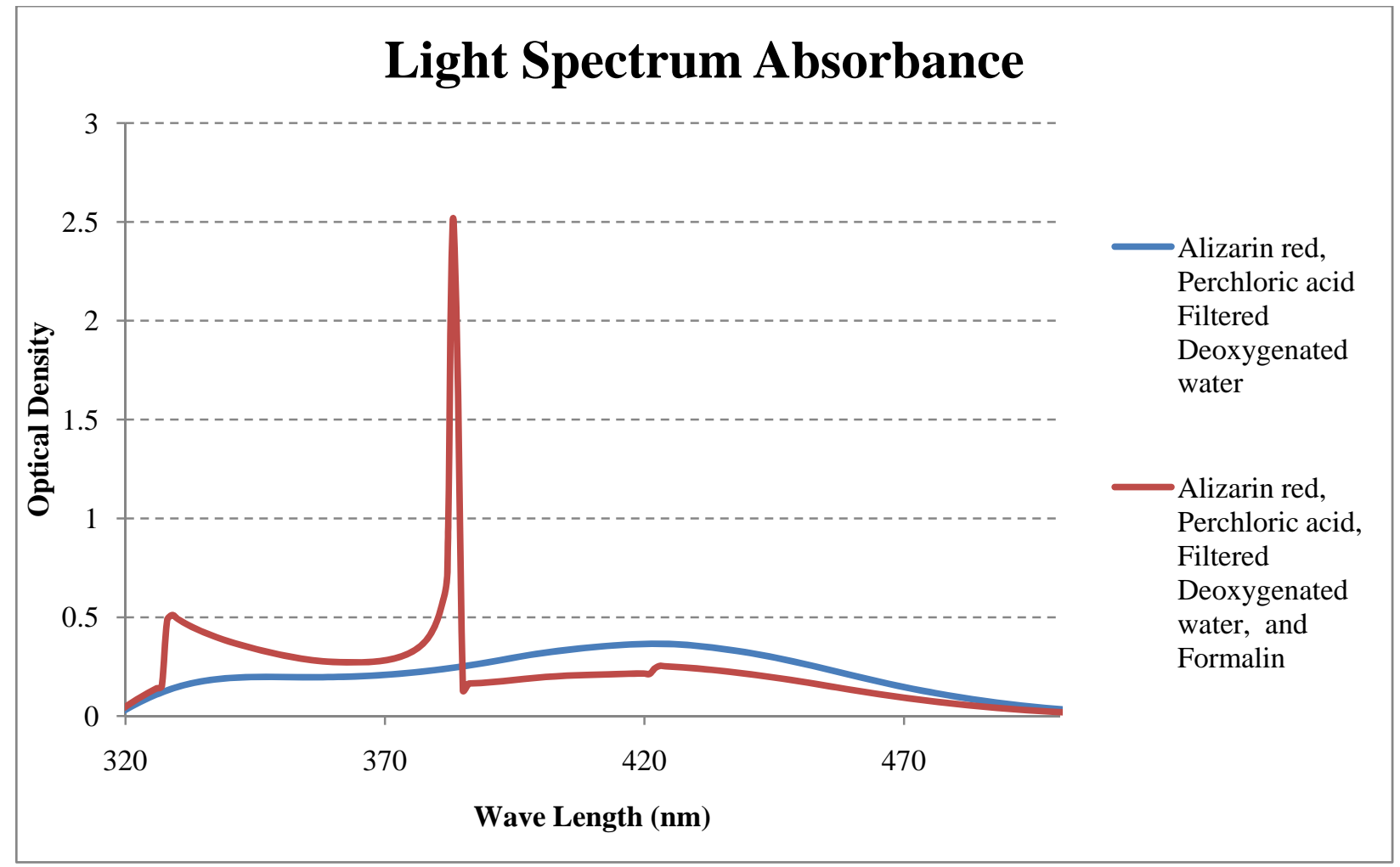

Figure 4-22: Comparison of spectral curves with and without formalin

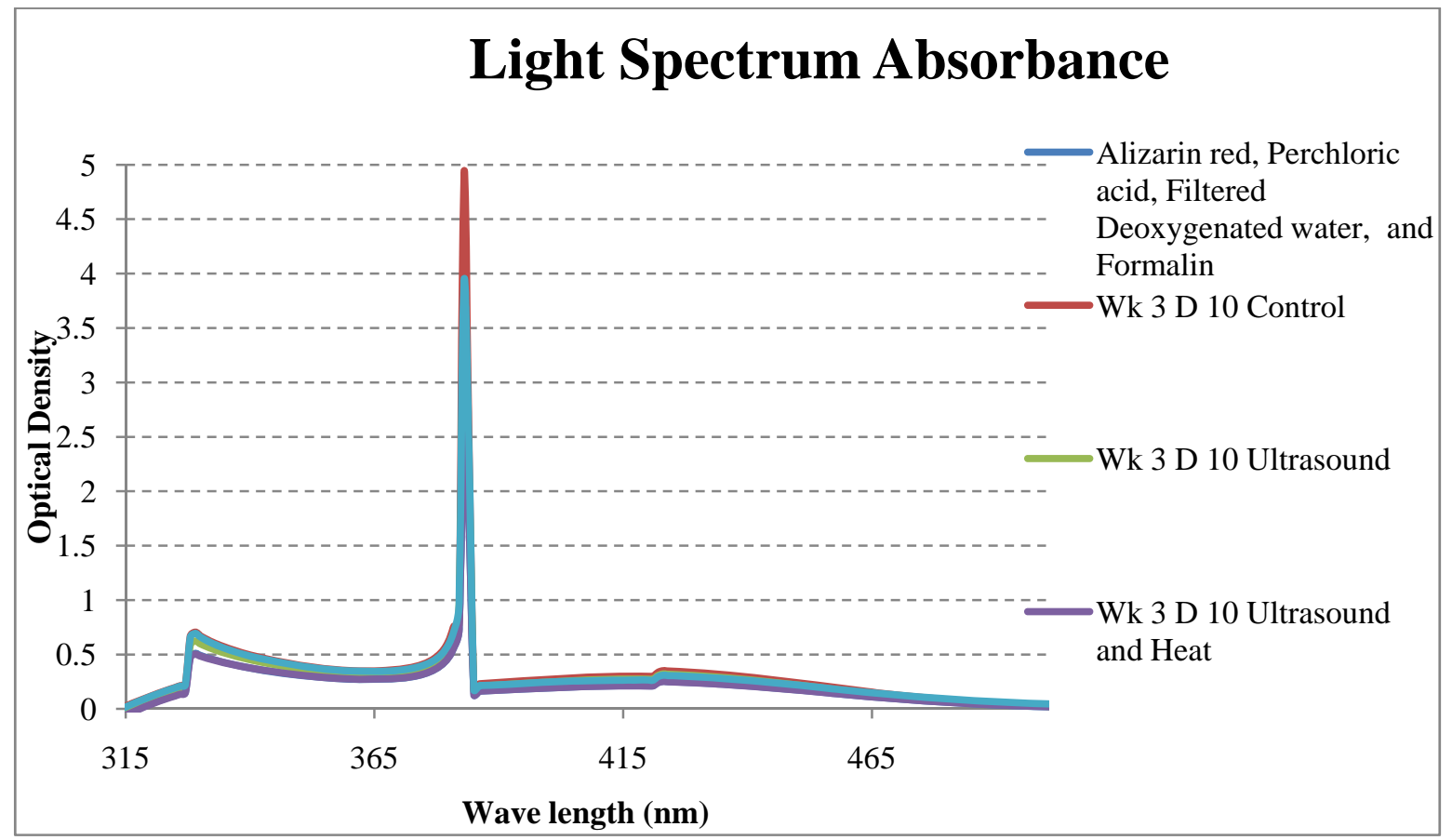

Figure 4-23: Comparison of spectral curves from $315-500 \mathrm{~nm}$. The shape of the spectral curves is similar. 


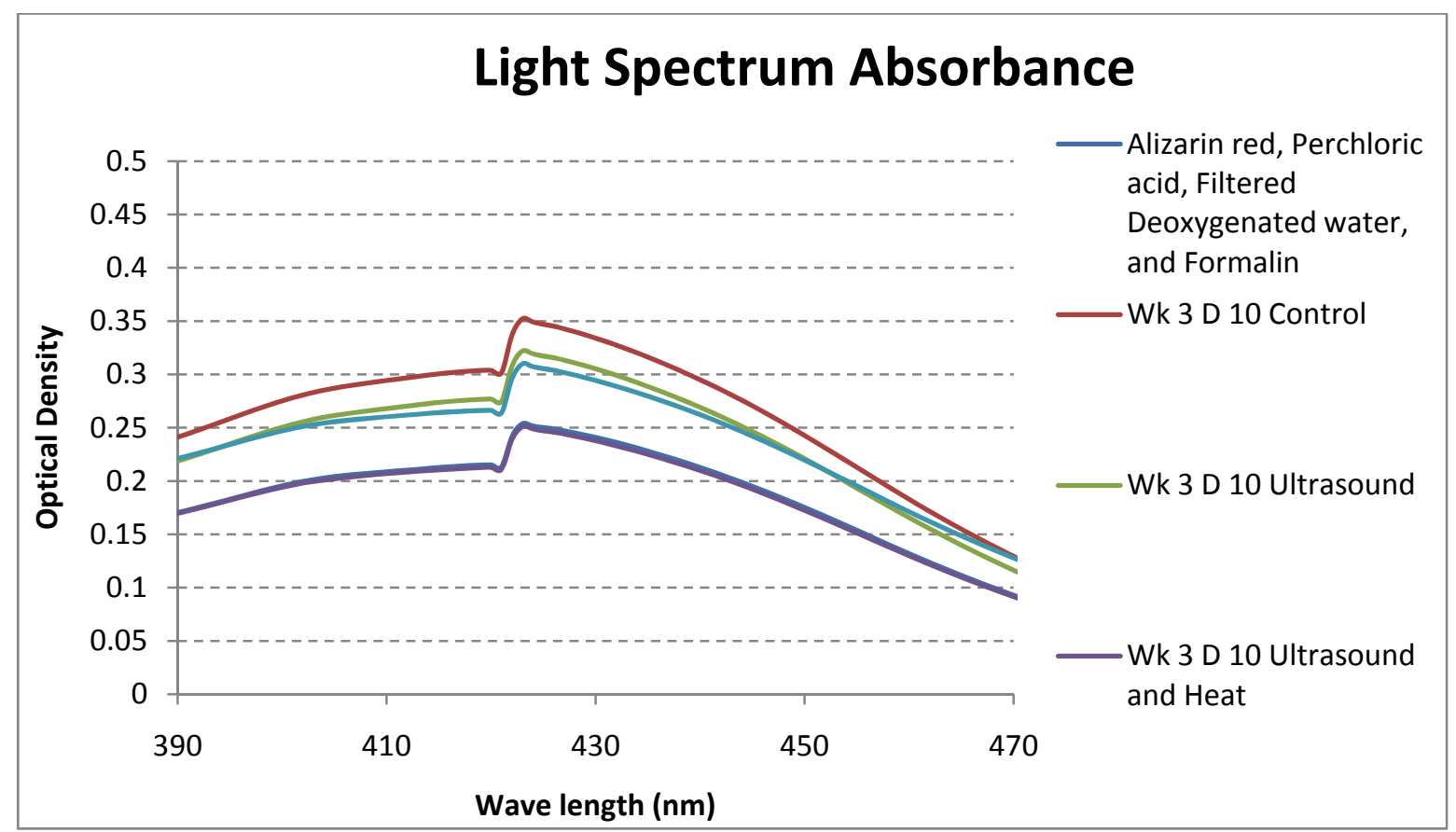

Figure 4-24: This graph is a magnification of Figure 4-23 between the wave lengths of 390 and $470 \mathrm{~nm}$. Spectrum analysis in range of $405 \mathrm{~nm}$ show that there are no rapid changes in absorption at this wave length. 


\section{Chapter 5 Discussion and Conclusion}

\subsection{Discussion}

Many adjuvant therapies have been tested with ultrasound; however the combination of low level heating and LIPUS has not been studied. The addition of heat to ultrasound is potentially a low cost and non-invasive technique to improve fracture healing. From practical point of view, combining the two therapies would be quite attractive since in biological tissues the ultrasound alone can be used as a non-invasive local heat source. This thesis studied the enhanced mineralization of bone cells as a model for fracture healing. The importance of the individual and combined therapies is that they reduce the time for fractures to heal and increase the functional properties of bone. Both early healing and improved bone function are associated with mineralization.

This study used the endpoint of mineralization as a method to gauge the effectiveness of the therapies. Staining for mineralization is a low cost and well tested method for assessing cells. Using this technique, the results of the experiment showed that there was a 4 fold increase for the 40 minute LIPUS (US40) treatment group when compared to the control. Based on published data, the result for the US40 was expected. Leung et al. (Leung et al 2004a) showed a 4 fold increase in mineralization after 4 weeks of ultrasound treatment when using human periosteal cells. The 40 minute heat (H40) treatment group also showed an expected increase of 1.2 fold in mineralization over the control. Shui et al (Shui and Scutt 2001), using an osteosarcoma derived cell line, showed an increase in mineralization of 1.25 fold when the cell cultures were heated to $39^{\circ} \mathrm{C}$ and 1.69 fold when the cell line were heated to $41^{\circ} \mathrm{C}$. An additive effect for the US40 + H40 group might be expected to be in the range of a 4.2 fold increase in 
mineralization. The US40 + H40 showed only a 4\% increase over the US40 treatment group. Due to the large variation of mineralization in the samples, this increase was not statistically significant. Therefore the outcome of our study shows no additive effect in the combined treatment group.

There are a couple of possibilities to explain why there was no additive effect found for the US40 + H40 treatment group. Three possible explanations are:

- The mechanisms of action of each treatment may have different onset timing.

- The mechanisms of action may not complement each other.

- The test method may not be sensitive enough to detect a difference between the treatment groups.

As described in chapter 1 , the mechanisms of action for ultrasound are thought to be the mechano-sensitization of cell integrins, the nano-motion of the cells or mode change of the ultrasound signal (mode change of the ultrasound signal is less significant for cell culture testing) (Pounder and Harrison 2008). Although the exact mechanisms are unknown, certain cellular level responses to ultrasound treatment have been shown to be repeatable. Increased mineralization is a distinct repeatable outcome from the application of ultrasound (Pounder and Harrison 2008). Multiple sequential cellular responses are essential precursors of mineralization. Precursors to mineralization have been shown to be ALP, MMP 13 and Osteocalsin among many others. According to Pounder et al. (Pounder and Harrison 2008) surface integrins mediate the mechanical signal on the cell surface and cause a cascade of changes throughout the cell. Integrins are a large family of cell adhesion molecules that mediate interactions between the extracellular environment and the cytoplasm (Milner and Campbell 2002). These integrins provide a physical link between the cytoskeleton and the extracellular matrix. According to 
Tang et al. (Tang et al 2006), these integrins are stimulated by the ultrasound signal from the surrounding matrix, and this stimulation causes the integrins to start a cascade of change in the cell causing a series of subsequent expressions eventually causing the cells to express calcium and the collagen matrix to mineralize. The combination of mechano-sensitive integrins and nano-scale motion cause by the ultrasound wave are theorized to be the mechanism behind ultrasound-cell interaction (Tang et al 2004, Tang et al 2006).

Although there are multiple examples of the temperature dependence of bone growth the mechanisms of action are even more elusive than ultrasound. Shui and Scutt (Shui and Scutt 2001) suggest that most likely the mechanism of action is related to the expression of Heat Shock Proteins (HSP); where HSP are molecular chaperones associated with cell survival after an insult. Shui suggests that HSP47 is involved with collagen synthesis and the expression of HSP47 is more likely to be induced in the presence of Transforming Growth Factor (TGF- $\beta 1$ ), where TGF- $\beta 1$ is released by the addition of heat. According to Naruse et al., LIPUS does not stimulate the expression of TGF- $\beta 1$ in MC3T3 cells (Naruse et al 2000). However, ultrasound does stimulate this growth factor in other cell lines or at higher intensities (Scheven et al 2007, Lu et al 2009). Calderwood and Asea (Calderwood and Asea 2002) suggests that when cells are exposed to temperatures over $40^{\circ} \mathrm{C}$ the production or Cyclo-oxygenase $2(\mathrm{COX}-2)$ and prostaglandin (PGE2) will increase. The mechanisms that are likely to cause improved calcification due to heat would not seem to have a negative synergistic interaction with ultrasound.

The combination of US40 + H40 concurrently may prove not to be additive. Although heat induces HSP and ultrasound induces mechano-sensitivity, both energy sources have a downstream effect of increasing COX-2 and PGE2. It is possible that these expressions are 
maximized with one energy source and cannot be expressed more with the addition of a second source.

When considering the time dependent expression of mineralization, previous studies have shown both heating and ultrasound need repeated treatments over several weeks to express differences in mineral staining verses a control (Unsworth et al 2007, Leung et al 2004a, Shui and Scutt 2001).

It is also possible that the additive effect of ultrasound and heating was missed simply because the testing was not appropriate. From Day 15 measurements, the standard error in light absorbance of the US40 and US40 + H40 treatment groups is 0.1 with an average absorbance of 0.6. H40 treatment produced an error 10 times smaller than either of US40 or US40 + H40. With an error of 0.01 and an average absorbance of approximately 0.2 , the error of both LIPUS groups is almost as large as the total absorbance of the H40 group.

Treatment results in all groups are studied only for a duration of 15 days after starting of the treatment. By Day 10, only 1/18 wells from the US40 group and 1/18 from the US40 + H40 group tested distinctly positive for mineralization. By day 15, 10/18 wells for the US40 group and 9/18 from the US40 + H40 wells tested distinctly positive for mineralization. From this assessment, it implies that the onset of mineralization occurred between days 10 and 15 . This may be a natural variation in time to mineralize, however it may also be a factor of the initial number of cells seeded in the well.

The process of seeding the wells was described in chapter 3. The total volume of cells was first mixed into media and then the combination of cells and media was seeded into the wells. Although, there should be the correct amount of cells in each well, it is likely that there was variation in the number of cells seeded in each well. As part of the differentiation process, 
cells will proliferate until they are confluent before the process of maturation will continue. The difference in this initial seeding may cause differences in the onset of mineralization and therefore slightly skew the results.

Distinct mineralization for cell cultures, treated with 20 minutes LIPUS at the same intensities used in this experiment, has been measured by other researchers between 10 and 25 days of treatment (Unsworth et al 2007, Pounder and Harrison 2008, Tang et al 2006). For heat treated cell cultures, distinct mineralization is measured around day 20 (Shui and Scutt 2001, Nørgaard et al 2006). For all published works, mineralization was used in conjunction with additional test methods to confirm increased mineralization.

\subsection{Conclusion}

It was shown in this thesis that the application of LIPUS through a polystyrene plate reduced the intensity significantly from $\mathrm{I}_{\mathrm{SATA}}(13 \mathrm{~mm})=24 \mathrm{~mW} / \mathrm{cm}^{2}$ with no polystyrene plate to $\mathrm{I}_{\mathrm{SATA}}(13 \mathrm{~mm})=10 \mathrm{~mW} / \mathrm{cm}^{2}$ when the polystyrene plate was introduced. Although, adjustments to the intensity were restricted with the device used, additional energy was deposited to the cell cultures through extended treatment times.

In conclusion, it was shown that the MC3T3-E1 bone cell cultures could significantly differentiate from pre-osteoblasts to osteoblasts by Day 5 in culture. The ability to differentiate all cell culture treatment groups was dependent on the addition of the differentiation supplement (DS) of ascorbic acid and $\beta$-glycerol phosphate. Cultures which were positive for the DS showed significant mineralization, while those that received no DS showed no mineralization throughout the culture time. 
Cells treated with 20 minutes/day of LIPUS (US20) showed statistically significant mineralization by day 10 . The mineralization was statistically significantly greater on days 10 and 15 with no statistical difference in mineralization of the US20 cultures on days 10 and 15.

Cells treated with 40 minutes/day of LIPUS (US40) or 40 minutes/day of LIPUS +40 minutes heat concurrently (US40 + H40), or 40 minutes/day of heat (H40), all showed statistically significant mineralization over the control, which received not treatment.

There was no statistically significant difference in mineralization between the US40 and the US40 + H40. It can be seen from the cumulative results that the onset of mineralization is between days 10 and 15.

When comparing the 20 minute LIPUS treatment to the 40 minute LIPUS treatment, the 40 minute treatment appeared to have much stronger mineralization that 20 minute group. The US40 treatment had optical absorbance of 0.16 on Day 10 and 0.61 on Day 15 . The US20 treatment had and optical absorbance of 0.17 on Day 10 and 0.13 on Day 15. The additional 20 minutes of LIPUS treatment seemed to increase mineralization most substantially between the day 10 and day 15 .

\subsection{Future work}

Refining the experimental protocol may provide an opportunity to reduce error in the experiment. Allowing the cells to remain in culture beyond 15 days may provide a method to reduce the effect of uneven seeding. As mentioned above, cells need to grow to confluence further stages of differentiation can progress. It may be possible that, if the cells are left for longer in culture, the mineral expressions may reach a steady state. The comparison of mineralization once the cultures have reached a steady state of mineralization may reduce the 
large errors (especially in the US40 and US40 + H40 treatment groups) so that subtle changes due to the addition of heat to LIPUS may become evident.

It addition, it may be possible that increasing the number of cells initially seeded may reduce the time needed for the culture to proliferate, therefore reducing the variation in initial time of proliferation.

The current experiment set up heat the water tank inside an incubator and holds the temperature through incubation heat, allowing a temperature variation of $\pm 0.5^{\circ} \mathrm{C}$. A thermostatic controlled water heater unit with a mixing pump to more evenly distribute the heat through the water may lead to better control on the heat and then a more controlled relationship between applied heat and mineralization. In addition, the relationship between heating effects in conjunction with ultrasound could be better understood.

As a standard, mineralization alone is not usually used as the only method to test treatment effectiveness for cells and cell cultures. To formalize the results in this thesis, an additional independent test method would be required. Colorimetric quantification has been a common method used to normalize cell culture results in many application of in vitro testing. Colourmetric quantification may account for seeding variations in cell culture wells. To set a base line for total number of cells in each culture a Tetrazolium Bromide Dye (MTT) could be used in this method (Davey and Lord 2003). The MTT assay is also an acceptable method for testing proliferation in cell cultures (Davey and Lord 2003). In this way, any cellular expressions will be normalized to the number of cells originally in the culture. In addition to the colorimetric measurements to assess cell counts, protein quantification is ideal method to assess earlier protein concentrations in cell cultures. In addition if cell viability is not required, protein concentration alone can be used to normalize protein or mineral content in cells or cell cultures. 
Either a Bicinchonic Acid Assay (BCA) (sensitive to temperature variations) or a Bradford assay (where the reference standard must be related to the target protein) for protein concentration could be used (Davey and Lord 2003). Both of these assays are commonly used and relatively sensitive. Alkaline Phosphatases (ALP) testing is a commonly used, specific test for the onset of differentiation with a 2-10 days time frame (Unsworth et al 2007, Shui and Scutt 2001, Nørgaard et al 2006). There are 2 methods to test for the ALP expression. A protein assay can be done based the cleavage of P-Nitro-Phenyl-Phosphate (pNPP) into P-Nitro-Phenol (pNP) cause by ALP (Leboy et al 1991). Additionally, ALP can be stained, rehydrated and measured with optical absorbance. However, this method is not as accurate as the protein assay (Shui and Scutt 2001). Further, ALP protein measurement can be made to mark and measure the specific onset of differentiation, and measure changes in the expressions of these proteins associated with changes in cell culture treatment. There are also possible artifacts of mineralization due to the addition of $\beta$-glycerol phosphate to be accounted for using protein quantification (Beresford et al 1993). Finally, Osteocalcin quantification is directly related to calcium levels in the culture and can be more easily normalized than calcium expression itself (Beck Jr. et al 2000).

A great deal of effort in this thesis has been put into the biological understanding of the effect of LIPUS and heating on cells. However, the LIPUS device used for these experiments was pre-packaged with intensity levels and pulse duration preset. Having the capability of further adjusting these levels along with heating levels could lead to more control in experiments and more effective results. 


\section{References}

Acevedo, P and Das-Gupta, D 2002 The measurement of the spatial average temporal average intensity Isata and ultrasonic power $\mathrm{W}$ in composite ultrasonic transducers for medical application Ultrasonics 40 1-8 819-21

Anderson, H C 2003 Matrix vesicles and calcification.Curr. Rheumatol. Rep. 53 222-6

Azuma, Ito, Harada, Takagi, Ohta and Jingushi 2001 Low-Intensity Pulsed Ultrasound Accelerates Rat Femoral Fracture Healing by Acting on the various Cellular Reactions in the Fracture Callus. pp 671-80

Bandow, K, Nishikawa, Y, Ohnishi, T, Kakimoto, K, Soejima, K, Iwabuchi, S, Kuroe, K and Matsuguchi, T 2007 Low-intensity pulsed ultrasound (LIPUS) induces RANKL, MCP-1, and MIP-1ß expression in osteoblasts through the angiotensin II type 1 receptor J. Cell. Physiol. 2112 392-8

Bartel, D L 2006 Orthopaedic Biomechanics: Mechanics and Design in Musculoskeletal Systems ed D T Davy and T M Keaveny (Upper Saddle River, N.J.: Pearson/Prentice Hall)

Beck Jr., G R, Zerler, B and Moran, E 2000 Phosphate is a specific signal for induction of osteopontin gene expression Proc. Natl. Acad. Sci. U. S. A. 9715 8352-7

Beresford, J N, Graves, S E and Smoothy, C A 1993 Formation of mineralized nodules by bone derived cells in vitro: A model of bone formation?Am. J. Med. Genet. 452 163-78

Bergen, G, Chen, L H, Warner, M and Fingerhut, L A 2008 Injury in the United States: 1995 Chartbook (Hyattsville, MD: National Center for Health Statistics)

Blair, R Cand Taylor, R A ed 2008 Biostatistics for the Health Sciences (Upper Saddle River, New Jersy, USA: Pearson Prentice Hall)

Bone and Joint Decade 2009 Measuring the Burden of Musculoskeletal Disease (Rosemont, IL: Bone and Joint Decade)

Bostrom, M P G, Yang, X and Koutras, I 2000 Biologics in bone healing Curr. Opin. Orthop. 115 40312

Buger, C 2008 Biological Nanostructured Materials, Mineralized Tissue (Stony Brook, NY USA: Chemistry Department , Stony Brook University)

Calderwood, S K and Asea, A 2002 Targeting HSP70-induced thermotolerance for design of thermal sensitizers Int. J. Hyperthermia 186 597-608

Canadian Institute for Health Information (CIHI) Injury Hospitalization by Nature of Injury 
Chang, W H -, Sun, J -, Chang, S - and Lin, J C 2002 Study of Thermal Effects of Ultrasound Stimulation on Fracture Healing Bioelectromagnetics 234 256-63

Claes, L and Willie, B 2007 The enhancement of bone regeneration by ultrasound Progress in Biophysics and Molecular Biology 93 1-3 384-98

Cobbold, R S C 2007 Foundations of Biomedical Ultrasound (Toronto: Oxford University Press)

Csányi, L J 1980 Reactions of molybdate ions with polyphenol reagents: Determination of molybdenum with Pyrocatechol Violet and Alizarin Red S Mikrochim. Acta 73 3-4 277-87

Davey, Jand Lord, M 2003 Essential Cell Biology: A Practical Approach ed J Davey and M Lord (Toronto: Oxford University Press)

Duarte, L R 1983 The Stimulation of Bone Growth by Ultrasound Archives of Orthopaedic and Traumatic Surgery 101 153-159

Dyson, M and Brookes, M 1983 Stimulation of bone repair by ultrasound.Ultrasound Med. Biol. Suppl 2 61-6

Erdogan, Ö and Esen, E 2009 Biological aspects and clinical importance of ultrasound therapy in bone healing J. Ultrasound Med. 286 765-76

Feril Jr., L B, Kondo, T, Zhao, Q - and Ogawa, R 2002 Enhancement of hyperthermia-induced apoptosis by non-thermal effects of ultrasound Cancer Lett. 178 163-70

Flour, M -, Ronot, X, Vincent, F, Benoit, B and Adolphe, M 1992 Differential temperature sensitivity of cultured cells from cartilaginous or bone origin Biol. Cell 751 83-7

Fox, S W, Chambers, T J and Chow, J W M 1996 Nitric oxide is an early mediator of the increase in bone formation by mechanical stimulation American Journal of Physiology - Endocrinology and Metabolism 2706 33-6

Franceschi, R T, Iyer, B S and Cui, Y 1994 Effects of ascorbic acid on collagen matrix formation and osteoblast differentiation in murine MC3T3-E1 cells J. Bone Miner. Res. 96 843-54

Gebauer, D, Mayr, E, Orthner, E and Ryaby, J P 2005 Low-intensity pulsed ultrasound: Effects on nonunions Ultrasound in Medicine \& Biology 3110 1391-402

Greenleaf, J F, Dutt, V, Muthupillai, R, Manduca, A and Ehman, R L 1996 Measurement of shear waves in tissue Proceedings of the 1996 18th Annual International Conference of the IEEE Engineering in Medicine and Biology Society. Part 4 (of 5) (Piscataway, NJ, United States: IEEE) pp 2172-3

Harle, J, Salih, V, Knowles, J C, Mayia, F and Olsen, I 2001 Effects of therapeutic ultrasound on osteoblast gene expression J. Mater. Sci. Mater. Med. 12 10-12 1001-4

Heckman, J, Ryaby, J, McCabe, J, Frey, J and Kilcoyne, R 1994 Acceleration of tibial fracture-healing by non-invasive, low-intensity pulsed ultrasound J. Bone Joint Surg. Am. 761 26-34 
Heybeli, N, Yeşildağ, A, Oyar, O, Gülsoy, U K, Tekinsoy, M A and Mumcu, E F 2002 Diagnostic ultrasound treatment increases the bone fracture-healing rate in an internally fixed rat femoral osteotomy model J. Ultrasound Med. 2112 1357-63

Hurrell, A 2007 Guidelines on Conducting Ultrasonic Intensity Measurements (Hampton Farm Business Park, Higher Bockhampton, Dorchester, Dorset DT2 8QH, UK: Precision Acoustics)

Kondo, T and Kano, E 1987 Enhancement of hyperthermic cell killing by non-thermal effect of ultrasound Int. J. Radiat. Biol. 511 157-66

Kristiansen, T K, Ryaby, J P, McCabe, J, Frey, J J and Roe, L R 1997 Accelerated healing of distal radial fractures with the use of specific, low-intensity ultrasound: A multicenter, prospective, randomized, double- blind, placebo-controlled study J. BONE JT. SURG. SER. A 797 961-73

Leboy, P S, Beresford, J N, Devlin, C and Owen, M E 1991 Dexamethasone induction of osteoblast mRNAs in rat marrow stromal cell cultures J. Cell. Physiol. 1463 370-8

Leon, S A, Asbell, S O, Arastu, H H, Edelstein, G, Packel, A J, Sheehan, S, Daskal, I, Guttmann, G G and Santos, I 1993 Effects of hyperthermia on bone. II. Heating of bone in vivo and stimulation of bone growth Int. J. Hyperthermia $9177-87$

Leskinen, J, Olkku, A, Lammi, M J, Mahonen, A and Hynynen, K 2009 Ultrasound induced activation of cell signaling on human MG-63 osteoblastic cells 8th International Symposium on Therapeutic Ultrasound pp 35-9

Leung, K S, Cheung, W H, Zhang, C, Lee, K M and Lo, H K 2004a Low Intensity Pulsed Ultrasound Stimulates Osteogenic Activity of Human Periosteal Cells Clin. Orthop. Relat. Res. 418 253-9

Leung, K, Lee, W, Tsui, H, Liu, P P and Cheung, W 2004b Complex Tibial Fracture Outcomes Following Treatment with Low-Intensity Pulsed Ultrasound : Pergamon Press.) pp 389-95

Liauh, C -, Shih, T -, Huang, H - and Lin, W - 2004 Reconstruction of the temperature field for inverse ultrasound hyperthermia calculations at a muscle/bone interface Med. Phys. 312 208-17

Lu, H, Qin, L, Lee, K, Cheung, W, Chan, K and Leung, K 2009 Identification of genes responsive to lowintensity pulsed ultrasound stimulations Biochem. Biophys. Res. Commun. 3783 569-73

Maes, J 2004 Time Dependant Failure Properties of Bovine Meniscal Attachments (Michigan USA: Michigan Technological University)

Mccormick, S M, Saini, V, Yazicioglu, Y, Demou, Z N and Royston, T J 2006 Interdependence of pulsed ultrasound and shear stress effects on cell morphology and gene expression Ann Biomed Eng 343 436-45

Milner, R and Campbell, I L 2002 The integrin family of cell adhesion molecules has multiple functions within the CNS J. Neurosci. Res. 693 286-91 
Naruse, K, Mikuni-Takagaki, Y, Azuma, Y, Ito, M, Ota, T, Kameyama, K - and Itoman, M 2000 Anabolic response of mouse bone-marrow-derived stromal cell clone ST2 cells to low-intensity pulsed ultrasound Biochem. Biophys. Res. Commun. 2681 216-20

Newton, I 1999 The Principia: Mathematical Principles of Natural Philosophy ed I B Cohen and A M Whitman (Berkeley: University of California Press)

Nørgaard, R, Kassem, M and Rattan, S I S 2006 Heat Shock-Induced Enhancement of Osteoblastic Differentiation of hTERT-Immortalized Mesenchymal Stem Cells pp 443-7

Ocheltree, K B and Frizzell, L A 1989 Sound field calculation for rectangular sources.IEEE Trans. Ultrason. Ferroelectr. Freq. Control 362 242-8

Pounder, N M and Harrison, A J 2008 Low intensity pulsed ultrasound for fracture healing: A review of the clinical evidence and the associated biological mechanism of action Ultrasonics 484 330-8

Precision Acoustics 2007 Precision Acoustics Hydrophone Manual (Dorchester, Dorcet: Precision Acoustics Ltd.)

Preston, R C ed 1991 Output Measurement for Medical Ultrasound (London ; New York: SpringerVerlag)

Puchtler, H, Meloan, S N and Terry, M S 1969 On the history and mechanism of alizarin and alizarin red S stains for calcium.J. Histochem. Cytochem. 172 110-24

Qin, L, Lu, H, Fok, P, Cheung, W, Zheng, Y, Lee, K and Leung, K 2006 Low-intensity pulsed ultrasound accelerates osteogenesis at bone-tendon healing junction Ultrasound in Medicine \& Biology 3212 1905-11

Reher, P, Harris, M, Whiteman, M, Hai, H K and Meghji, S 2002 Ultrasound stimulates nitric oxide and prostaglandin E2 production by human osteoblasts Bone 311 236-41

Ricardo, M 2006 The effect of ultrasound on the healing of muscle-pediculated bone graft in scaphoid non-union International Orthopaedics (SICOT) 302 123-7

Scheven, B A, Millard, J L, Cooper, P R, Lea, S C, Walmsley, A D and Smith, A J 2007 Short-Term In Vitro Effects of Low Frequency Ultrasound on Odontoblast-Like Cells Ultrasound Med. Biol. 339 1475-82

Schortinghuis, J, Stegenga, B, Raghoebar, G M and de Bont, L G 2003 Ultrasound stimulation of maxillofacial bone healing.Crit Rev Oral Biol Med 14 163-74

Scutt, A, Reading, L, Scutt, N and Still, K 2003 Bone Research Protocols ed M H Helfrich, S Ralston and I ebrary (Great Britain: Humana)

Selfridge, A 2009 Ultraound Data for Liquids and Plastics 
Shui, C and Scutt, A 2001 Mild heat shock induces proliferation, alkaline phosphatase activity, and mineralization in human bone marrow stromal cells and Mg-63 cells in vitro J. Bone Miner. Res. 164 731-41

Simon, A M, Manigrasso, M B and O'Connor, J P 2002 Cyclo-oxygenase 2 function is essential for bone fracture healing Journal of Bone and Mineral Research 176 963-76

Siska, P A, Gruen, G S and Pape, H C 2008 External adjuncts to enhance fracture healing: What is the role of ultrasound?Injury 3910 1095-105

Smith \& Nephew 2009

Physician's Instructions for use \& Package Insert. EXOGEN 4000+ ${ }^{\mathrm{TM}}$ Bone Healing System LowIntensity Ultrasound Bone Healing System for the Treatment of Nonunion and Fresh Fractures (Memphis TN: Smith \& Nephew) pp 44

Stanford, C M, Jacobson, P A, Eanes, E D, Lembke, L A and Midura, R J 1995 Rapidly forming apatitic mineral in an osteoblastic cell line (UMR 106-01 BSP) J. Biol. Chem. 27016 9420-8

Straub, S J, Johns, L D and Howard, S M 2008 Variability in effective radiating area at $1 \mathrm{MHz}$ affects ultrasound treatment intensity Phys. Ther. 88 150-7

Stroncek, J Dand Reichert, W M 2008 Overview of Wound Healing in Different Tissue Types (National Center for Biotechnology Information, National Library of Medicine, Building 38A, Bethesda, MD 20894: National Center for Biotechnology Information)

Sudo, H, Kodama, H, Amagai, Y, Yamamoto, S and Kasai, S 1983 In vitro differentiation and calcification in a new clonal osteogenic cell line derived from newborn mouse calvaria J. Cell Biol. 96 1 191-8

Tang, C -, Yang, R -, Huang, T -, Lu, D -, Chuang, W -, Huang, T - and Fu, W - 2006 Ultrasound stimulates cyclooxygenase-2 expression and increases bone formation through integrin, focal adhesion kinase, phosphatidylinositol 3-kinase, and Akt pathway in osteoblasts Mol. Pharmacol. 696 2047-57

Tang, C, Yang, R, Huang, T, Liu, S and Fu, W 2004 Enhancement of Fibronectin Fibrillogenesis and Bone Formation by Basic Fibroblast Growth Factor via Protein Kinase C-Dependent Pathway in Rat Osteoblasts Mol. Pharmacol. 663 440-9

ter Haar, G 2007 Therapeutic applications of ultrasound Prog. Biophys. Mol. Biol. 93 1-3 111-29

Trieb, K, Blahovec, H and Kubista, B 2007 Effects of hyperthermia on heat shock protein expression, alkaline phosphatase activity and proliferation in human osteosarcoma cells Cell Biochem. Funct. 256 669-72

Tsai, C L, Chang, W H and Liu, T K 1992 Preliminary studies of duration and intensity of ultrasonic treatments on fracture repair.Chin. J. Physiol. 35 121-6

Turner, C H, Takano, Y, Owan, I and Murrell, G A C 1996 Nitric oxide inhibitor L-NAME suppresses mechanically induced bone formation in rats American Journal of Physiology - Endocrinology and Metabolism 2704 33-4 
U.S. National Library of Medicine National Center for Biotechnology Information - PubChem Compound (Bethesda MD: National Center for Biotechnology Information , U.S. National Library of Medicine)

Unsworth, J, Kaneez, S, Harris, S, Ridgway, J, Fenwick, S, Chenery, D and Harrison, A 2007 Pulsed Low Intensity Ultrasound Enhances Mineralisation in Preosteoblast Cells Ultrasound Med. Biol. 339 1468-74

Wang, D, Christensen, K, Chawla, K, Xiao, G, Krebsbach, P H and Franceschi, R T 1999 Isolation and characterization of MC3T3-E1 preosteoblast subclones with distinct in vitro and in vivo differentiation/mineralization potential J. Bone Miner. Res. 146 893-903

Warden, S J, Bennell, K L, McMeeken, J M and Wark, J D 2000 Acceleration of Fresh Fracture Repair Using Sonic Accelerated Fracture Healing System (SAFHS): A Review Cacified Tissue International 66 157-163

Warden, S J, Fuchs, R K, Kessler, C K, Avin, K G, Cardinal, R E and Stewart, R L 2006 Ultrasound produced by a conventional therapeutic ultrasound unit accelerates fracture repair Phys. Ther. 868 $1118-27$

Waters, K R, Hughes, M S, Mobley, J and Miller, J G 2003 Differential forms of the Kramers-Krönig dispersion relations IEEE Trans. Ultrason. Ferroelectr. Freq. Control 501 68-76

Workman, J 1998 Applied Spectroscopy: A Compact Reference for Practitioners ed J Workman and A W Springsteen (San Diego: Academic Press)

Yang, R -, Lin, W -, Chen, Y -, Tang, C -, Huang, T -, Lu, B - and Fu, W - 2005 Regulation by ultrasound treatment on the integrin expression and differentiation of osteoblasts Bone 362 276-83

Zagzebski, J A 1996 Essentials of Ultrasound Physics (Toronto: Mosby)

Zemanek, J 1971 Beam Behavior Within the Nearfield of a Vibrating Piston J Acoust Soc Amer 491 pt 2 $181-91$ 